\title{
Angle-resolved photoemission spectroscopy (ARPES) studies of cuprate superconductors
}

by

Ari Deibert Palczewski

A dissertation submitted to the graduate faculty

in partial fulfillment of the requirements for the degree of

DOCTOR OF PHILOSOPHY

\author{
Major: Condensed Matter Physics \\ Program of Study Committee: \\ Adam Kaminski, Major Professor \\ David C. Johnston \\ John Lajoie \\ Patricia A. Thiel \\ Bruce Harmon
}

Iowa State University

Ames, Iowa

2010

Copyright (C) Ari Deibert Palczewski, 2010. All rights reserved. 


\section{DEDICATION}

I would like to dedicate this dissertation to my wife, Angela Haen, and my parents, Ammertte C. Deibert and Dennis W. Palczewski, for always telling me I could. 


\section{ACKNOWLEDGMENTS}

I would like to take this opportunity to express my thanks to all those who helped me with various aspects of my $\mathrm{PhD}$ research and graduate career.

First, I want to thank my advisor, Adam Kaminski, for his patience with my ever improving writing skills and his guidance, as we both worked through my education. One of the best scientists I know; his knowledge and experience were and are invaluable in my understanding of what it means to be a physicist.

Second, I thank my group members, Chang Liu and Takeshi Kondo. Chang thanks for accompanying me on multiple beamtimes, and correcting me on all the physics I thought I knew but actually did not. Takeshi thanks for writing the best analysis software ever and for helping me understand cuprates. In addition I would like to thank both for the countless hours of discussion on physics, culture, and politics.

I would also like to thank my committee members for their time and effort as I know they are extremely busy: , David C. Johnston, John Lajoie, Patricia A. Thiel, and Bruce Harmon (Dissertation defense substitute for Jörg Schmalian).

I would also like to acknowledge all the other professors and scientists at Ames Laboratory, Iowa State University, and at the multiple beam lines where I have worked; Alexei Fedorov - beam line 12 ALS Berkeley, CA, Eli Rotenberg and his staff - beam line 7 ALS Berkeley, CA, Wanli Yang - beam line 10 ALS; the staff at the Synchrotron Radiation Center Stoughton, Wisconsin; and Ming Shi, Markus Bendele, and Rustem Khasanov - X09LA-HRPES SLS, Paul Scherrer Institute, Switzerland.

I would additionally like to thank Todd Kempel for working through our classes together. Without his help I would never have passed my qualifiers. 
Additionally, I want to acknowledge all the other people who have helped me in and outside of the lab (in no particular order and with some people possibly accidentally omitted); Steven Binz, Steve Hahn, Stella Kim, Myron Hupalo, Yongbin Lee, Bert Crawley, Craig Ogilvie, Serge Bud'ko, Paul Canfield, Linda Shuck, Deb Schmidt, Larry Stoltenberg, Lori Hocket, Rebecca Shivvers, and Gloria Oberender.

Finally, I want to thank my wife, Angela, who never asked why I had to work late or go on trips for beamtime, only to tell her when I would return. 


\section{TABLE OF CONTENTS}

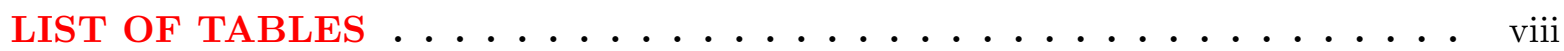

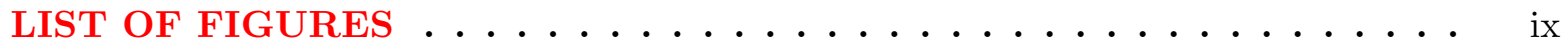

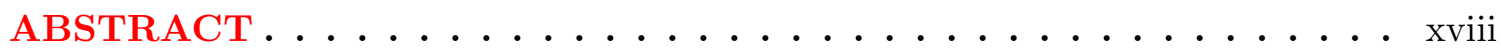

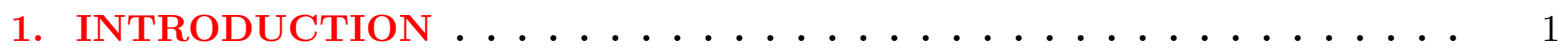

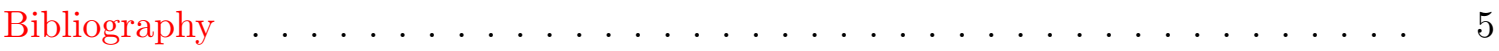

2. ANGLE-RESOLVED PHOTOEMISSION SPECTROSCOPY (ARPES):

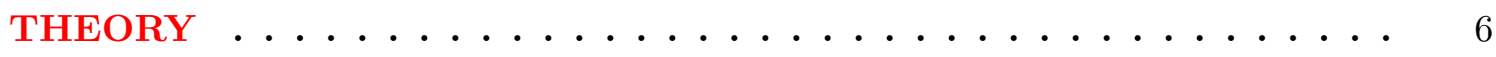

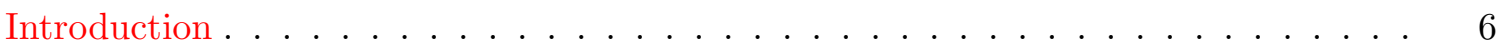

Photoelectron Kinetics . . . . . . . . . . . . . . . . . . 7

Three-Step Model . . . . . . . . . . . . . . . . . . . 9

Sudden Approximation . . . . . . . . . . . . . . . . . . . . . . 10

Self Energy and Spectral Function . . . . . . . . . . . . . . . . 12

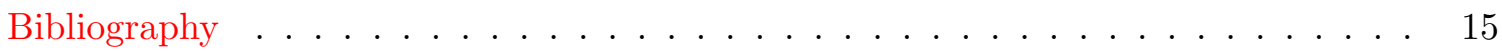

3. ANGLE-RESOLVED PHOTOEMISSION SPECTROSCOPY (ARPES):

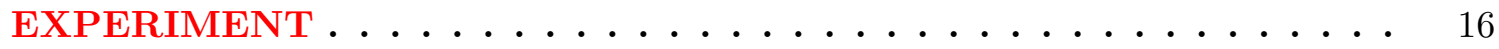

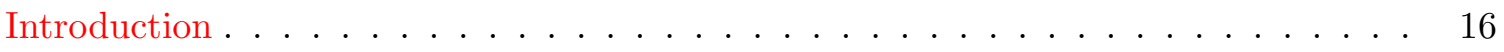

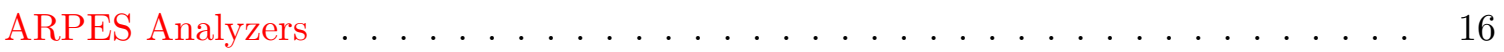

Electron lens . . . . . . . . . . . . . . . . . . . . . 17

Hemispherical analyzer . . . . . . . . . . . . . . . . . . 18

2-D MCP detector . . . . . . . . . . . . . . . . . . . . 19

Ultra High Vacuum . . . . . . . . . . . . . . . . . . 20 
Light Sources . . . . . . . . . . . . . . . . . . . . . 23

ARPES Data . . . . . . . . . . . . . . . . . . . . 27

Analyzing Data . . . . . . . . . . . . . . . . . . . . 28

Fermi level correction . . . . . . . . . . . . . . . . . . . . . 29

Normalization data . . . . . . . . . . . . . . . . . . . . . 29

Alignment of symmetry axis . . . . . . . . . . . . . . . . . . . 33

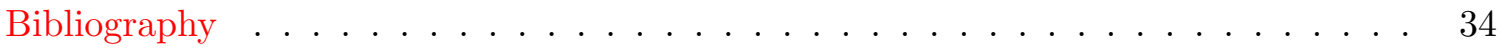

4. CUPRATE SUPERCONDUCTORS . . . . . . . . . . . 35

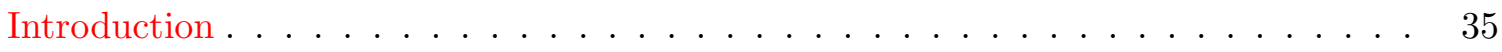

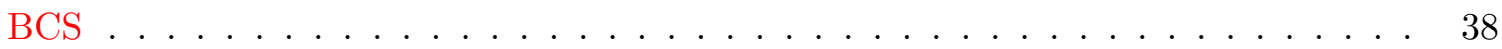

High Temperature Superconducting Cuprates . . . . . . . . . . . . . . . . . . 39

Crystal structure . . . . . . . . . . . . . . . . . . 39

Phase diagram . . . . . . . . . . . . . . . . . . . . . . 41

Cuprate Fermi surface . . . . . . . . . . . . . . . . . . 42

Fermi surface gaps . . . . . . . . . . . . . . . . . . . 45

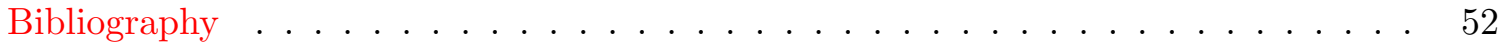

5. ORIGINS OF LARGE CRITICAL TEMPERATURE VARIATIONS IN

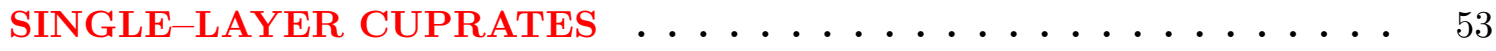

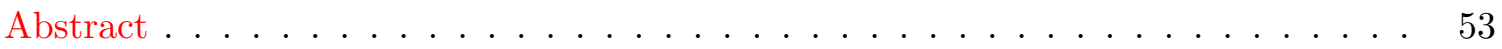

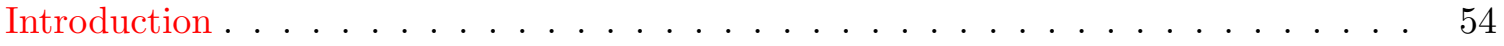

Experimental Details . . . . . . . . . . . . . . . . . . . 55

Data and Analysis . . . . . . . . . . . . . . . . . 56

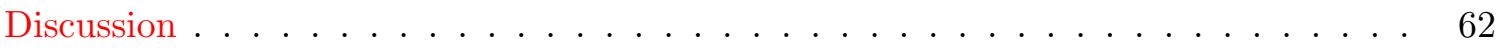

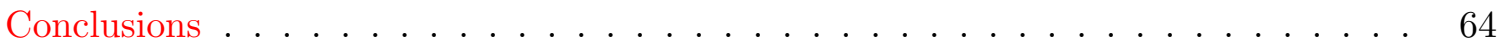

Acknowledgments . . . . . . . . . . . . . . . . . . . 64

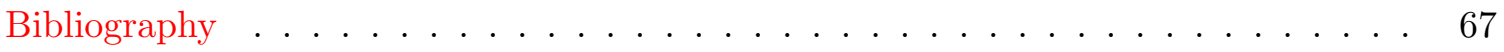


6. CONTROLLING THE CARRIER CONCENTRATION OF THE HIGHTEMPERATURE SUPERCONDUCTOR $\mathrm{Bi}_{2} \mathrm{Sr}_{2} \mathrm{CaCu}_{2} \mathrm{O}_{8+\delta}$ IN ANGLERESOLVED PHOTOEMISSION SPECTROSCOPY EXPERIMENTS • 68

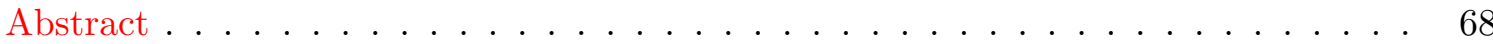

Introduction . . . . . . . . . . . . . . . . . . . . . . 69

Experimental Details . . . . . . . . . . . . . . . . . . . . 71

Increasing Carrier Concentration . . . . . . . . . . . . . . . . . . 73

Decreasing Carrier Concentration . . . . . . . . . . . . . . 76

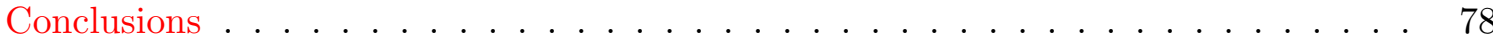

Acknowledgments . . . . . . . . . . . . . . . . . . . 80

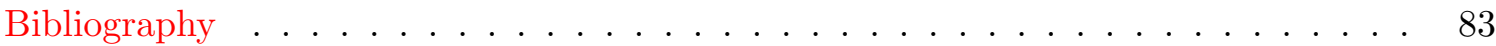

7. PROBING THE PARTICLE-HOLE SYMMETRY OF THE PSEUDOGAP IN HIGH TEMPERATURE SUPERCONDUCTING CUPRATES 84

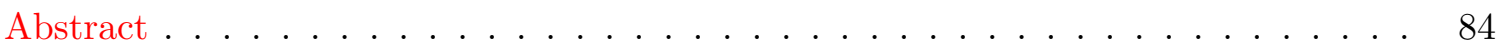

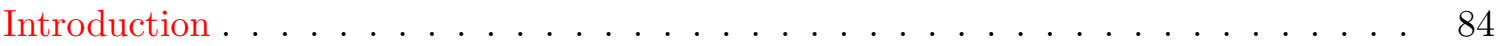

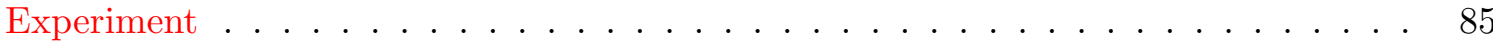

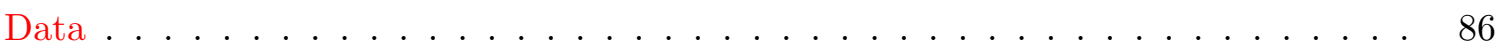

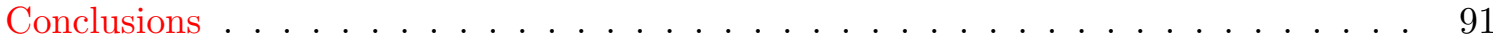

Acknowledgments . . . . . . . . . . . . . . . . . . . . . 92

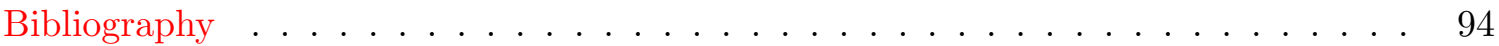

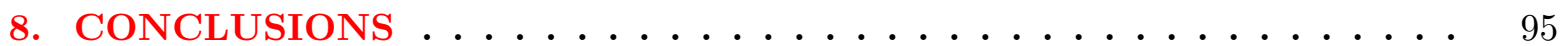




\section{LIST OF TABLES}

Table 5.1 Tight Binding fitting function $(\varepsilon(\vec{k}))$ and experimental fit for Bi2201, Tl2201 and Bi2212 [133] where $\varepsilon(\vec{k})=\sum c_{i} \eta_{i}(\vec{k})$ 


\section{LIST OF FIGURES}

Figure 2.1 Schematic diagram of a outgoing excited electrons in a ARPES setup.

Figure 2.2 Universal escape depth plot, i.e., mean free path of an electron in $\AA$ vs. the electron kinetic energy; showing the minimum mean free path occurs from 20-200 eV, the most frequently used energy range in modern ARPES experiments [35] . . . . . . . . . . . .

Figure 2.3 Angle-resolved photoemission spectroscopy: (a) the geometry of an ARPES experiment in which the emission direction of the photo-electron is specified by the polar $(\theta)$ and azimuthal $(\varphi)$ angles; (b) momentumresolved one-electron spectra for a non-interacting electron system with a single energy band dispersing across $E_{f}$ (The spectral function for the non-interacting case is simply a series of delta functions); and (c) the same spectra for an interacting Fermi-liquid system. For both noninteracting and interacting systems, the corresponding ground state ( $\mathrm{T}$ $=0 \mathrm{~K})$ momentum distribution function $\mathrm{n}(\mathrm{k})$ is also shown. Lower right in (c), photo-electron spectrum of gaseous hydrogen (solid line) and the ARPES spectrum of solid hydrogen (dashed line) developed from the gaseous one. Figure adapted from Damascelli et al. [32]. . . . . . . .

Figure 3.1 Schematic setup of a standard ARPES experiment. The protruding section is the electron lens, attached to the hemispherical energy analyzer. The acceptance angle, beam position, and distance from sample to lens vary with setup. The sample size is exaggerated. Diagram taken from D. S. Inosov $[50] . \ldots \ldots \ldots \ldots \ldots \ldots$ 
Figure 3.2 A schematic view of the paths of electrons as they move through the electron lens in a modern ARPES analyzer, each line represents the path of a photoelectron at a particular angle from the sample. Figure adapted from VG Scienta $[51] \ldots \ldots \ldots \ldots$

Figure 3.3 A schematic view of the path of the electrons as they move through the entrance slit of the electron lens to the 2-D MCP detector. When the electrons reach the MCP they are multiplied. The multiplied electron packets then excite a small area on a phosphorus screen releasing photons. The light from the phosphorus screen is then recoded by a charge coupled device (ccd) camera. The false color image represents the data collected by the camera. Figure adapted from beamline V-4 of SSRL website at Z.-X. Shen's laboratory $[52] \ldots \ldots$. . . . . . . . . .

Figure 3.4 Schematic diagram of a UHV setup, each box represents a different component of a complete UHV system and there location about the main chamber, each line represents a connection from one component to another. . . . . . . . . . . . . . . . .

Figure 3.5 ARPES system at Ames Laboratory: a) system during the bake-out procedure wrapped in multiple layers of tinfoil, heated by custom-built oven heater mounted on the bottom of the chamber; b) the same chamber in working condition after a bake-out. . . . . . . . . . . . .

Figure 3.6 Drawing of an off-the-shelf helium discharge lamp produced by VG Scienta model VUV5000. The energy output and bandwidths are listed to the right of the figure $[56] \ldots \ldots \ldots \ldots$

Figure 3.7 A schematic view of a modern synchrotron beam line from right to left, undulator, 4-jaw aperture, mirrors, plane grating (selects energy of the photons), slits and mirror, and finally the sample $[58] . \ldots . . .$. 
Figure 3.8 A 3-D plot of momentum-energy dispersion of Bi2212. The blue color indicates a higher intensity and black zero intensity moving through the planet earth color scale. This plot depicts different ways to slice though the 3-D matrix. The Fermi surface (FS) is the contour of high intensity at a constant energy cut at $\mathrm{E}_{F}$, the solid lines represent the fitted FS and the dashed line represent the shadow band. Cutting into/out of the page gives a 2-D image plot (e.g., the actual data recorded by the detector). Vertically cutting through the 2-D image plot creates a single energy distribution curve (EDC). Horizontally cutting through the 2-D image plot creates a momentum distribution curve (MDC). . . . . . .

Figure 3.9 Fermi surfaces of $\mathrm{EuRh}_{2} \mathrm{As}_{2}$ integrated within $40 \mathrm{meV}$ of $\mathrm{E}_{F}$ : a) taken with an incident photon energy of $105 \mathrm{eV}$; b) taken with an incident photon energy of $131 \mathrm{eV}$; and c) photon energy $/ k_{z}$ dependence taken at $k_{y}=0 \ldots \ldots \ldots \ldots \ldots \ldots \ldots \ldots \ldots \ldots \ldots \ldots \ldots \ldots \ldots \ldots \ldots \ldots$

Figure 3.10 Low statistics, high statistics, and high statistics normalized ARPES data, the bottom axis is in units of $\mathrm{k}$ or momentum/angle . . . . . .

Figure 3.11 Fermi function analysis obtained from polycrystalline gold: left - MCP channel location taken at three different angles (angle between the analyzer central axis and perpendicular to crystal surface) (-20 red, 20 green, and 50 blue); and right - Average $\mathrm{E}_{F}$ integrating each channel as a function of angle( the zero angle is defined when the sample plane is perpendicular to the analyzers lens central axis). . . . . . . . 
Figure 3.12 (a) EDC cut for a sample (black line Bi2212), gold (red line), and Fermi Function fit (Blue line) at 12K; (b) the same as (a) only zoomed in close to $\mathrm{E}_{f}$, the blue slashed triangle is the ghost region created by scanning mode; (c) 2-D image plot of Fermi divided Bi2212 close to $(\pi, 0)$ with the ghost spectrum left in at $140 \mathrm{~K}$; and (d) the same as (c) only with the ghost intensity removed. For the color scale the blue is the highest intensity, and black zero moving through the planet earth color scale. .

Figure 3.13 Fermi surface of OP90K Bi2212 measured at $12 \mathrm{~K}$ over two Brillouin zones with a $21.2 \mathrm{eV}$ helium excitation lamp. The top figure is the raw data measured by the analyzer. Each slice number represents a single angle bin; the actual angles were from -20 degrees to 50 degrees. The bottom figure is after rotating and scaling the data matrix to orientate the symmetry axises. . . . . . . . . . . . . . . . 32

Figure 4.1 Resistance vs. temperature for elemental mercury [75] . . . . . . . . 36

Figure 4.2 Transition temperature vs. discovery year for common superconduc-

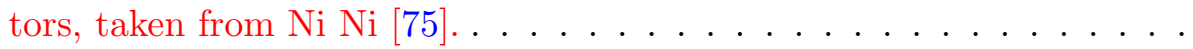

Figure 4.3 Idealized crystal structure of $\mathrm{Bi}_{2} \mathrm{Sr}_{2} \mathrm{CuO}_{6+\delta}, \mathrm{Bi}_{2} \mathrm{Sr}_{2} \mathrm{CaCu}_{2} \mathrm{O}_{8+\delta}$, and $\mathrm{Bi}_{2} \mathrm{Sr}_{2} \mathrm{Ca}_{2} \mathrm{Cu}_{2} \mathrm{O}_{10+\delta}$. The light brown sphere represents bismuth, the green sphere-strontium, the gray sphere-calcium, the blue sphere-copper, and the small red sphere-oxygen. The blue pyramids represent the bonding within the copper oxygen planes. The approximate maximum transition temperature of each optimally-doped compound is stated below each structure. Designed in VIC-II [78]. . . . . . . . . . . . . 40

Figure 4.4 Schematic doping phase diagram for both $\mathbf{n}$ (electron-doped) and $\mathbf{p}$ (hole-doped) cuprate superconductors. The doping range for selected materials are shown by the horizontal arrows $[82] \ldots . . . . . . .441$ 
Figure 4.5 Calculated band structure of optimally doped Bi2212: left - Fermi surface; and right - cuts along the FS showing the shape of the energy bands. . . . . . . . . . . . . . . . . . . .

Figure 4.6 FS of Bi2212 showing the main (red), umklapp (dashed blue), and shadow (dashed green) bands. . . . . . . . . . . . . . .

Figure 4.7 Top - Fermi surface map of optimally-doped Bi2212 taken in the second zone. In the color plot red corresponds to a high photoelectron count and blue to a lower photoelectron count, the red curve is the tight binding fit of the main band, and the yellow dotted curve is the tight binding fit of the shadow band. The upper right hand corner is a magnified view of the FS. Bottom - the size of the gap as a function of angle around the FS. . . . . . . . . . . . .

Figure 4.8 Pseudogap as a function of angle on the main band of UD75K Bi2212 at $\mathrm{T}=140 \mathrm{~K}$. Moving left to right: $\mathrm{EDC}$ at $k_{F}$ blue, symmetrized EDC $k_{F}$ red taken at $(\pi, 0)$; gap size as a function of angle(defined in far right figure); FS integrating within $\pm 10 \mathrm{meV}$ of $\mathrm{E}_{F}$, the black line from the middle figure show the portion of the FS that is not gapped, e.g., white, blue, and green FS (25-45 degrees), this region is called the Fermi Arc.

Figure 4.9 Left—pseudogap weight $\left(\mathrm{W}_{P G}\right)$ as a function of temperature on OD29K, the inserter shows a pictorial definition of the $\mathrm{W}_{P G}$. Right-coherent peak spectral weight $\left(\mathrm{W}_{C P}\right)$ as a function of temperature on OD29K, the inserter shows a pictorial definition of the $\mathrm{W}_{C P}$ adapted from Kondo et al. $[110] . \ldots \ldots \ldots \ldots \ldots \ldots \ldots$ 
Figure 4.10 Momentum dependence of the spectral weight as a percent of the total spectrum of Bi2201 at three different dopings; left - over-doped $T_{c}=29$ $\mathrm{K}$, middle - optimally-doped $T_{c}=35 \mathrm{~K}$, and right - under-doped $T_{c}=23$ $\mathrm{K}$. The red circles represent percent of the $\mathrm{W}_{P G}$ and the blue $\mathrm{W}_{C P}$. The vertical dotted line show the crossover angle between $\mathrm{W}_{P G}$ and $\mathrm{W}_{C P}$. Figure adapted from Kondo et al. [110]. . . . . . . . . . . . . . 47

Figure 5.1 (a) Schematic structure of $\mathrm{Bi}_{2} \mathrm{Sr}_{2} \mathrm{CuO}_{6+\delta}(\mathrm{Bi} 2201)$ and $\mathrm{Tl}_{2} \mathrm{Ba}_{2} \mathrm{CuO}_{6+\delta}$ (Tl2201). The small red dots represent oxygen atoms and the other larger atoms are labeled by the symbol on the left (Bi2201) or right (T12201). Each layer is made up of the particular atoms bonded to oxygen, with the double pyramids representing copper oxygen bonds. (b)-(c) SQUID magnetization curves for Bi2201 and Tl2201. . . . . . . 58

Figure 5.2 Intensity at the Fermi energy in multiple Brillouin zones for (a) Bi2201 and (b) Tl2201. All data was collected at a photon energy of $105 \mathrm{eV}$. High (low) intensity regions appear bright (dark) in the color map. (c) Tight binding fitting plots, Bi2212 [133] (black), Bi2201 (blue) and Tl2201 (red), fitting parameters for (c) are found in Table 1. . . . . .

Figure 5.3 Momentum distribution curve for (a)-(e) Bi2201 and (f)-(j) Tl2201 taken at $k_{y} / \pi=0.5,0.7,0.9,1.1$ and 1.3 . The lowest intensity corresponds to red while the highest intensity corresponds to dark blue moving through the color spectrum. The colored pictures are the original ARPES data while the black lines are tight binding fit. The tight binding fitting parameters for the black lines are located in Table 1, (k) FS taken from peak position of MDC for Bi2201 (blue dots) and Tl2201 (red crosses), (1) schematic MDC location for (a)-(j). . . . . . . . . . . 60

Figure 5.4 Intensity maps of Bi2201 taken around $(\pi, 0)$ for different carrier concentrations (a) $0.23 \%$, (b) $0.25 \%$, (c) $0.27 \%$, and (d) $0.29 \%$, with black line represents the tight binding fits for each doping level. . . . . . . . 61 
Figure 5.5 (a) Photon energy dependence for Tl2201 taken at around $(\pi, 0)$. Markers in (a) are at $49 \mathrm{eV}, 59 \mathrm{eV}$ and $74 \mathrm{eV}$ respectively, corresponding to the energy momentum cuts in (b), (c), and (d). In (b)-(d) dark blue corresponds to low intensity, while red corresponds to high intensity moving through the color spectrum. . . . . . . . . . .

Figure 6.1 ARPES spectrum of Bi2212 taken under poor vacuum conditions (CO and $\mathrm{CO}_{2}$ ): (a) sample EDC (energy distribution curves) taken at the antinode where the band crosses the Fermi energy at five different times (right after the cleave, 2, 10, 24, and 28 hours later ); (b) narrow view of (a); (c) time evolution of Bi2212's superconducting gap as a function of time; (d) the time evolution of Bi2212's superconducting gap under VUV photons (red) fitted with an exponential decay (blue), the temperature for (a)-(d) was set to $20 \mathrm{~K}$; and (e) C 1s, Sr $3 \mathrm{P}_{1} / 2$, Sr $3 \mathrm{p}_{3} / 2$ core level data from Bi2212 showing carbon deposits some time after cleaving and after cooling. . . . . . . . . . . . . . 72

Figure 6.2 (a) ARPES intensity map of freshly cleaved optimally-doped Bi2212 at $(\pi, 0)$, showing no bilayer splitting; (b) ARPES intensity maps on the same sample and the same location as in (a) only oxygen aged (in-situ overdoping) in a UHV system with a leak, showing bilayer splitting and a peak shift location of the Fermi momentum (the black line serves as a guide to the eye); (c) residual gas analyzer (RGA) spectrum after an optimal bake-out (red curve) and after a bake-out where there was a $10^{-10}$ Torr air leak (blue curve). . . . . . . . . . . . . . . . 74

Figure 6.3 (a)-(c) symmetrized ARPES EDC's for Bi2212 taken at three points near $(\pi, 0)$, showing the time evolution of the spectrum at $280 \mathrm{~K} . \quad \ldots 75$ 
Figure 6.4 (a) EDC at the Fermi momentum close to the anti-node before (green circles) and after (solid red squares) annealing at $280 \mathrm{~K}$ over 28 hours with their respective superconducting gaps $\Delta$; (b)-(c) intensities maps taken about the Fermi momentum close to $(\pi, 0)$ before and after an-

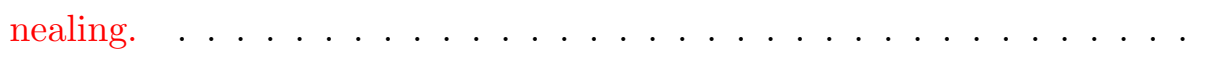

Figure 6.5 (a) $100 \mathrm{~K}$ symmetrized ARPES data taken at the Fermi momentum before and after annealing at $280 \mathrm{~K}$ for 28 hours; (b) ARPES intensities at $140 \mathrm{~K}$ before annealing; (c) ARPES intensities at $200 \mathrm{~K}$ after annealing. 78

Figure 6.6 Bi2212 EDC at the Fermi momentum close to $(\pi, 0)$ (a) just after cleaving at $100 \mathrm{~K}$ (red circles) and again after sitting at $100 \mathrm{~K}$ for 16 days (solid blue squares), (b) just after cleaving at $280 \mathrm{~K}$ (red circles) and again after sitting at $280 \mathrm{~K}$ for 8 days (solid blue squares). . . . . . .

Figure 6.7 ARPES intensity plots at the Fermi energy from the same sample at three consecutive times all at $12 \mathrm{~K}$ : (a) just after cleaving, (b) after a couple of days of VUV aging at low temperature, (c) after annealing at $280 \mathrm{~K}$ overnight; the upper right hand corner of (a)-(c) are the zoomed in images from the bottom left hand corner, the red and dotted yellow curves are from a tight binding fit for optimally doped Bi2212 as a guide to the eye, (d)-(f) the size of the superconducting gap as a function of angle $\phi$ from (a)-(c) respectively. . . . . . . . . . . . .

Figure 7.1 (a) FS map of Bi2212 UD75K at 140K, each circle represents the position of $k_{f}$ and each line represents the experimental cutting location in (b); (b) Fermi divided 2-D image plots taken at the 17 cutting locations in (a); (c) and (d) are the same as (a) and (b) only on Bi2201 UD20K. The width of each 2-D image plot in (b) and (d) is $\pm 0.1\left(\mathrm{k}-\mathrm{k}_{f}\right) / \pi$. For all plots black is zero intensity and blue is the most intense moving through the planet-earth color scale. . . . . . . . . . . . . 87 
Figure 7.2 Fermi divided energy distribution curves (EDC) from Figure 7.1. (a) Bi2212 UD75K taken at $140 \mathrm{~K}$ at the $17 k_{f}$ pointing in Figure 7.1 (a). (b) Bi2201 UD20K taken at $140 \mathrm{~K}$ at the $15 k_{f}$ points in Figure 7.1 (c). The thin curves, 1-7 in (a) and (b) are locations of a gapped band, and the bold curves, 8-17 in (a) and 8-15 in (b) are locations of a closed gap. 89

Figure 7.3 (a) Schematic location of the 4 cuts close to the end of the Fermi arc for (b)-(c). (b) Bi2212 OP90K taken at $110 \mathrm{~K}$ from the cutting locations in (a) taken in zone 1. (c) the same as (b) only on Bi2212 UD75K at 140 K. (d) the same as (c) only on Bi2201 UD20K in the second zone [reflected data (identical) from Figure 7.1 (d) data (6-9)]. The width of each 2-D image plot in (b) and (d) is $\pm 0.1\left(k-k_{f}\right) / \pi$, with the vertical line at $k_{f}$. For all plots black is zero intensity and blue is the most intense moving through the planet earth color scale. . . . . . . . . . 90 
xviii

\begin{abstract}
This dissertation is comprised of three different angle-resolved photoemission spectroscopy (ARPES) studies on cuprate superconductors. The first study compares the band structure from two different single layer cuprates $\mathrm{Tl}_{2} \mathrm{Ba}_{2} \mathrm{CuO}_{6+\delta}(\mathrm{Tl} 2201) \mathrm{T}_{c, \max } \approx 95 \mathrm{~K}$ and $\left(\mathrm{Bi}_{1.35} \mathrm{~Pb}_{0.85}\right)\left(\mathrm{Sr}_{1.47} \mathrm{La}_{0.38}\right) \mathrm{CuO}_{6+\delta}(\mathrm{Bi} 2201) \mathrm{T}_{c, \text { max }} \approx 35 \mathrm{~K}$. The aim of the study was to provide some insight into the reasons why single layer cuprate's maximum transition temperatures are so different. The study found two major differences in the band structure. First, the Fermi surface segments close to $(\pi, 0)$ are more parallel in Tl2201 than in Bi2201. Second, the shadow band usually related to crystal structure is only present in Bi2201, but absent in higher $\mathrm{T}_{c}$ Tl2201. The second study looks at the different ways of doping $\mathrm{Bi}_{2} \mathrm{Sr}_{2} \mathrm{CaCu}_{2} \mathrm{O}_{8+\delta}(\mathrm{Bi} 2212)$ in-situ by only changing the post bake-out vacuum conditions and temperature. The aim of the study is to systematically look into the generally overlooked experimental conditions that change the doping of a cleaved sample in ultra high vacuum (UHV) experiments. The study found two major experimental facts. First, in inadequate UHV conditions the carrier concentration of Bi2212 increases with time, due to the absorption of oxygen from $\mathrm{CO}_{2} / \mathrm{CO}$ molecules, prime contaminants present in UHV systems. Second, in a very clean UHV system at elevated temperatures (above about $200 \mathrm{~K}$ ), the carrier concentration decreases due to the loss of oxygen atoms from the Bi-O layer. The final study probed the particle-hole symmetry of the pseudogap phase in high temperature superconducting cuprates by looking at the thermally excited bands above the Fermi level. The data showed a particle-hole symmetric pseudogap which symmetrically closes away from the nested FS before the node. The data is consistent with a charge density wave (CDW) origin of the pseudogap, similar to STM checkerboard patterns in the pseudogap state.
\end{abstract}




\section{CHAPTER 1. INTRODUCTION}

It has been 24 years since the discovery of high temperature superconductivity in cuprates [1]. Although, we have learned a lot about their fascinating physics, many questions, including the most important one - "What is the mechanism of pairing in these materials?" - remains unsolved. Unlike conventional superconductors, such as lead or mercury, whose superconductivity can be fully explained within theory proposed by Bardeen-Cooper-Schrieffer (BCS) theory [2-4]. In cuprates, strong electronic correlations lead to complicated physics beyond the framework of conventional theory.

The undoped parent compounds are characterized by an antiferromagnetic insulating ground state, where conventional band structure calculations predict a conducting material. In this so-called Mott insulator state, strong Coulomb repulsion leads to localization of electrons on $\mathrm{Cu}$ sites and makes the material an insulator. Cuprates strong electronic correlations lead to complicated physics beyond the framework of conventional theory. Upon doping of holes or electrons, the crystal's antiferromagnetism is suppressed and the materials enter a strange, so-called pseudogap state at low temperatures - whose origins are still highly disputed [5]. After further doping, the samples become superconducting at zero temperature and finally just metallic.

Many scientific tools have been used to probe various aspects of cuprates and their superconductivity. One tool, Angle-Resolved Photoemission Spectroscopy (ARPES), experienced rapid development during the "cuprate era." ARPES is the only technique that can map the full four-dimensional $\left(k_{x}, k_{y}, k_{z}\right.$, and energy) band structure of a material. ARPES has revealed a number of fascinating phenomena in cuprates, such as the direct observation of the

superconducting gap [6] and its anisotropy [7,8], confirmation of the $d$-wave symmetry of the 
order parameter, direct observation of the pseudogap and its anisotropy [9-11], nodal quasiparticles [12], renormalization effects [13-15], and many others [16, 17]. Although we know a lot about cuprates, many questions remain: "What is the origin of pairing?"; "What is the origin of the pseudogap?"; "Does the pseudogap compete with superconductivity?"'; "Why does $\mathrm{T}_{c}$ scale with the number of layers?"; among others. This dissertation attempts to answer some of these questions by studying the electronic structure of cuprates with ARPES.

Chapter 2 is an overview of the different mathematical theories used to describe ARPES and its methodologies. Chapter 3 presents experimental methods and analysis used in modern ARPES experiments, beginning with the analyzer and ultrahigh vacuum environment, and ending with data analysis. Chapter 4 introduces cuprate superconductors, and their electronic structure.

Chapters 5, 6, and 7 are three studies looking in the electronic structure of cuprates by means of ARPES. Chapter 5 compares the Fermi surfaces of single layered cuprates $\mathrm{Tl}_{2} \mathrm{Ba}_{2} \mathrm{CuO}_{6+\delta}(\mathrm{Tl2201})$ and $\left(\mathrm{Bi}_{1.35} \mathrm{~Pb}_{0.85}\right)\left(\mathrm{Sr}_{1.47} \mathrm{La}_{0.38}\right) \mathrm{CuO}_{6+\delta}(\mathrm{Bi} 2201)$. The main focus of the study was to try and explain why Tl2201 has a superconducting transition temperature $\left(\mathrm{T}_{c, \max }\right)$ close to $95 \mathrm{~K}$ while Bi2201's $\mathrm{T}_{c, \max } \sim 35 \mathrm{~K}$; by comparing the shape and features of the Fermi surface (FS). Chapter 5 is published in Physical Review B: 78, 054523 (2008). Chapter 6 studies the different ways to dope $\mathrm{Bi}_{2} \mathrm{Sr}_{2} \mathrm{CaCu}_{2} \mathrm{O}_{8+\delta}$ (Bi2212) using only vacuum conditions and temperature, carefully examining the causes of in-site doping. Although these affects are common in ARPES measurements no one has published a careful study of these effects until this work. Chapter 6 is published in Physical Review B: 81, 104521 (2010). Chapter 7 probes the symmetry of the pseudogap state in Bi2212 and Bi2201. One of the most fervent debates in the world of cuprates is the origins and symmetry of the pseudogap state. There are multiple theoretical [18] and experimental [19-21] studies suggesting a shift in the pseudogap. This is contradictory to others who suggest a symmetric gap [22-25]. We probed the symmetry of the pseudogap by looking at the thermal excitation of states above the Fermi level to ascertain the correct symmetry. Chapter 7 is in preparation for submission to Physical Review Letters. Finally Chapter 8 summarizes the main conclusions from Chapters 5, 6, and 7, including the 
contributions each chapter has made to the field of cuprates.

\section{Bibliography}

[1] J. G. Bednorz and K.A. Muller, Z. Phys. B 64, 189 (1986).

[2] L. N. Cooper, Phys. Rev 104, 1189 (1956).

[3] J. Bardeen, L. N. Cooper, and J. R. Schrieffer, Phys. Rev. 106, 162 (1957).

[4] J. Bardeen, L. N. Cooper, and J. R. Schrieffer, Phys. Rev. 108, 1175 (1957).

[5] M. R. Norman, Science 325, 1080 (2010).

[6] C. G. Olson, R. Liu, A. -B. Yang, D. W. Lynch, A. J. Arko, R. S. List, B. W. Veal, Y. C. Chang, P. Z. Jiang, and A. P. Paulikas, Science 245, 731 (1989).

[7] Z.-X. Shen, D. S. Dessau, B. O. Wells, D. M. King, W. E. Spicer, A. J. Arko, D. S. Marshall, L. W. Lombardo, A. Kapitulnik, P. Dickinson, S. Doniach, J. Dicarlo, T. Loeser, and C. H. Park, Phys. Rev. Lett. 70, 1553 (1993).

[8] H. Ding, M. R. Norman, J. C. Campuzano, M. Randeria, A. F. Bellman, T. Yokoya, T. Takahashi, T. Mochiku, and K. Kadowaki, Phys. Rev. B 54, R9678 (1996).

[9] H. Ding, T. Yokoya, J. C. Campuzano, T. Takahashi, M. Randeria, M. R. Norman, T. Mochiku, K. Kadowaki, and J. Giapintzakis, Nature (London) 382, 51 (1996).

[10] A. G. Loeser, Z.-X. Shen, D. S. Dessau, D. S. Marshall, C. H. Park, P. Fournier, and A. Kapitulnik, Science 273, 325 (1996).

[11] M. R. Norman, H. Ding, M. Randeria, J. C. Campuzano, T. Yokoya, T. Takeuchi, T. Takahashi, T. Mochiku, K. Kadowaki, P. Guptasarma, and D. G. Hinks, Nature (London) 392, 157 (1998).

[12] A. Kaminski, J. Mesot, H. Fretwell, J. C. Campuzano, M. R. Norman, M. Randeria, H. Ding, T. Sato, T. Takahashi, T. Mochiku, K. Kadowaki, and H. Hoechst, Phys. Rev. Lett. 84, 1788 (2000). 
[13] T. Valla, T. E. Kidd, W.-G. Yin, G. D. Gu, P. D. Johnson, Z.-H. Pan, and A. V. Fedorov, Phys. Rev. Lett. 98, 167003 (2007).

[14] P. V. Bogdanov, A. Lanzara, S. A. Kellar, X. J. Zhou, E. D. Lu, W. J. Zheng, G. Gu, J.-I. Shimoyama, K. Kishio, H. Ikeda, R. Yoshizaki, Z. Hussain, and Z.-X. Shen, Phys. Rev. Lett. 85, 2581 (2000).

[15] A. Kaminski, M. Randeria, J. C. Campuzano, M. R. Norman, H. Fretwell, J. Mesot, T. Sato, T. Takahashi, and K. Kadowaki, Phys. Rev. Lett. 86, 1070 (2001).

[16] A. Damascelli, Z. Hussain, and Z.-X. Shen, Rev. Mod. Phys. 75, 473 (2003).

[17] J. C. Campuzano, M. R. Norman, and M. Randeria, in The Physics of Superconductors, Vol. 2, ed. K. H. Bennemann and J. B. Ketterson, (Springer, Berlin), p. 167 (2004).

[18] M. R. Norman, D. Pines, and C. Kallin, Adv. Phys. 54, 715 (2005).

[19] H.-B. Yang, J. D. Rameau, P. D. Johnson, T. Valla, A. Tsvelik, and G. D. Gu, Nature 456, 77 (2008).

[20] H.-B. Yang, J. D. Ramaeu, Z.-H. Pan, G.D. Gu, P. D. Johnson, R. H. Claus, D. G. Hinks, and T. E. Kidd, arXiv:1008.3121v1 (2010).

[21] M. Shi, A. Bendounan, E. Razzoli, S. Rosenkranz, M. R. Norman, J. C. Campuzano, J. Chang, M. Mnsson, Y. Sassa, T. Claesson, O. Tjernberg, L. Patthey, N. Momono, M. Oda, M. Ido, S. Guerrero, C. Mudry, and J. Mesot, EPL 88, 27008 (2009).

[22] W. S. Lee, I. M. Vishik, K. Tanaka, D. H. Lu, T. Sasagawa, N. Nagaosa, T. P. Devereaux, Z. Hussain, and Z.-X. Shen, Nature 450, 81 (2007).

[23] A. Kanigel, U. Chatterjee, M. Randeria, M. R. Norman, G. Koren, K. Kadowaki, and J. C. Campuzano, Phys. Rev. Lett. 101, 137002 (2008).

[24] T. Sato, T. Kamiyama, T. Takahashi, J. Mesot, A. Kaminski, J. C. Campuzano, H. M. Fretwell, T. Takeuchi, H. Ding, I. Chong, T. Terashima, and M. Takano, Phys. Rev. B 64, 054502 (2001). 
[25] T. Takahashi, T. Sato, H Matsui, and K. Terashima, New J. Phys. 7, 105 (2005). 


\section{CHAPTER 2. ANGLE-RESOLVED PHOTOEMISSION SPECTROSCOPY (ARPES): THEORY}

\section{Introduction}

The photoelectric effect was first observed by Hertz [26] where ultraviolet light caused a current in a solid and later was explained with the quantum mechanical nature of light by Einstein [27]. Einstein recognized an energetic photon can be absorbed by an electron in a solid. These photo-electrons are emitted from the solid with a maximum kinetic energy:

$$
E_{k i n}=h \nu-\phi
$$

where $\nu$ is the photon frequency, $h$ is Plank's constant $\left(4.135 \times 10^{-15} \mathrm{eV} \cdot \mathrm{s}\right)$, and $\phi$ is the work function (the minimum energy required to remove the electron from the solid). This chapter will review the photoelectric effect in the context of angle-resolved photoemission spectroscopy (ARPES), in particular, how one can use it to explain the experimentally obtained energy-momentum dispersion relationship in solids. This chapter is divided into four different sections: 1) photoelectron kinetics (single photon-electron classical interactions); 2) three-step model (basic quantum mechanical approach to an electron interaction with an electro-magnetic field); 3) sudden approximation (perturbative approximation made in ARPES); and 4) spectral function (many-body interactions in solids, probed by ARPES). The chapter is adopted from the following two sources, unless otherwise noted: Lynch and Olson's Photoemission Studies of High-Temperature Superconductivity [28] and Hufner's Photoelectric Spectroscopy [29]. 


\section{Photoelectron Kinetics}

When a beam of monochromatic photons with an energy greater than the work function $(\phi)$ interacts with a sample's electrons, the electrons can absorb the photons and gain sufficient energy to escape from the sample. By measuring the energy and momentum of the photoelectron outside the sample and using conservation laws, one can derive the properties of the electron prior to the photoemission process. This information can then be used to reconstruct the energy-momentum dispersion relationship (band structure), and many other electronic properties of the solid. The momentum of the photo-electron in the vacuum $(K)$ is related to the energy of the photo-electron, $E_{k i n}$, by the following equation:

$$
K=\frac{\sqrt{2 m E_{k i n}}}{\hbar}
$$

where $\mathrm{m}$ is the mass of the free electron. The momentum of the electron can be written as the sum of two components, parallel and perpendicular to the sample's surface:

$$
\overrightarrow{K_{\|}}=\vec{K}_{x}+\vec{K}_{y}
$$

and

$$
\overrightarrow{K_{\perp}}=\overrightarrow{K_{z}}
$$

The momentum and energy relation for the individual components, according to their emission angles in spherical coordinates are as follows:

$$
\begin{aligned}
& K_{x}=\frac{\sqrt{2 m E_{k i n}} \sin \theta \cos \varphi}{\hbar}, \\
& K_{y}=\frac{\sqrt{2 m E_{k i n}} \sin \theta \sin \varphi}{\hbar},
\end{aligned}
$$

and

$$
K_{z}=\frac{\sqrt{2 m E_{k i n}} \cos \theta}{\hbar} .
$$

The two angles are defined in Figure 2.1 (a). These are the energy-momentum $\left[E_{k i n}(K)\right]$ relationships for the electron outside the sample, however, one actually wants to determine $\mathrm{E}(k)$, the energy-momentum relation inside the sample. Lower case " $k$ " is used for the momentum of the electron in the sample, and a upper case " $K$ " for the momentum of the vacuum electron. 


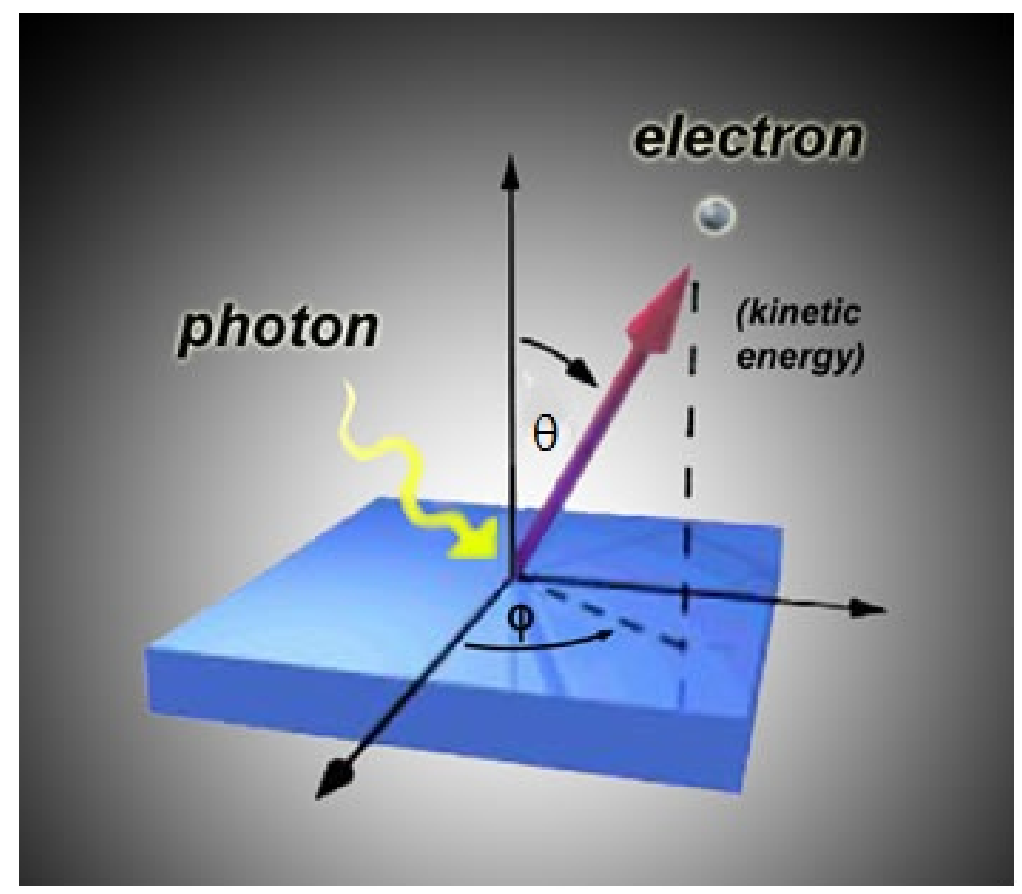

Figure 2.1 Schematic diagram of a outgoing excited electrons in a ARPES setup.

During the photoemission process, some of the components of the electrons momentum and energy are conserved while others are not. Because of the translational symmetry in the $\mathrm{x}-\mathrm{y}$ plane (the surface plane of the sample) and the absents of a potential in the plane, the electron's parallel component of momentum is conserved:

$$
k_{\|}=K_{\|}=\left(\frac{2 m}{\hbar^{2}} E_{k i n}\right)^{\frac{1}{2}} \sin (\theta)=\left(\frac{2 m}{\hbar^{2}}\left[E_{b i n d}+h \nu-\phi\right]\right)^{\frac{1}{2}} \sin (\theta)
$$

The momentum of the photon is very small compared to the momentum of the photoelectrons (for the typical photon energies used in ARPES experiments) so it was neglected. The situation $k_{\perp}$ becomes more complicated because of the presence of a surface potential $\left(V_{o}\right)$ whose gradient is perpendicular to the surface. The surface potential arises from the zero energy difference between the crystal and the vacuum. The perpendicular component of momentum can be written in terms of the unknown surface potential, $V_{o}$.

$$
k_{\perp}=\left(\frac{2 m}{\hbar^{2}}\left(E_{k i n}+V_{o}\right)\right)^{\frac{1}{2}} \cos (\theta) .
$$


Knowing the value for $\mathrm{k}_{\perp}$ is generally impossible without making some assumptions because $V_{o}$ is unknown, but many materials including cuprates which are inherently 2-D (no electron coupling between crystallographic planes), $k_{\perp}$ is zero and can be ignored from our consideration. There are experimental ways to determine $V_{o}$ from the periodicity of the Fermi surface in 3-D, this will be discussed in Chapter 3 .

\section{Three-Step Model}

The previous section was a semiclassical approach to photoelectron kinetics. However, a more formal quantum mechanical description is needed to understand the intricacies of ARPES. The total photoemission intensity, coming from the optical excitation of electrons in a 2-D material, is proportional to the transition probability of an electron being optically excited from the ground state $\Psi_{i}^{N}$ to a possible final state $\Psi_{f}^{N}$.

$$
I(E, K, h \nu) \propto \sum_{i, f}^{N} \frac{2 \pi}{\hbar}\left|\left\langle\Psi_{f}^{N}\left|H_{\mathrm{int}}\right| \Psi_{i}^{N}\right\rangle\right|^{2} \times \delta\left(E_{f}^{N}-E_{i}^{N}-h \nu\right) \times \delta\left(k_{i}-\mathbf{G}-K\right)
$$

where

$$
\left\langle\Psi_{f}^{N}\left|H_{\text {int }}\right| \Psi_{i}^{N}\right\rangle
$$

is the transition probability (proportional to Fermi's golden rule) and $\mathbf{G}$ is a reciprocal lattice

vector given by the Fourier transform of the real space lattice periodicity, $E_{i}^{N}=E_{i}^{N-1}-E_{B}^{k}$ are the initial energies for a system of $N$ electrons and $E_{f}^{N}=E_{f}^{N-1}+E_{k i n}$ is the final state. The interaction is treated as a perturbation in the system where the perturbing Hamiltonian is

$$
H_{\mathrm{int}}=\frac{e}{2 m c}(A \bullet p+p \bullet A)=\frac{e}{2 m c}(A \bullet p),
$$

where $\mathbf{A}$ in the electromagnetic vector potential and $\mathbf{p}$ is the electronic momentum operator. This Hamiltonian comes about by replacing the momentum operator $\mathbf{p}$ by $[\mathbf{p}-(\mathrm{e} / \mathrm{c}) \mathbf{A}]$, a system of electrons in an electromagnetic field. The Hamiltonian is then reduced by assuming the electrons are in the linear optical region. This assumption drops the $\mathbf{A}^{2}$ part of the Hamiltonian 
because the field is small compared to the momentum of the electron in the energy range (10$200 \mathrm{eV})$.

Because of the complexity of the photoemission process the resulting photoemission intensity can be thought of in three independent steps [30-32]. Step 1: optical excitation of the electron in the bulk, a photon interacts with a single electron. The probability of transition comes from Eq. (2.11) where the electron interacts with the electromagnetic potential $\mathbf{A}$ in-

side the crystal and the energy and momentum are conserved, e.g., $\delta\left(E_{f}^{N}-E_{i}^{N}-h \nu\right)$ and $\delta\left(k_{i}-\mathbf{G}-K\right)$. Step 2: travel of the electron to the surface, the electron travels to the surface of the crystal with a probability proportional to its mean free path. If the electron interacts in-elastically with the system during this stage, it is considered to be a secondary electron that will add to the high energy background of the system. This mean free path is generally approximated to be close to $3 \AA$ for a material like cuprates [33, 34]. The mean free path can also be approximated by the escape depth of simple metals [36]. The classical example of escape depth vs. photon energy is given by Figure 2.2. Sixty-six percent or $(1-1 / e) \%$, where e $=2.71828$, of the electrons at the escape depth reach the surface of the crystal without scattering. Step 3: escape of the photo-electron into the vacuum, the electron is only allowed to leave the surface if it has enough energy as compared to the work function of the material. Specifically, the perpendicular component of momentum needs to be larger than the work function and the binding energy, e.g.,

$$
\frac{\hbar^{2} k_{\perp}^{2}}{2 m} \geq\left|E_{o}\right|+\phi
$$

\section{Sudden Approximation}

While the three-step model works as a conceptual idea, there are some issues that still need addressed in Step 1. The problem that complicates the situation is once the system is perturbed, the system changes from an $N$ electron state to a system of $N-1$ electrons. This system will then relax, changing A. This is usually treated within an assumption known as the sudden approximation. In this limit, there is no interaction between the post collision 


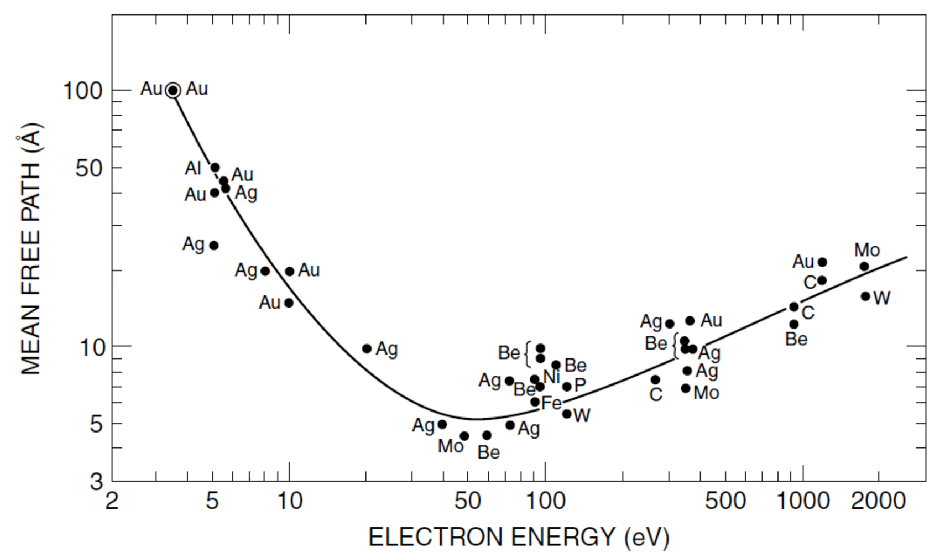

Figure 2.2 Universal escape depth plot, i.e., mean free path of an electron in $\AA$ vs. the electron kinetic energy; showing the minimum mean free path occurs from $20-200 \mathrm{eV}$, the most frequently used energy range in modern ARPES experiments [35].

photo-electron and the system left behind (i.e., the electron is instantaneously removed from the system and the reaction is instantaneous and discontinuous at the time of interaction). The limit may be invalid at lower photon energy, where the relaxation time approaches the escape time. However, it has been shown for cuprates even at low energies, this is less important and will be ignored for this dissertation [37].

If the photoemission process is assumed to be instantaneous, the initial and final states in a system of $N$ electrons can be broken into two states,

$$
\Psi_{f}^{N}=\mathrm{A} \phi_{f}^{k} \Psi_{f}^{N-1}
$$

where $\mathrm{A}$ is the antisymmetric operator which properly antisymmetrizes the N-electron wavefunction so the Pauli principle is satisfied. $\phi_{f}^{k}$ is the photo-electron's wave-function and $\Psi_{f}^{N-1}$ is the final state wave-function of the system left behind. The matrix element or square of the transition probability can be written as follows:

$$
w_{f, i}=\frac{2 \pi}{\hbar}\left|\left\langle\Psi_{f}^{N}\left|H_{\mathrm{int}}\right| \Psi_{i}^{N}\right\rangle\right|^{2} \propto\left|\left\langle\phi_{f}^{k}\left|H_{\mathrm{int}}\right| \phi_{i}^{k}\right\rangle\left\langle\Psi_{m}^{N-1} \mid \Psi_{i}^{N-1}\right\rangle\right|^{2} .
$$

The change from $\Psi_{f}^{N-1}$ into $\Psi_{m}^{N-1}$, where the states $m$ is the exited state of the final system left 
remaining from the photo-excitation, has been made. Because of the sudden approximation the initial and final states of the system are equal, i.e., $\Psi_{m}^{N-1}=\Psi_{i}^{N-1}$, and therefore makes $w_{f, i} \propto\left|\left\langle\phi_{f}^{k}\left|H_{\text {int }}\right| \phi_{i}^{k}\right\rangle\right|^{2}$

\section{Self Energy and Spectral Function}

Inside a solid the electrons do interact, sometimes strongly with each other. There is a need to write the total photoemission intensity as a summation over all the interacting states in the system for all outgoing kinetic energies $\left(E_{k i n}\right)$ and momenta $(\mathbf{k})$, i.e.,

$$
I\left(\mathbf{k}, E_{k i n}\right)=\sum_{f, i} w_{f, i} \propto \sum_{f, i}\left|\left\langle\phi_{f}^{k}\left|H_{\mathrm{int}}\right| \phi_{i}^{k}\right\rangle\right|^{2} \sum_{m}\left|\left\langle\Psi_{m}^{N-1} \mid \Psi_{i}^{N-1}\right\rangle\right|^{2} \delta\left(E_{k i n}+E_{m}^{N-1}-E_{i}^{N}-h \nu\right)
$$

If $\sum_{m}\left|\left\langle\Psi_{m}^{N-1} \mid \Psi_{i}^{N-1}\right\rangle\right|^{2}$ becomes 1 at a single $m$ and zero everywhere else, as in the case for non interacting particles, and at the same time $\sum_{f, i}\left|\left\langle\phi_{f}^{k}\left|H_{\text {int }}\right| \phi_{i}^{k}\right\rangle\right|^{2}$ does not equal zero, the spectral function will consist of a series of delta functions at a given momentum and energy, Figure 2.3 (b). If, on the other hand, the system is strongly correlated, many of the $\sum_{m}\left|\left\langle\Psi_{m}^{N-1} \mid \Psi_{i}^{N-1}\right\rangle\right|^{2}$ will not equal zero, causing an overlap between many different states. The ARPES intensity will not consist of only delta functions, but in fact will be a convolution between delta functions and interacting states, Figure 2.3 (c). A similar example of the situation is the photoemission of molecular hydrogen, see Figure 2.3 (c) - bottom right [38]. In this case the spectrum is not a single peak, but many peaks separated by few tenth of meV (solid peaks). These peaks correspond to the different modes of vibration in molecular hydrogen. In the case of solid hydrogen, these vibrations would cause a broad continuum with a sharp peak from the transition from the ground state of the $\mathrm{H}_{2}$ to the one of the $\mathrm{H}_{2}+$ molecule (dashed spectrum, Figure 2.3 (c)) [39].

The theoretical formalism that usually describes the single particle spectral function comes from the Green's function formalism [40-43]. The interaction between an electron and a photon in an interacting electro-magnetic field of bands can be written as the total spectral function,

$$
A(k, \omega)=-\frac{1}{\pi} \operatorname{Im} G(k, \omega) .
$$




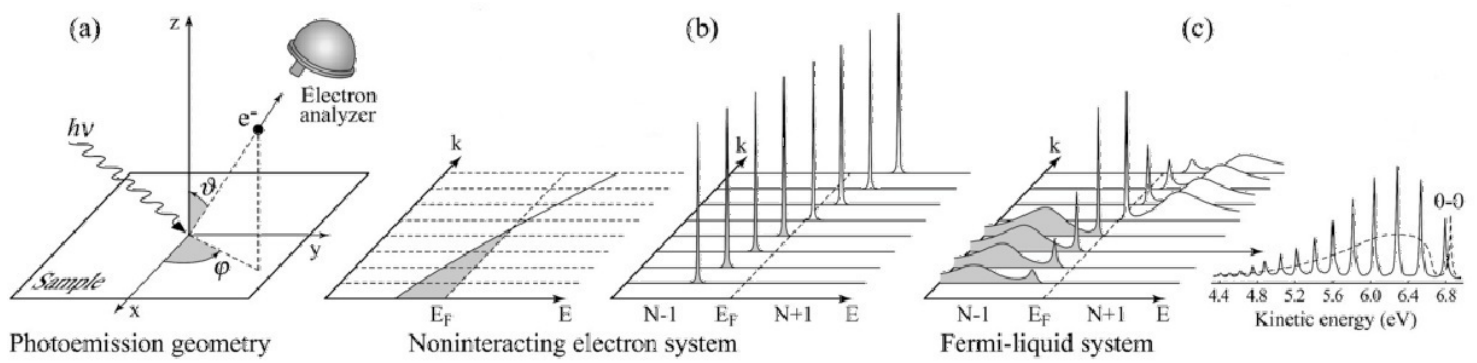

Figure 2.3 Angle-resolved photoemission spectroscopy: (a) the geometry of an ARPES experiment in which the emission direction of the photo-electron is specified by the polar $(\theta)$ and azimuthal $(\varphi)$ angles; (b) momentum-resolved one-electron spectra for a non-interacting electron system with a single energy band dispersing across $E_{f}$ (The spectral function for the non-interacting case is simply a series of delta functions); and (c) the same spectra for an interacting Fermi-liquid system. For both non-interacting and interacting systems, the corresponding ground state $(\mathrm{T}=0 \mathrm{~K})$ momentum distribution function $\mathrm{n}(\mathrm{k})$ is also shown. Lower right in (c), photo-electron spectrum of gaseous hydrogen (solid line) and the ARPES spectrum of solid hydrogen (dashed line) developed from the gaseous one. Figure adapted from Damascelli et al. [32].

In this case the Green function takes the form of

$$
G(k, \omega)=\frac{1}{\omega-\varepsilon_{k}-\sum(k, \omega)}
$$

where $\sum(k, \omega)$ is the self energy of the system and $\varepsilon_{k}$ is the band energy. Note, the formalism of $E_{k i n}-h \nu$ in eq. (2.18) has been exchanged with $\omega$ as the energy of the system. The self energy of the system can be broken into its real and imaginary parts, e.g., $\sum(k, \omega)=$ $\sum^{\prime}(k, \omega)+i \sum^{\prime \prime}(k, \omega)$. In turn this allows the spectral function to be written from Eq.(2.17)

$$
A(k, \omega)=-\frac{1}{\pi} \frac{\sum^{\prime \prime}(k, \omega)}{\left[\omega-\varepsilon_{k}-\sum^{\prime}(k, \omega)\right]^{2}+\left[\sum^{\prime \prime}(k, \omega)\right]^{2}} .
$$

Notice the spectral function takes the form of a Lorentzian. In general, the exact calculation of the spectral function $(A(k, \omega))$ can be difficult because of a lack of knowledge of the self energy $\left(\sum^{\prime \prime}(k, \omega)\right)$. However, self energy can be extracted from experiment, and modeled 
theoretically $[44,45]$. For the purpose of the studies in this dissertation, knowing that the spectral function takes the shape of a Lorentzian for the purpose of band fitting is sufficient.

\section{Bibliography}

[26] H. Hertz, Ann. Physik 31, 983 (1887).

[27] A. Einstein, Annalen der Physik 325, 199 (1906).

[28] D. W. Lynch, and C. G. Olson, Photoemission Studies of High-Temperature Superconductors, Cambridge University Press, Cambridge, Chapter 3 (1999).

[29] S. Hufner, Photo-electron Spectroscopy, Springer-Verlag, Berlin Heidelberg, Chapter 6 $(1995)$.

[30] C. N. Berglund and W. E. Spicer, Phys Rev. A 136, 1030 and 1044 (1964).

[31] P. J. Feibelman and D. E. Eastman, Phys. Rev. B 10, 4932 (1974).

[32] A. Damascelli, Z. Hussain, and Z.-X. Shen, Rev. Mod. Phys. 75, 473 (2003).

[33] M. R. Norman, M. Randeria, H. Ding. and J. C. Campuzano, Phys. Rev. B 59, 11191 (1999).

[34] A. Kaminski, S. Rosenkranz, H. M. Fretwell, J. Mesot, M. Randeria, J. C. Campuzano, M. R. Norman, Z. Z. Li, H. Raffy, T. Sato, T. Takahashi, and K. Kadowaki, Phys. Rev. B 69, 212509 (2004).

[35] M. P. Seah and W. A. Dench, Surf. Interface Anal. 1, 2 (1979).

[36] Terry L. Alford, Leonard C. Feldman, and James W. Mayer, Fundamentals of Nanoscale Film Analysis, Springer, Philadelphia, US, pg. 109 (2007).

[37] J. D. Koralek, J. F. Douglas, N. C. Plumb, Z. Sun, A. V. Fedorov, M. M. Murnane, H. C. Kapteyn, S. T. Cundiff, Y. Aiura, K. Oka, H. Eisaki, and D. S. Dessau, Phys. Rev. Lett. 96, $017005(2006)$. 
[38] K. Siegbahn, ESCA Applied to Free Molecules, North Holland, Amsterdam, (1969).

[39] A. Damascelli, Physica Scripta, 109, 61 (2004).

[40] L. Hedin, and S. Lundqvist, in Solid State Physics: Advances in Research and Applications (edited by H. Ehrenreich, F. Seitz, and D. Turnbull), 23 Academic, New York (1969).

[41] G. Wendin, Breakdown of the One-Electron Pictures in Photo-Electron Spectroscopy, 45 Springer-Verlag, Berlin (1981).

[42] C.-O. Almbladh, and L. Hedin, in Handbook of Synchrotron Radiation I b (edited by D. E. Eastman, Y. Farge, E. -E. Koch) North Holland Publishing Co., Amsterdam pg. 607 (1983).

[43] G. Rickayzen, Green's Functions and Condensed Matter in Techniques of Physics 7, Academic Press, London, (1991).

[44] A. Kaminski, and H. M. Fretwell, New J. Phys. 7, 98 (2005).

[45] M. R. Norman, H. Ding, H. Fretwell, M. Randeria, and J. C. Campuzano, Phys. Rev. B 60, 7585 (1999). 


\section{CHAPTER 3. ANGLE-RESOLVED PHOTOEMISSION SPECTROSCOPY (ARPES): EXPERIMENT}

\section{Introduction}

Before the 1960's almost all photoemission data were exclusively angle-integrated. In 1964 Gobeli et al. showed momentum conservation during the photoemission process and proof that mapping out the complete band structure could be possible [46]. The first angle-resolved photoemission detectors were single point energy detectors that could only find the energy dependence of outgoing electrons. The opening to the detectors allowed only a small angle of electrons to enter; moving the samples through different angles would probe different momenta. To map the full band structure one would have to move through all angles $\theta$ and $\varphi$ defined in Figure 2.3 a). The early ARPES studies on cuprates were mapped in this way [47]. Today ARPES data sets are collected with a 2-D detector (energy and momentum channels), reducing the degrees of freedom, so one only has to change one angle of the sample to map the band structure. New time of flight detectors are being developed to detect all 3 dimensions at once without changing any angles [48]. This chapter reviews experimental setups of a modern ARPES system encompassing both lab and synchrotron-based systems. In addition ARPES data collection and analysis will also be reviewed.

\footnotetext{
ARPES Analyzers

ARPES analyzers collect outgoing electrons within a finite acceptance angle and energy resolution and bin the electrons according to their momentum and kinetic energy. ARPES analyzers consist of three parts: electron lens, which sorts the electrons according to their
} 


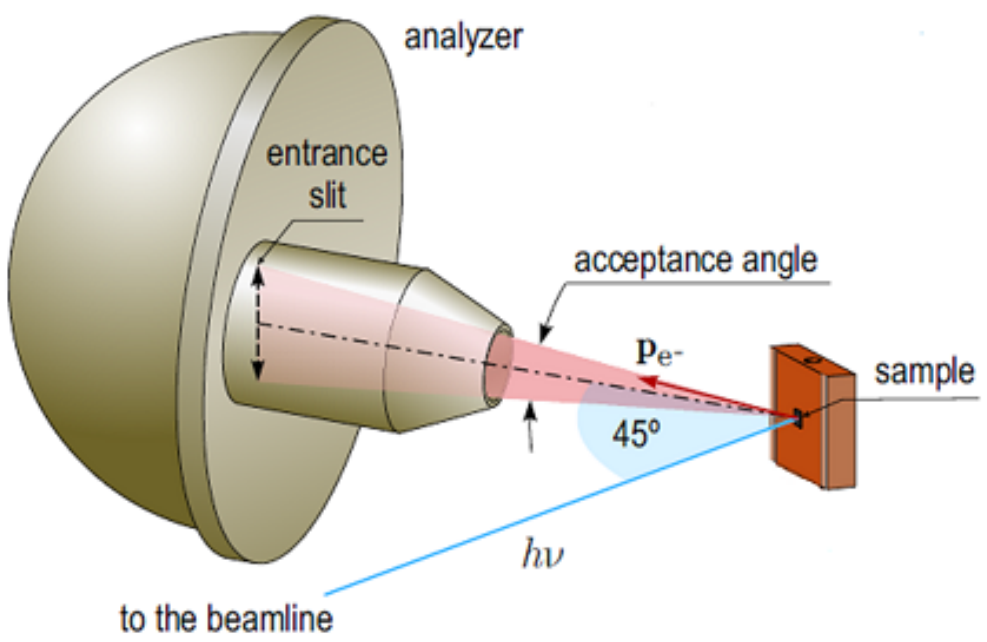

Figure 3.1 Schematic setup of a standard ARPES experiment. The protruding section is the electron lens, attached to the hemispherical energy analyzer. The acceptance angle, beam position, and distance from sample to lens vary with setup. The sample size is exaggerated. Diagram taken from D. S. Inosov [50].

ejection angle; hemispherical energy analyzer, which sorts the electron by their kinetic energy; and 2-D MCP detector, which records the two quantities for each photo-electron. A schematic drawing of a standard photo-emission detector found in most ARPES laboratories is shown in Figure 3.1. A energy resolution of $1 \mathrm{meV}$ and angular resolution of $0.1^{\circ}$ [49] can be achieved using a helium discharge lamp and this performance can be greatly improved when using laser based UV sources — with smaller bandwidths and lower energy. The sample is positioned in front of the lens with a beam of monochromatic light incident on the sample from the side. The acceptance angle depends on the diameter of the lens aperture, the distance from the sample to the entrance slit, and the lens voltages; the further the sample is from the opening, the smaller the acceptance angle must be.

\section{Electron lens}

Once the electrons are ejected from the sample, they travel in a straight line through the vacuum until they reach the entrance slit to the lens. To ensure that the trajectories 


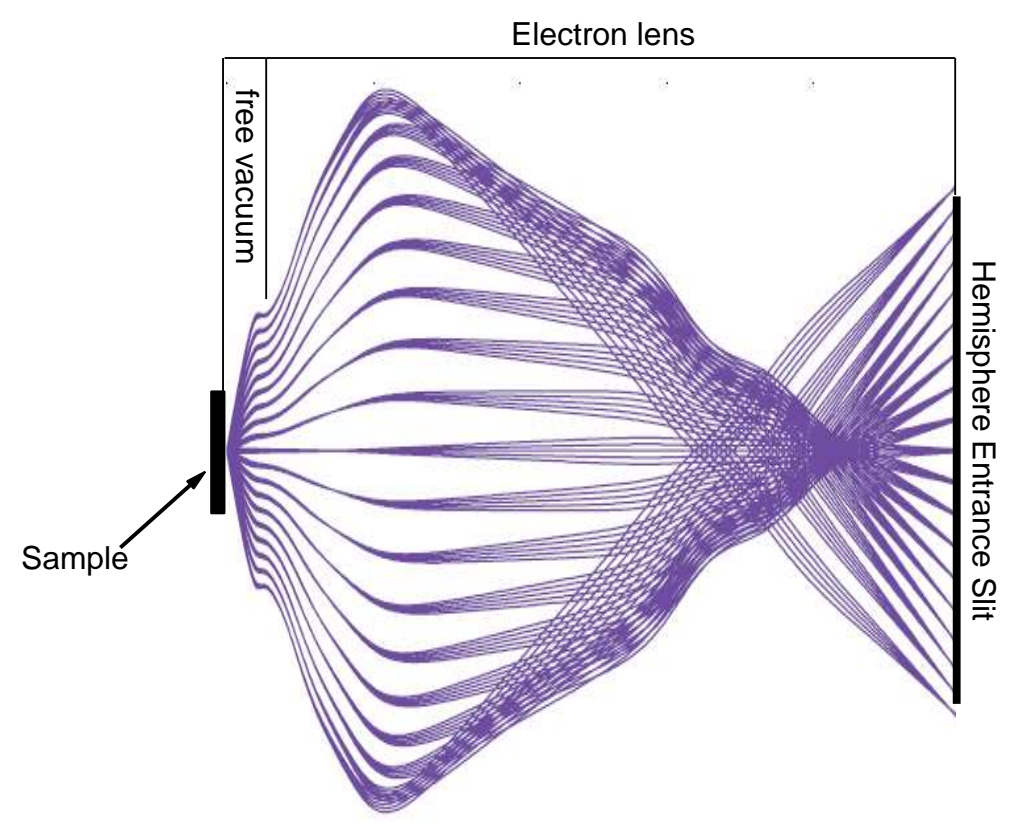

Figure 3.2 A schematic view of the paths of electrons as they move through the electron lens in a modern ARPES analyzer, each line represents the path of a photoelectron at a particular angle from the sample. Figure adapted from VG Scienta [51].

are straight lines, ARPES chambers and analyzers are screened from the Earths and stray magnetic fields using $\mathrm{Mu}$-metal shielding. After the electrons enter the lens, they are focused onto the entrance slit of the hemispherical capacitor. Inside the electrostatic lens metal plates are held a constant potential creating a static lens. The path of the electrons in the lens are shown schematically in Figure 3.2.

\section{Hemispherical analyzer}

After exiting the lens, the electrons enter the hemispherical analyzer. The hemispherical analyzer consists of two metal hemispheres; the two half spheres are arranged in such a way that their centers of curvature are located at the same point. Differential voltages are applied to each hemisphere producing an electric field between the two. As the electrons enter the sphere the faster moving (higher energy) electrons will end up closer to the outer sphere while slower 


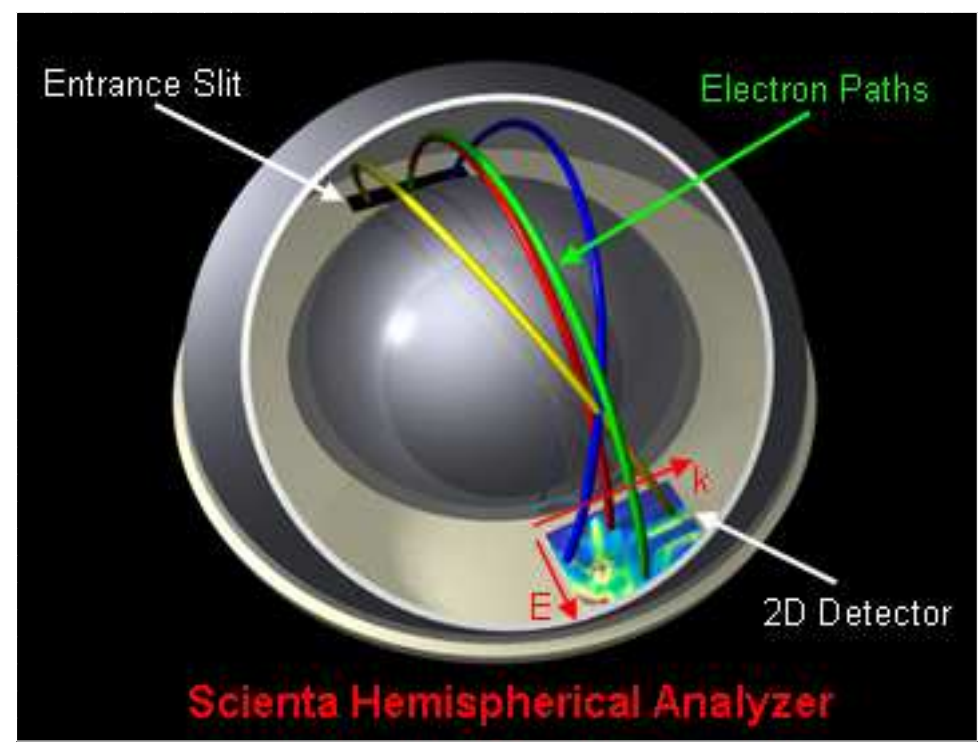

Figure 3.3 A schematic view of the path of the electrons as they move through the entrance slit of the electron lens to the 2-D MCP detector. When the electrons reach the MCP they are multiplied. The multiplied electron packets then excite a small area on a phosphorus screen releasing photons. The light from the phosphorus screen is then recoded by a charge coupled device (ccd) camera. The false color image represents the data collected by the camera. Figure adapted from beamline V-4 of SSRL website at Z.-X. Shen's laboratory [52].

moving (lower energy) electrons will end up closer to the inner sphere. If the energy of the electrons is too high or too low they will hit the walls of the hemispheres before they reach the other side. The energy width of the electrons which make it all the way around the analyzer is called the pass energy. By the time each electron reaches the end of the hemispherical analyzer its' position will place it within a single energy and angle bin. Figure 3.3 shows the electron's path as it moves inside the hemisphere.

\section{2-D MCP detector}

After the electrons travel through the lens and the hemispherical capacitor they are counted by a 2-D detector. The detector is made up of an electron micro channel plates (MCP) coupled to a phosphorus screen positioned in front of a charge-couple-device (CCD) camera (1000 
energy channels and 600 angular channels) [49]. The electron multiplier plate turns a single incident electron into million of electrons through secondary emission. This packet of electrons then hits the screen creating a flash of light. Each individual electron that enters the MCP is multiplied as is passes through the plate. On the far side of the MCP each individual electron is now a packet of electrons in a given energy/momentum bin. This packet hits the phosphorus plate creating a flash of light. The flashes of light are then detected by the camera. Figure 3.3 shows a false color statistically enhanced image the camera takes at the end of the analyzer.

\section{Ultra High Vacuum}

Ultra high vacuum (UHV) is a term that refers to any pressure below $1 \times 10^{-9}$ Torr. To achieve such low pressures, a vacuum chamber must be completely sealed from the outside. This is generally completed by using all stainless steel parts sealed together by soft copper gaskets cut with knife edges into both sides of the gasket and pumped down with multiple pumps. Figure 3.4 is a schematic guide to the location of each component in a UHV system. This section is adapted from the author's knowledge about UHV experiments, knowledge that can also be found in vacuum system manuals from Varian [53] and books such as Scientific Foundations ofVacuum Technique by Dushman [54] and Vacuum Techniques by Rozanov and Hablanian [55].

A roughing pump is the first stage in creating a vacuum. Roughing pumps are able to remove the majority of gases from the chamber down to $1 \times 10^{-3}$ Torr. There are multiple types of roughing pumps, such as rotary pumps, dry scroll pumps, and vein pumps. Roughing pumps are usually used as the first stage for other mechanical pumps which cannot exhaust into atmospheric pressure at full operational speeds.

Turbo molecular pumps contain multiple layers of fixed and rotating fan-like blades. The pump's blades spin at high speeds (up to 80,000 rpm), providing momentum to particles, and removing them from the chamber. Turbo pumps can operate at full speed at pressures from $1 \times 10^{-2}$ Torr to $1 \times 10^{-11}$ Torr. Depending on the application they are used only to reach UHV

during a bake-out, however they can be used to maintain UHV as well. Since turbo pumps are 


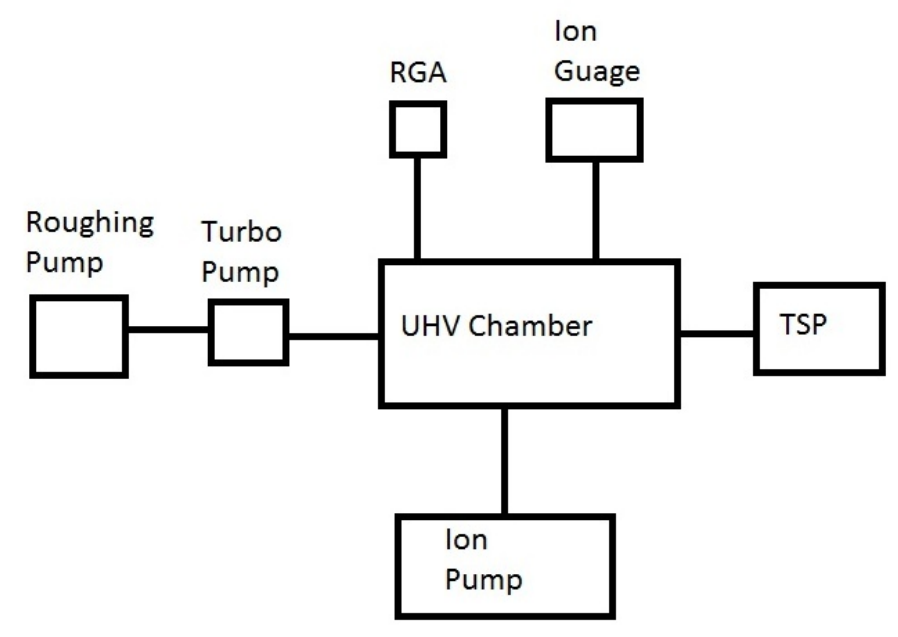

Figure 3.4 Schematic diagram of a UHV setup, each box represents a different component of a complete UHV system and there location about the main chamber, each line represents a connection from one component to another.

not able to operate at full speed exhausting directly into the atmosphere, they usually backed by a roughing pump.

A titanium sublimation pump (TSP) is used to absorb chemically active gases, such as oxygen and water in a vacuum, allowing the vacuum to reach below $1 \times 10^{-12}$ Torr. The pump is a simple cylinder or sphere with a filament of tungsten coated with titanium in the middle. By heating the filament, titanium sublimates onto the walls of the cylinder. This highly reactive layer of titanium bonds with active gases in the vacuum.

Ion pumps are the workhorse of an UHV system, with the ability to lower the pressure in a chamber below $1 \times 10^{-13}$ Torr. Ion pumps contain multiple sheets of titanium and/or tantalum held at high voltage $(3-7 \mathrm{kV})$ clasped by solid state magnets. The high potential field accelerates free ions in the vacuum, while the magnetic field forces the ions to travel in a helical path, ionizing more atoms. When the ions hit the charged plates they sublimate titanium onto the walls, pumping in the same manner as a TPS.

Most modern chambers are made from either stainless steel or aluminum, with the connecting flanges almost exclusively made out of stainless steel. The flanges contain a knife edge 
that cuts into a oxygen free copper gasket to seal the gaps. The gaps may also be sealed by flexible Viton gaskets, but these gaskets usually cannot be heated above $150^{\circ} \mathrm{C}$.

Ion gauges are the pressure detecting workhorse of high and ultra high vacuum systems. The inside of an ion gauge consists of an emission filament, placed outside a helical grid/coil that contain a collecting wire in its' middle. The filament is heated inside the vacuum to a point where it emits a constant current of electrons into the vacuum. The helical grid is held at a positive potential so the electrons from the filament accelerate toward the grid. As the electrons accelerate toward the grid they collide with the gas particles in the vacuum ionizing them. These ionized particles then hit the collector creating a positive current. Like all detectors/gauges, they do have their limits. Ion gauges in particular have a limit of about $3 \times 10^{-11}$ Torr, where x-rays are created when filament electrons hit the grid; the x-rays in turn hit the collector creating photo-electrons which produce a constant negative current.

Unlike an ion gauge designed to detect the total pressure inside a vacuum chamber, residual gas analyzers (RGA) have the ability to detect the components or partial pressure of each gas in a chamber. This is especially important for detecting leaks and contaminants in a vacuum. RGA operate on the principle that one can isolate molecules into their respective charge to mass ratio through a quadruple mass filter.

To reach UHV (below $1 \times 10^{-9}$ Torr), a bake-out is required. During a bake-out, the chamber and some of the pumps are heated to an elevated temperature, where particles are more easily released from the walls of the chamber so they can be effectively pumped out or absorbed. The higher the temperature, the faster is the desorption and more effective the bake-out. However, the usual bake-out temperature is between $100-250{ }^{\circ} \mathrm{C}$ for at least several days. Some portions of the chamber cannot be heated above $150{ }^{\circ} \mathrm{C}$ or sensitive/flexible parts may be damaged. An example of the Ames Laboratory system in a bake-out is shown in Figure 3.5. Figure 3.5 a) is the system completely wrapped in multiple layers of tinfoil, used as an air barrier. Custom oven heaters are mounted on the bottom of the system; the hot air is trapped by the tinfoil heating system by convection. Protruding elements from the chamber that cannot be contained under the dome are wrapped in heater tape and heated through conduction. Figure $3.5 \mathrm{~b}$ ) is 


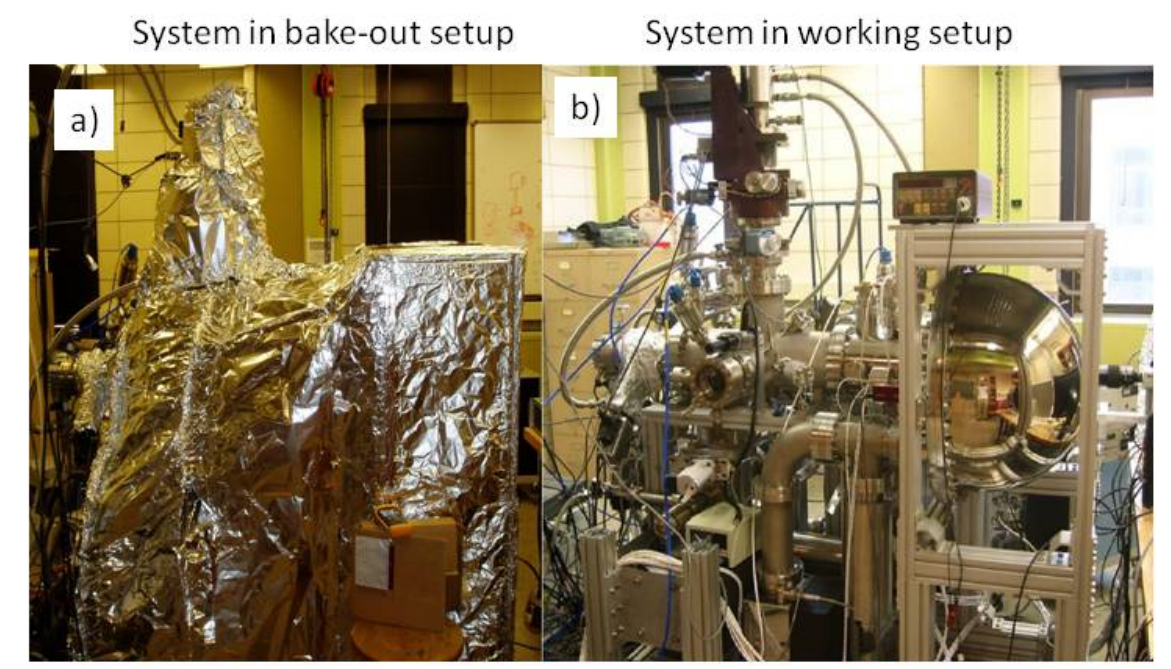

Figure 3.5 ARPES system at Ames Laboratory: a) system during the bake-out procedure wrapped in multiple layers of tinfoil, heated by custom-built oven heater mounted on the bottom of the chamber; b) the same chamber in working condition after a bake-out.

the same system in working condition after a bake-out.

\section{Light Sources}

Modern light sources used in ARPES experiments fall into two main categories: 1) synchrotron light sources and 2) - lab based light sources. Each has its own advantages and disadvantages.

The most common lab-based light source is a UV helium excitation lamp, based on helium plasma generated by electron cyclotron resonance (ECR). The standard off-the-shelf source from VG Scienta is capable of producing $2 \times 10^{16}$ photons $/(\mathrm{sr} \times \mathrm{s})$ with a bandwidth of $1 \mathrm{meV}$ 


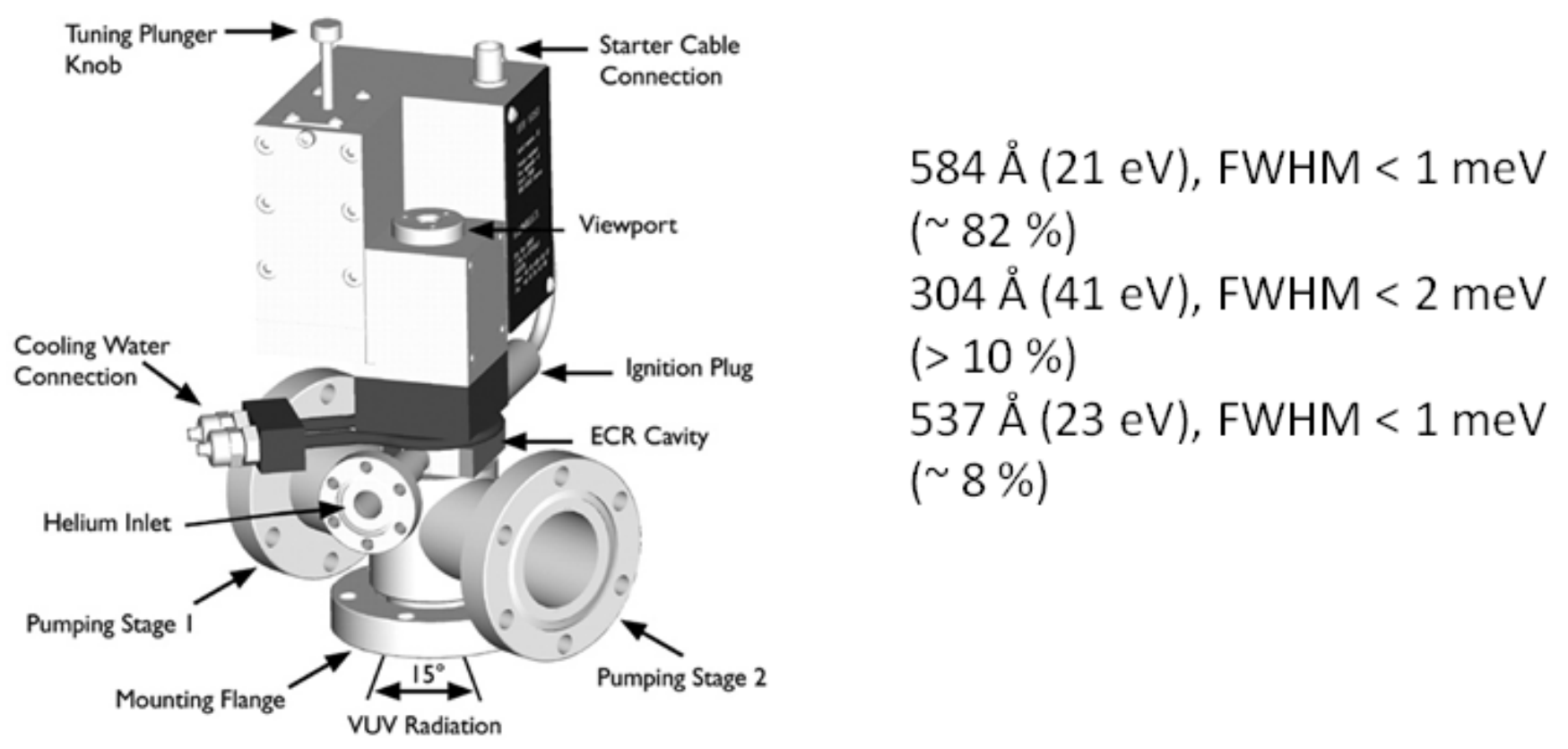

Figure 3.6 Drawing of an off-the-shelf helium discharge lamp produced by VG Scienta model VUV5000. The energy output and bandwidths are listed to the right of the figure [56].

at $21.2 \mathrm{eV}$ (80 percent of light) and $2 \mathrm{meV}$ at $41.8 \mathrm{eV}$ (10 percent of light) [56]. At Ames Laboratory the number of photons that reach the samples is closer to $10^{13}$ photons/s due to the loss from apertures and reflection. A picture of a helium discharge lamp is shown in Figure 3.6. The main advantages of laboratory-based light sources are: a narrow bandwidth, high photon flux, and the ability to collect data 24/7. In addition, laboratory sources are usually not polarized and therefore have at least some access to all the bands. This is because the Matrix element from eq. 2.15 is always greater than zero. The two main disadvantages of laboratory-based systems are 1) lack of ability to tune the photon energy and 2) spot size $(0.5 \mathrm{~mm})$ which is usually an order of magnitude larger than synchrotron sources. Modern laser systems do have the ability to change photon energy and spot size, but this is usually over a small range of energies compared to synchrotron sources. For more information on laser ARPES systems, see J. D. Koralek Dissertation from the University of Colorado, he describes 
the development and implementation of a UV laser in ARPES [57].

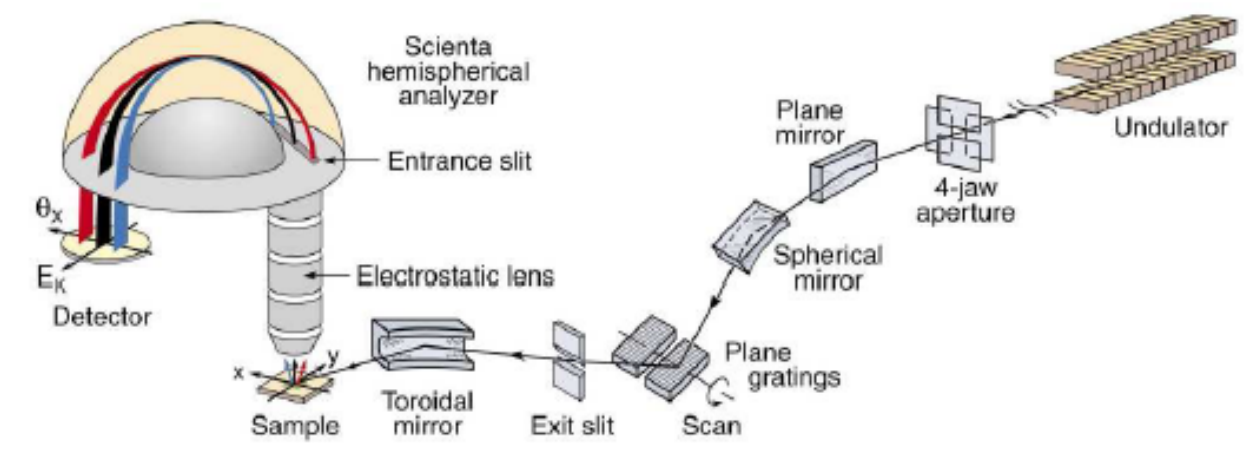

Figure 3.7 A schematic view of a modern synchrotron beam line from right to left, undulator, 4-jaw aperture, mirrors, plane grating (selects energy of the photons), slits and mirror, and finally the sample [58].

Synchrotron sources, unlike lab-based sources, cost upwards of 100 million dollars to build plus significant annual operating costs [59]. Because of this, they are generally set up as a user facility, where visiting scientists apply for beam time at a particular end station. Synchrotron light sources produce photons by accelerating electrons in a storage ring close to the speed of light. Upon bending of the electron beam trajectory in the magnetic field, the electrons emit EM radiation over some range of energies. The emitted photons can then be monochromatized and focused into a small spot. Figure 3.7 shows a diagram of a model ARPES beam-line. Because synchrotrons create photons over a wide range of energies, one needs to select a narrow range of energy with a grating monochromator. The usual energies for ARPES range from 10 to $200 \mathrm{eV}$. There are two experimental disadvantages that most beam lines have; 1 ) The beam is generally highly polarized, when using polarized light some band might not be visible and therefore missed (this can also be an advantage because one can isolate bands with polarized light); 2) The Fermi level tends to shift in time as the beams energy shifts in time, 
especially when new electrons are injected into the ring and the alignment in the accelerator is not identical between the two injections.

At this point, it is worth mentioning that almost all ARPES studies completed to date (including all the studies in this dissertation) are done in a time-integrated manner. That is, a time averaged picture of what is going on in the solid. Currently, there are some groups developing time-resolved techniques with pulsed laser and pulsed linac (linear particle accelerator) free electron laser light sources to gain information on dynamics within the time domain.

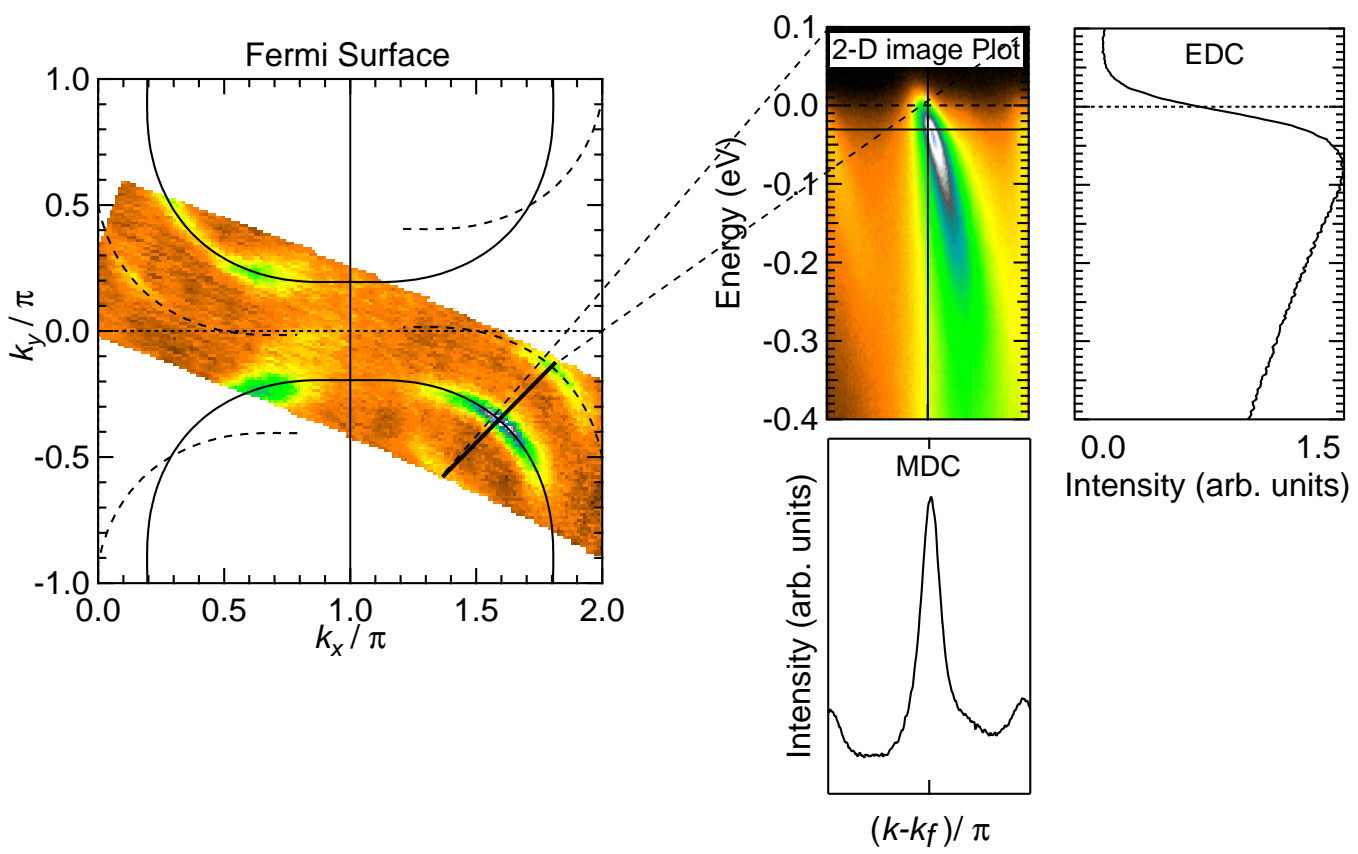

Figure 3.8 A 3-D plot of momentum-energy dispersion of Bi2212. The blue color indicates a higher intensity and black zero intensity moving through the planet earth color scale. This plot depicts different ways to slice though the 3 -D matrix. The Fermi surface (FS) is the contour of high intensity at a constant energy cut at $\mathrm{E}_{F}$, the solid lines represent the fitted FS and the dashed line represent the shadow band. Cutting into/out of the page gives a 2-D image plot (e.g., the actual data recorded by the detector). Vertically cutting through the 2-D image plot creates a single energy distribution curve (EDC). Horizontally cutting through the 2-D image plot creates a momentum distribution curve (MDC). 


\begin{abstract}
ARPES Data
ARPES has the ability to map the full 4-D energy-momentum space for a given material. Figure 3.8 depicts the different ways to cut through and view the 3 -D matrix - a subset of the 4 -D matrix. The Fermi surface is a cut taken at zero binding energy, or if the cut is at a different energy the picture is referred to as a constant energy cut. A 2-D image plot is the actual data obtained from the detector (energy vs. momentum). Taking a vertical cut through the 2-D image plot creates an energy distribution curve (EDC), where the height represented by color intensity in the 2-D image plot. Cutting horizontally through the 2-D image plot creates a momentum distribution curve (MDC), where the height represented by color intensity in the 2-D image plot. In this image plot, the brighter color represents higher intensity and the dark areas represent lower intensities.

To create a full 4-D matrix one would need to map out the full 3-D matrix for a range of incident photon energies, thus building up the 4-D matrix. This is because for a given photon energy, the collected data correspond to a sphere cutting through momentum space. In general, it is not practical to build the complete 4-D matrix; there are two main reasons for this. First, it would take too long to obtain the proper statistics and full 4-D structure. Second, in general, nothing is gained in terms of meaningful physics from taking the full data set. However, taking the full 3-D matrix at a couple of photon energies will give sufficient data to extract meaningful physics. This is exemplified in Figure 3.9 where the Fermi surface of $\mathrm{EuRh}_{2} \mathrm{As}_{2}$ at two different photon energies $105 \mathrm{eV}$ [Figure 3.9 (a)] and $131 \mathrm{eV}$ [Figure 3.9 (b)] are shown. The band dispersion is quite different between the two cuts. The general practice to find the appropriate cutting energies is to scan through the $\mathrm{k}_{z}$ axis by take a cut through the $\Gamma$-X direction, i.e, $\mathrm{k}_{y}=0$ for a wide range of energies to find the symmetry points (FS locations where the photoemission spectrum looks the most different). For the case of $\mathrm{EuRh}_{2} \mathrm{As}_{2}$ in Figure 3.9, $105 \mathrm{eV}$ and $131 \mathrm{eV}$ were close to the symmetry points in the z-axis. After the symmetry points were found, then the 3-D matrix was created [Figure 3.9 (a) and (b)]. In Chapter $2, k_{\|}\left(k_{x}\right.$ and $\left.k_{y}\right)$ were conserved and $k_{\perp}\left(\mathrm{k}_{z}\right)$ was not because of the presence of the inner potential; for this reason alone, the symmetry points cannot be calculated and the
\end{abstract}


inner potential and symmetry point must be extracted from the experimental data.
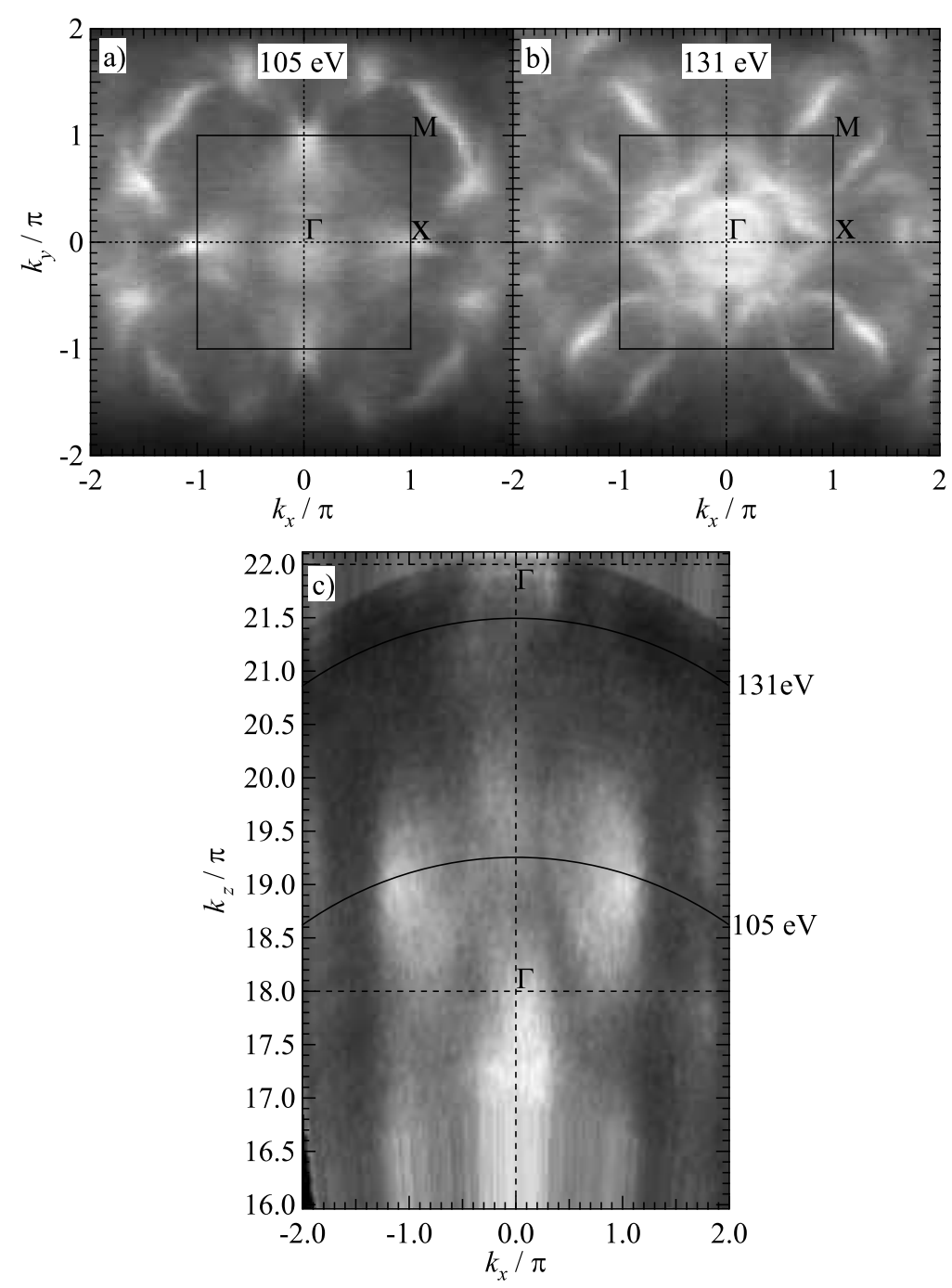

Figure 3.9 Fermi surfaces of $\mathrm{EuRh}_{2} \mathrm{As}_{2}$ integrated within $40 \mathrm{meV}$ of $\mathrm{E}_{F}$ : a) taken with an incident photon energy of $105 \mathrm{eV}$; b) taken with an incident photon energy of $131 \mathrm{eV}$; and c) photon energy $/ k_{z}$ dependence taken at $k_{y}=0$.

\section{Analyzing Data}

Every ARPES data set needs analyzing before the data can represent the band structure. This section will review the minimal analysis needed for all ARPES data sets: Fermi level correction, normalization, and alignment of symmetry axis. 


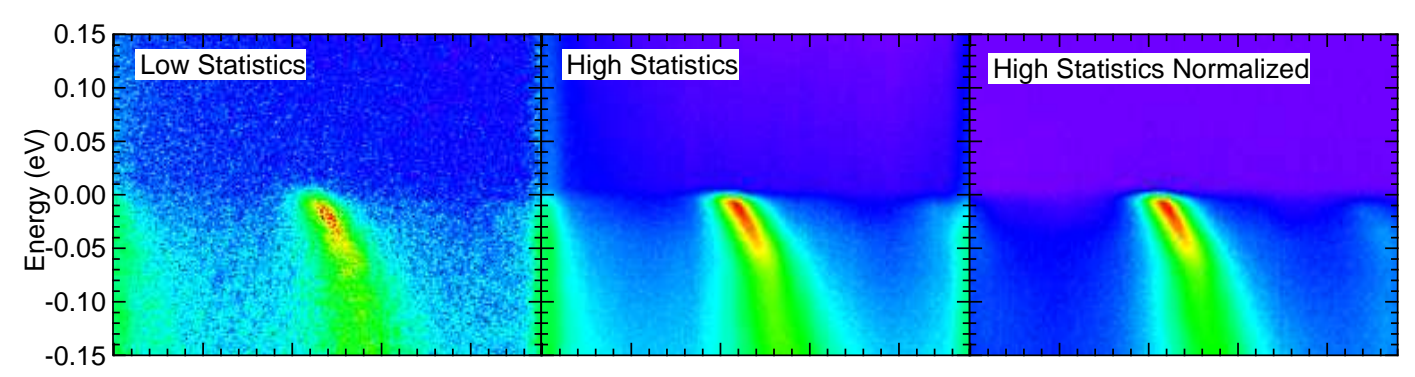

Figure 3.10 Low statistics, high statistics, and high statistics normalized ARPES data, the bottom axis is in units of $\mathrm{k}$ or momentum/angle

\section{Fermi level correction}

When viewing the band structure of a material, one of the most important parameters is the binding energy. This is a problem in ARPES measurements since the detector does not actually measure the binding energy; rather, it measures the electron energy as it relates to the grounding potential of the analyzer. To get the actual binding energy, each channel must be aligned to create a flat cutoff or constant Fermi level. The general practice to accomplish this is to take the angular dependence of the Fermi function (as measured by polycrystalline gold, copper, or aluminum) and determine an offset for each channel. Figure 3.11 plots the position of the Fermi level fitted to the spectrum of polycrystalline gold, as a function of acceptance angle and detector channel taken from a $21.2 \mathrm{eV}$ He II photon source. Depending on the synchrotron beam line the correction may be as large at 20-30 meV. At Ames laboratory the measurements are $16.907 \pm 0.004 \mathrm{eV}$, for most studies using $16.907 \mathrm{eV}$ is fine. However, for studies dividing by the Fermi function (Chapter 7), the exact location for each channel needed.

\section{Normalization data}

ARPES data measured by a 2-D MCP detector inherently has built-in experimental error. These include statistical and detector errors. In general, statistical errors are neglected because of the ability to take longer scans that reduce the error/nose. The next type of error comes from dead pixels, which can be removed manually if needed, or removed in sweep mode by 


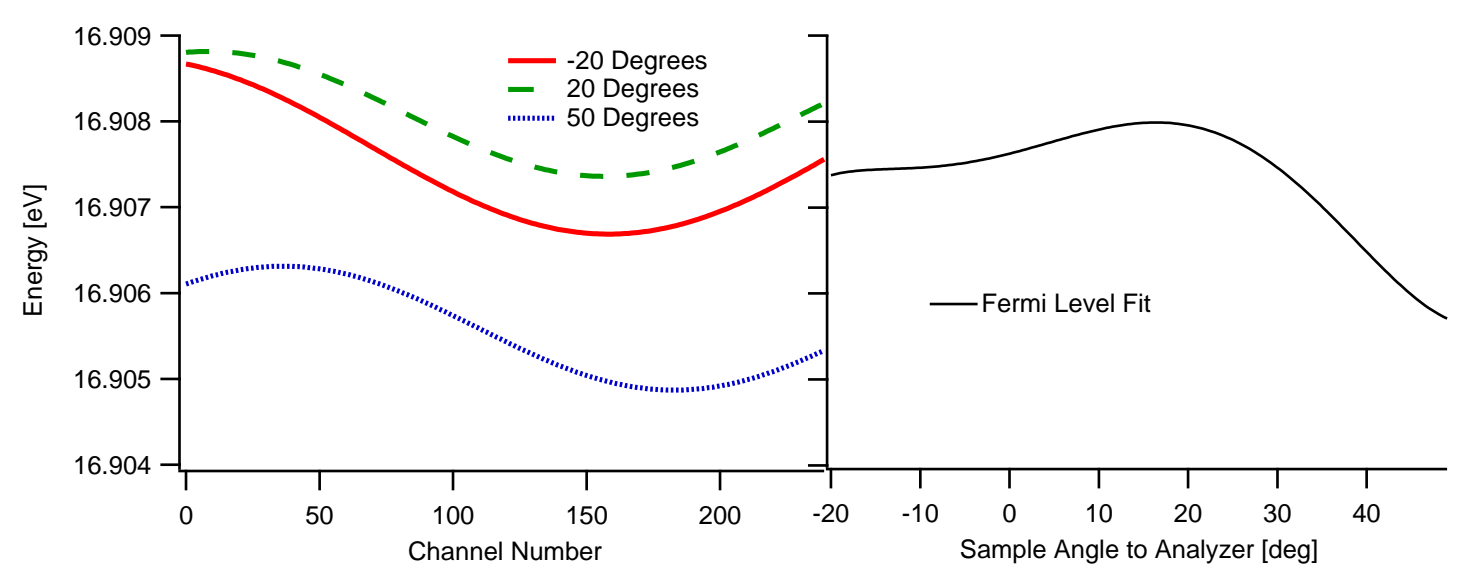

Figure 3.11 Fermi function analysis obtained from polycrystalline gold: left - MCP channel location taken at three different angles (angle between the analyzer central axis and perpendicular to crystal surface) (-20 red, 20 green, and 50 blue); and right Average $\mathrm{E}_{F}$ integrating each channel as a function of angle( the zero angle is defined when the sample plane is perpendicular to the analyzers lens central axis).

normalizing the data of each energy channel. Finally, there are counts above the Fermi level that contribute to the background; these counts will only be present if the beamline and/or lamp has more than one photon energy present. An example of the improved statistics and normalization is shown in Figure 3.10. Ideally, all other data analysis will happen after the normalization of the high statistics data.

In addition to standard normalization, there is an artifact that can come from the phosphorus screen that sometime needs to be removed. In the standard Scienta data acquisition mode, the analyzer scans each pixel through the entire energy range from low energy to high energy. This is done to remove any dead or faint pixels in the MCP. For example, if the desired energy range is from $10 \mathrm{eV}$ to $11 \mathrm{eV}$, and the total energy range of the detector is $0.5 \mathrm{eV}$, then the analyzer scans from $9.5 \mathrm{eV}$ to $11.5 \mathrm{eV}$ dropping the $0.5 \mathrm{eV}$ at the ends after the scan. 

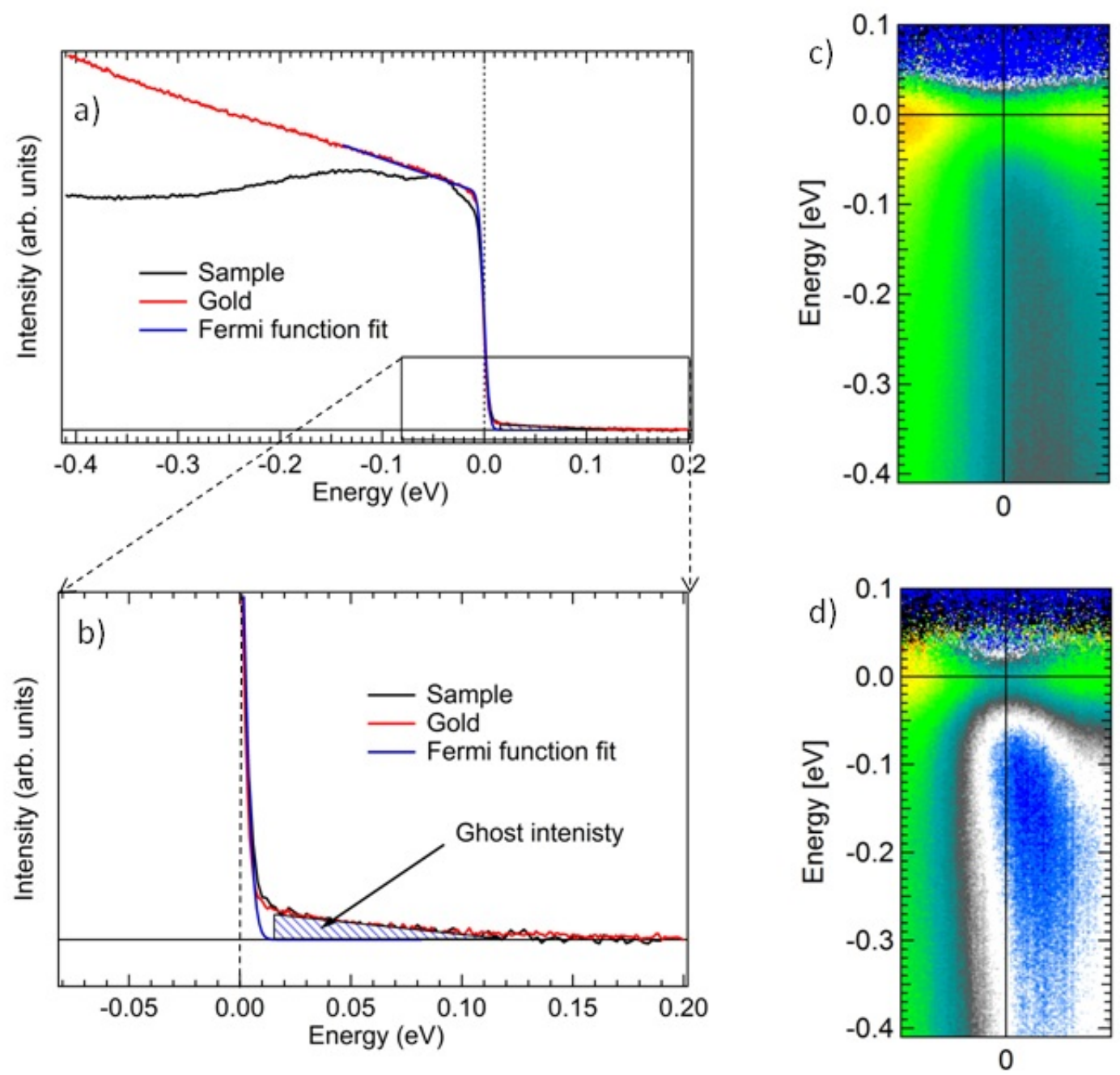

Figure 3.12 (a) EDC cut for a sample (black line Bi2212), gold (red line), and Fermi Function fit (Blue line) at 12K; (b) the same as (a) only zoomed in close to $\mathrm{E}_{f}$, the blue slashed triangle is the ghost region created by scanning mode; (c) 2-D image plot of Fermi divided Bi2212 close to $(\pi, 0)$ with the ghost spectrum left in at $140 \mathrm{~K}$; and (d) the same as (c) only with the ghost intensity removed. For the color scale the blue is the highest intensity, and black zero moving through the planet earth color scale. 

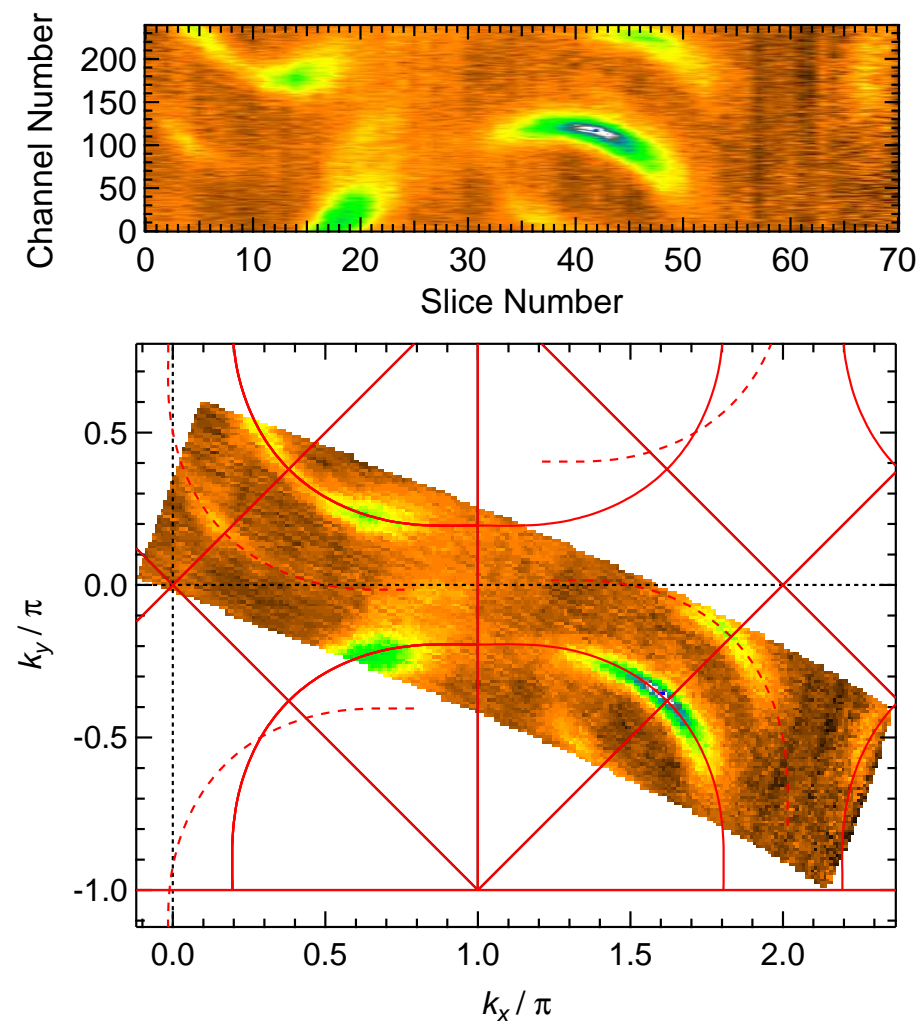

Figure 3.13 Fermi surface of OP90K Bi2212 measured at $12 \mathrm{~K}$ over two Brillouin zones with a $21.2 \mathrm{eV}$ helium excitation lamp. The top figure is the raw data measured by the analyzer. Each slice number represents a single angle bin; the actual angles were from -20 degrees to 50 degrees. The bottom figure is after rotating and scaling the data matrix to orientate the symmetry axises.

One of the artifacts which can appear happens at the Fermi level. In this region, ghost counts can contaminate the spectrum above the Fermi level, probably arising from residual light left on the phosphorus screen. The residual light comes from the fact that the refresh rate of the camera is faster than the refresh rate of the phosphorus screen. This is seen in Figure 3.12 where the EDC from gold and Bi2212 at $12 \mathrm{~K}$ is shown with the Fermi function fit to the gold spectrum. Zooming into the region close to $\mathrm{E}_{f}$ the experimental artifact becomes clear. The ghost intensity region survives up to $0.1 \mathrm{eV}$ and appears to be linear with energy. If this region is not removed before dividing by the Fermi function (Fermi division) [Figure 3.12 
(c)], the data becomes unphysical with the spectra above the Fermi level going to infinity. If the ghost region is removed before Fermi division, the real spectrum is revealed [Figure 3.12 (d)]. In general, most experiments do not care about the band above the Fermi level, so removing this artifact is not important; however, for studies dividing by the Fermi function (Chapter 7), proper subtraction is vital to getting salid data.

\section{Alignment of symmetry axis}

When a sample is placed inside an ARPES chamber, the exact orientation in momentum space is not always known. Even if it is known, the sample is usually not oriented perfectly. For this reason, after taking any data set the matrix must be rotated inside software to align the sample. This is performed through an Euler rotation of the Fermi surface matrix. This rotation maps the flat/skewed data onto the real momentum space sphere. An example is shown in Figure 3.13. The top panel is the raw data showing the momentum space orientation of the sample in the chamber. The bottom panel is the raw data after rotating to align with the symmetry axis, and scaling the data to its correct momentum space representation.

\section{Bibliography}

[46] G. W. Gobeli, F. G. Allen, and E. O. Kane, Phys. Rev. Lett. 12, 94 (1964).

[47] D. W. Lynch and C. G. Olson, Photoemission Studies of High-Temperature Superconductors, Cambridge University Press, Cambridge, Chapter 6-9 (1999).

[48] A. S. Tremsin, G. V. Lebedev, O. H. W. Siegmund, J. V. Vallerga, J. B. McPhate and Z. Hussain, Nuclear Instruments and Methods in Physics Research Section A: Accelerators, Spectrometers, Detectors and Associated Equipment 582, 172 (2007).

[49] Electron Spectrometer Scienta R4000; http://vgscienta.com/ (March 28th 2010).

[50] D. S. Inosov, arXiv: 0807.1434v1 (2008).

[51] http://arpes.stanford.edu/facilities_ssrl.html (March 29th 2010). 
[52] G. hrwall, P. Karlsson, P. Andersson, M. Lundwall, R. Moberg, B. Wannberg, S. Svensson, and M Lundqvist, http://www.vgscienta.com/_resources/File/ARTOF\%20principles\%20 poster\%202008\%20web.pdf (March 28th 2010).

[53] Varian Training Department, Basic Vacuum Practices, Third edition (Varian Associates Inc., Lexington, MA 1992).

[54] S Dushman, Scientific Foundations of Vacuum Techniques (John Wiley and Sons, New York, NY 1949).

[55] L. N. Rozanov and M. H. Hablanian, Vacuum Techniques (Taylor \& Francis, New York, NY 2002).

[56] VUV5000 UV Source, http://www.vgscienta.com/productlist.aspx?MID=19\&IID=429 (April 12th 2010).

[57] J. D. Koralek, Laser Based Angle-Resolved Photoemission Spectroscopy and High Tc Superconductivity, Unpublished PhD dissertation, University of Colorado (2006).

[58] Andrea Damascelli, http://www.physics.ubc.ca/ quantmat/ARPES/PRESENTATIONS /Lectures/ARPES_USC.pdf (November 22th 2010).

[59] W. Eberhardt et al., The JLAMP VUV/Soft X-ray User Facility, Proposal by Thomas Jefferson National Accelerator Facility submitted to the U. S. Department of Energy, http://www.jlab.org/FEL/ (2009). 


\section{CHAPTER 4. CUPRATE SUPERCONDUCTORS}

\section{Introduction}

Superconductivity was first discovered in 1911 by Heike Kammerlingh Onnes in elemental mercury; after his successful liquefaction of helium [60]. Onnes observed the resistivity of mercury dropped to zero upon cooling below the transition temperature $T_{c}=4.2 \mathrm{~K}$. A reproduction of his iconic plot is shown in Figure 4.1. For 20 years, essentially nothing else was known about superconductivity except at temperatures close to absolute zero, the resistance drops to zero. Then in $1933 \mathrm{~W}$. Meissner and R. Ochsenfeld demonstrated a new feature of the superconducting state - perfect diamagnetism or the complete expulsion of magnetic fields from inside the sample $[61,62]$. In their experiment, a hollow cylinder of lead was placed inside a magnetic field; upon cooling below the transition temperature an increase of magnetic field inside the cylinder was observed, after removing the magnetic field in the superconducting state the field inside the superconductor did not change. These two characteristics, zero resistance and the expulsion of magnetic flux, are the hallmarks of superconductivity.

Until the mid 1930s, there was little theoretical advancement towards understanding superconductivity. This changed with phenomenological work by the London brothers, where they explained the electrodynamics and Meissner effect in superconductors [63]. Two decades later Ginzburg and Landau created another phenomenological theory to explain the second order phase transition at $T_{c}$ [64]. While Ginzburg-Landau theory is a phenomenological theory, a microscopic theory of superconductivity was needed. This theory was purposed in 1957 in three papers by J. Bardeen, L. N. Cooper, and J. R. Schrieffer (BCS theory) [65-67]. For most of the next three decades superconductivity was seen as solved. Then in 1986 the field of superconductivity changed with the discovery of a $\mathrm{La}_{(2-x)} \mathrm{Ba}_{x} \mathrm{CuO}_{4} T_{c} \approx 36 \mathrm{~K}$ as a possible 


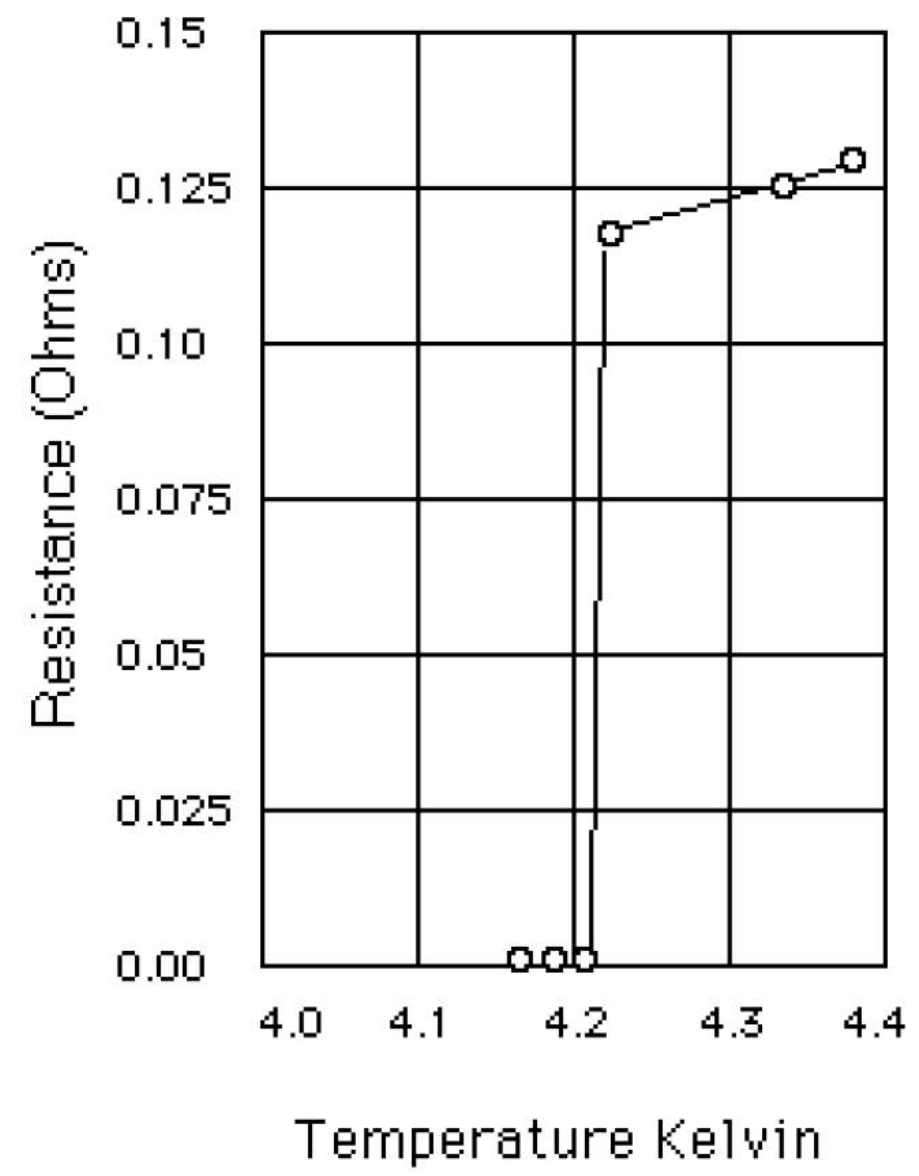

Figure 4.1 Resistance vs. temperature for elemental mercury [75]. 


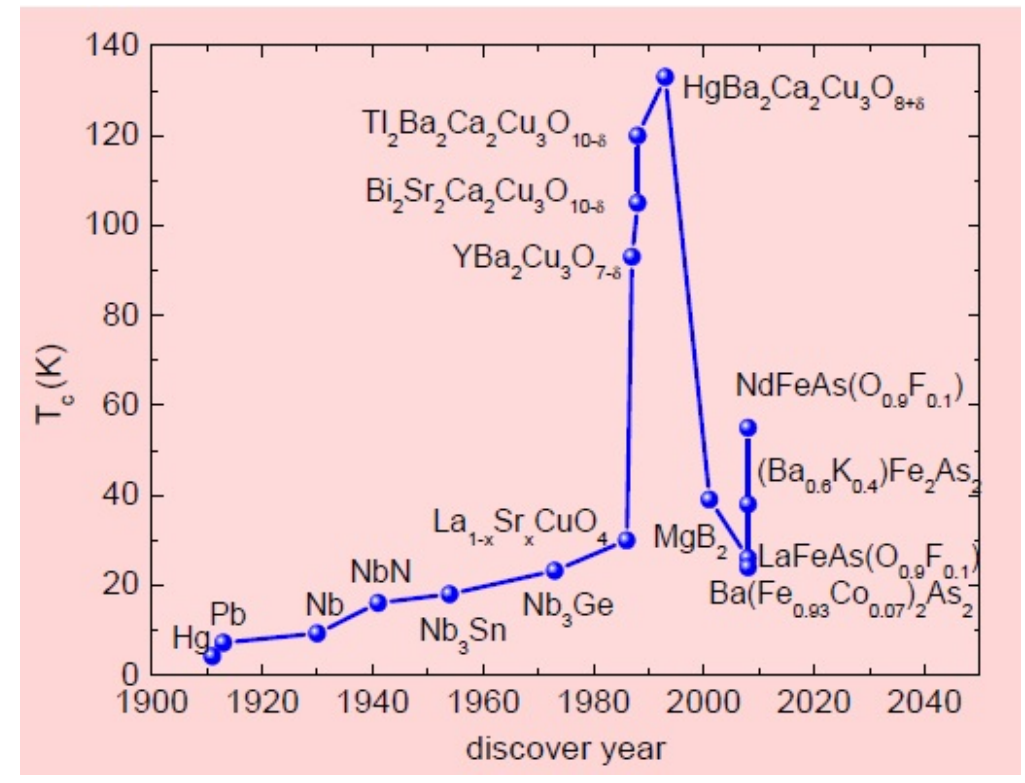

Figure 4.2 Transition temperature vs. discovery year for common superconductors, taken from $\mathrm{Ni} \mathrm{Ni}[75]$.

high temperature superconductor $[68,69]$. Unlike conventional superconductors, which are relatively good conductors, $\mathrm{LaBaCuO}$ is a poor conductor in the normal state. In addition cuprate superconductors ended up having transition temperatures much higher than conventional BCS superconductors, with the onset of $T_{c}$ reaching above $164 \mathrm{~K}$ under pressure [70]. In addition to BCS superconductors and high temperature superconducting cuprates, there are many other families of superconductors with relatively high $T_{c, \max }$. First, $\mathrm{MgB}_{2}, T_{c}=39 \mathrm{~K}$, a two gap superconductors discovered in 2001 [71]. Second, the $1111\left(\mathrm{LaFeAs}\left(\mathrm{O}_{1-x} \mathrm{~F}_{x}\right) T_{c}=26\right.$ $\left.\mathrm{K}[72], T_{c, \max }=55 \mathrm{~K}[73]\right)$ and $122\left(\left(\mathrm{Ba}_{1-x} \mathrm{~K}_{x}\right) \mathrm{Fe}_{2} \mathrm{As}_{2}\right), T_{c, \max }=38 \mathrm{~K}$ [74]) superconducting pnictides were both discovered in 2008. The time evolution of superconducting transition temperatures for various compounds is shown in Figure 4.2.

This chapter will review the triumph of BCS it pertains to classical superconductors, including the physics characteristics which BCS theory explains. Then an overview of cuprate superconductors will be presented, including crystal structure, phase diagram, Fermi surfaces (FS), and gap structures. This chapter will focus primarily on ARPES experiments as it will help in the understanding of the three studies in Chapters 5, 6, and 7. 


\section{BCS}

The first BCS theory paper was written by Cooper [65]. In his paper Cooper set out to explain how at low temperatures a volume-independent gap, $2 \Delta \approx k T_{c}$, between the ground and first excited state in an electron spectrum can form. He considered a pair of electrons that could interact about a "quiescent Fermi sphere." This interaction might be expected from a phonon within a screened Coulomb field. If their net attraction was positive, the two electrons could form a bound state (Cooper pair). The properties of a non-interacting system in which these bound states exist could produce superconductivity. This paper set the groundwork for the theory where a boson pair could exist in a Fermi sea. A simplified picture of such a theory can be conceptualized by thinking about a single electron moving through a lattice of ions. As the electron passes by the ions, the Coulomb force slightly distorts the lattice. This lattice distortion then attracts a new electron, effectively coupling them together. If the attraction is stronger than the Coulomb force between the two electrons, they pair up and form a Cooper pair.

The second paper in support of BCS was written by Bardeen, Cooper, and Schrieffer [66]. They stated the need for a clear mathematical explanation of superconductivity that takes into account the isotope effect; the first paper by Cooper helped set the mathematical groundwork. The isotope effect was the first indicator for phonon mediated superconductivity. The isotope

effect showed a linear scaling between $T_{c}$ and $M^{-\frac{1}{2}}$ where $M$ is the mass of the isotope. Similar to the simplified picture described in the paragraph above; the heavier the ions the smaller the lattice distortion, the smaller the lattice distortion the lower the coupling. Therefore, reducing the transition temperature as the mass goes up [77]. Based on these ideas Bardeen, Cooper, and Schrieffer were able to calculate some of the most important experimental consequences of superconductivity: the Meissner effect, the size of the energy gap and the isotope effect.

The final paper in the series appropriately titled The Theory of Superconductivity [67] solidifies the other two papers $[65,66]$ and presented calculations of the five major experimental features of superconductivity from first principle calculations and also gave quantitative agreement with two others. BCS explained the second-order phase transition at the critical 
temperature, the electronic specific heat varying as $\exp \left(-T_{c} / \mathrm{T}\right)$ near $\mathrm{T}=0$ (evidence of a energy gap), the Meissner effect, infinite conductivity, and the isotope effect. BCS was also able to get good agreement with the actual specific heat and penetration depth experiments with the help of experimentally-determined parameters.

\section{High Temperature Superconducting Cuprates}

Since the discovery of $\mathrm{La}_{2-x} \mathrm{Ba}_{x} \mathrm{Cu}_{4}$ by Bednoz and Muller [68], a relatively large number of superconducting cuprates have been discovered and studied. These high temperature superconducting cuprates (HTSC) have become some of the most studied materials because of there high transition temperatures compared to traditional/BCS superconductors and there very unusual physics originating from their strongly correlated electronic structure. The common feature all high-temperature superconductors share is a layered perovskite tetragonal/orthorhombic crystal structure containing $\mathrm{CuO}_{2}$ planes separated by charge reservoir layers. In the undoped state, the square $\mathrm{CuO}_{2}$ plane has one hole per $\mathrm{Cu}$ atom, which should be a conventional conductor, but is insulating due to strong electron repulsion (Mott insulating state). To dope the samples, ions are added or substituted to the charge reservoir layers, and these ions draw holes or electrons from the $\mathrm{CuO}_{2}$ planes; or doping can be controlled by adding extra oxygen in the $\mathrm{CuO}$ planes as in the Bi family of cuprates [76].

\section{Crystal structure}

All cuprates share approximately the same basic tetragonal/orthorhombic crystal structure. They all have a relatively large c-axis to a/b plane ratio e.g., 3 to 1 in $\mathrm{YBa}_{2} \mathrm{Cu}_{3} \mathrm{O}_{7+\delta}$ (YBCO) and 6 to 1 in $\mathrm{Bi}_{2} \mathrm{Sr}_{2} \mathrm{CaCu}_{2} \mathrm{O}_{8+\delta}(\mathrm{Bi} 2212)$. These quasi-2D materials contain separate planes of either conducting (upon doping) $\mathrm{CuO}_{2}$ or insulating (charged reservoir upon doping) $\mathrm{A}_{(1,2)} \mathrm{O}_{2}$ $\left(\mathrm{A}=\right.$ metal) laying perpendicular to the c-axis, e.g., $\mathrm{Bi}_{2} \mathrm{Sr}_{2} \mathrm{CuO}_{6+\delta}$ has $\mathrm{CuO}_{2}$ layers, $\mathrm{Bi}_{2} \mathrm{O}_{2}$ layers and $\mathrm{Sr}_{2} \mathrm{O}_{2}$ layers. An idealized crystal structure of bismuth based cuprates is shown in Figure 4.3 [79]. Moving from left to right in Figure 4.3, $\mathrm{Bi}_{2} \mathrm{Sr}_{2} \mathrm{CuO}_{6+\delta}(\mathrm{Bi2201})$ has one $\mathrm{Cu}-$ O layer, $\mathrm{Bi}_{2} \mathrm{Sr}_{2} \mathrm{CaCu}_{2} \mathrm{O}_{8+\delta}(\mathrm{Bi} 2212)$ has two $\mathrm{Cu}-\mathrm{O}$ layers, and $\mathrm{Bi}_{2} \mathrm{Sr}_{2} \mathrm{Ca}_{2} \mathrm{Cu}_{2} \mathrm{O}_{10+\delta}(\mathrm{Bi} 2223)$ 

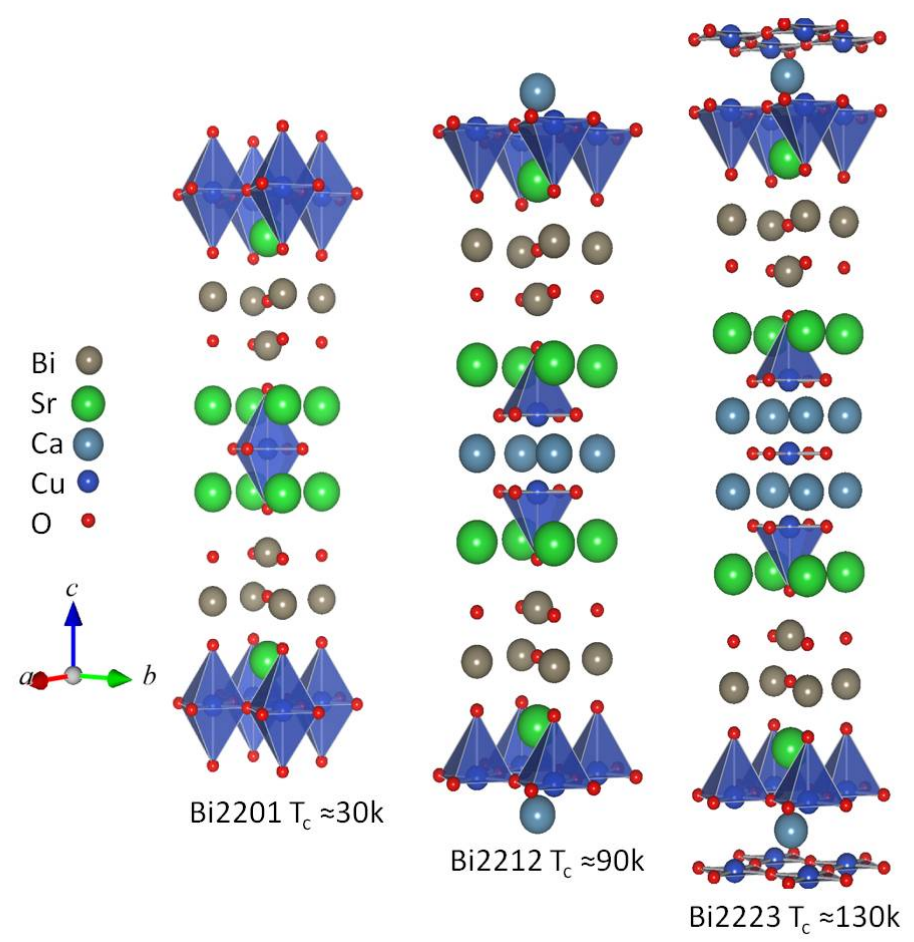

Figure 4.3 Idealized crystal structure of $\mathrm{Bi}_{2} \mathrm{Sr}_{2} \mathrm{CuO}_{6+\delta}, \mathrm{Bi}_{2} \mathrm{Sr}_{2} \mathrm{CaCu}_{2} \mathrm{O}_{8+\delta}$, and $\mathrm{Bi}_{2} \mathrm{Sr}_{2} \mathrm{Ca}_{2} \mathrm{Cu}_{2} \mathrm{O}_{10+\delta}$. The light brown sphere represents bismuth, the green sphere-strontium, the gray sphere-calcium, the blue sphere-copper, and the small red sphere-oxygen. The blue pyramids represent the bonding within the copper oxygen planes. The approximate maximum transition temperature of each optimally-doped compound is stated below each structure. Designed in VIC-II [78].

has three $\mathrm{Cu}-\mathrm{O}$ layers per unit cell. With each additional layer the transition temperature increases up to a maximum of three layers. Other families of cuprates such as thallium bases $\mathrm{Tl}_{2} \mathrm{Ba}_{2} \mathrm{CuO}_{6+\delta}$ (Tl2201), and mercury based $\mathrm{HgBa}_{2} \mathrm{CuO}_{4+\delta}(\mathrm{Hg} 1201)$ have $T_{c, \text { max }}$ closer to $90 \mathrm{~K}$ with only one layer, but, they too, reach a $T_{c, \max }$ at three layers. YBCO is slightly different than other cuprate superconductor because it has both $\mathrm{CuO}_{2}$ planes and $\mathrm{CuO}$ chains; the $T_{c, \max }$ for the family is $100 \mathrm{~K}$ [80]. It was shown by Kondo et al. in 2007 that the $\mathrm{CuO}$ chains do not play a role in superconductivity [81]. 


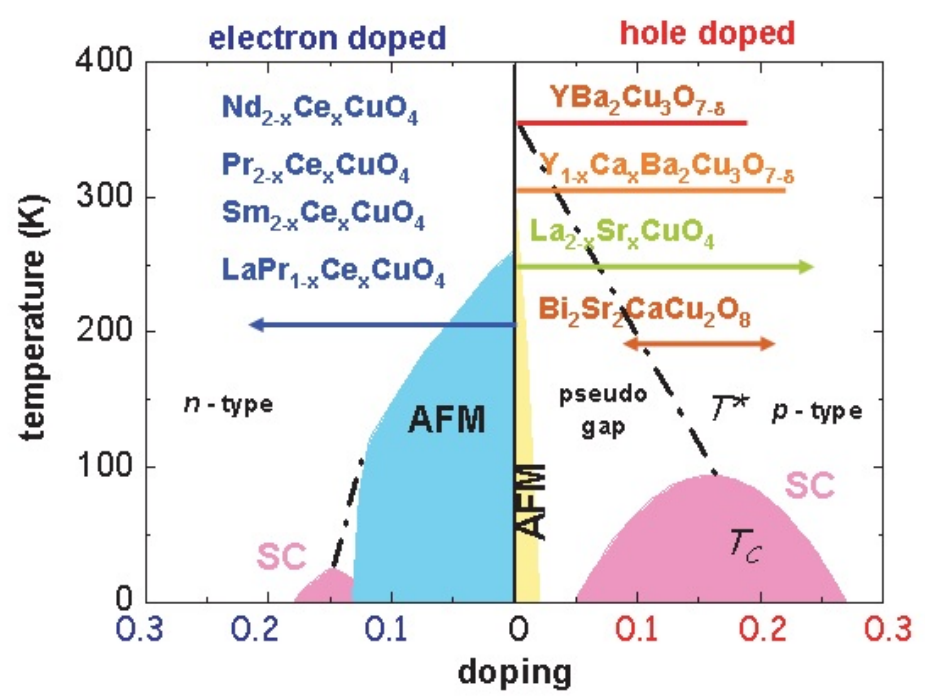

Figure 4.4 Schematic doping phase diagram for both $\mathbf{n}$ (electron-doped) and $\mathbf{p}$ (hole-doped) cuprate superconductors. The doping range for selected materials are shown by the horizontal arrows [82].

\section{Phase diagram}

Superconducting cuprates are non-stoichiometric. In their stoichiometric/near stoichiometric state, the materials are antiferromagnetic insulators. Upon doping, the Néel temperature drops to zero, the pseudogap phase appears, and superconductivity emerges. Upon further doping, the compounds become metallic. An idealized phase diagram of multiple cuprates is shown in Figure 4.4 [82]. Not all materials cover the entire range in doping, for this reason one needs to study different cuprate families. Because of their availability, ease of cleaving, and quality of samples, Bi2201 and Bi2212 are the two most studied materials, especially by ARPES and STM. There are two characteristic temperatures that all hole-doped cuprates share. One is the pseudogap temperature $T^{*}$ and the other is the superconducting transition temperature $T_{c}$. For each compound $T^{*}$ and $T_{c}$ are different, and for each family height in temperature and width in doping for each phase can be different. Both temperatures are shown in Figure 4.4. Depending on the particular sample there may be no pseudogap (i.e., highly over-doped Bi2212-doping around 25\%), or they won't superconduct (i.e. highly underdoped Bi2201-doping around \%5). 

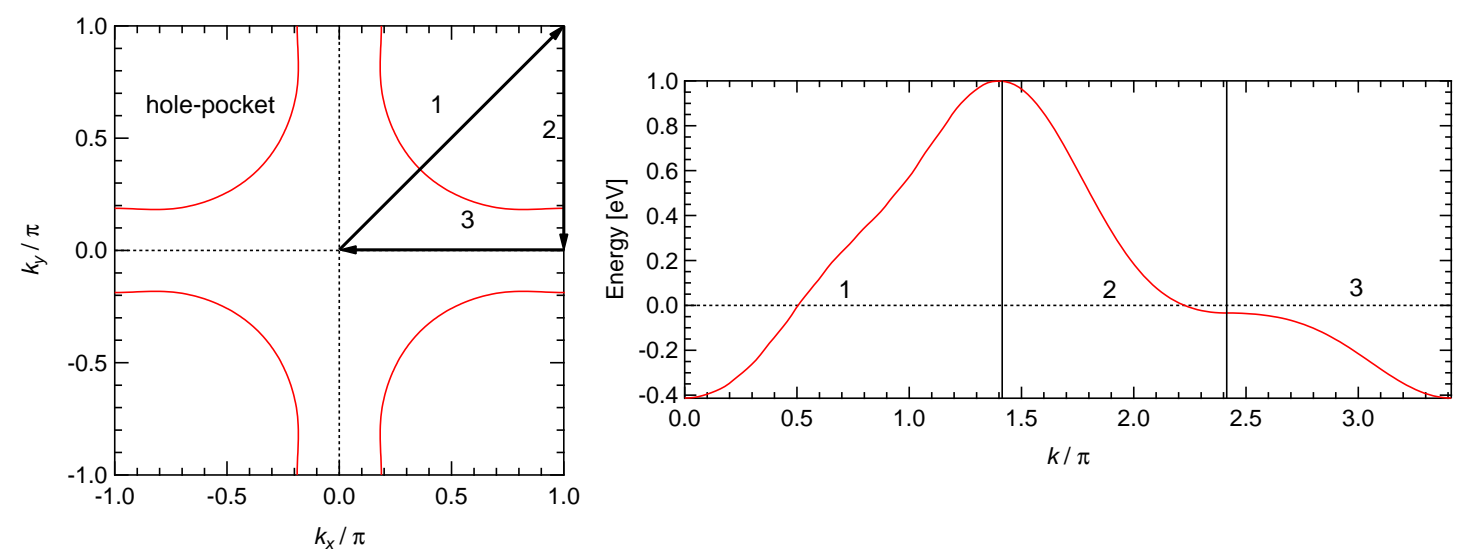

Figure 4.5 Calculated band structure of optimally doped Bi2212: left Fermi surface; and right - cuts along the FS showing the shape of the energy bands.

\section{Cuprate Fermi surface}

All cuprates share a common shaped Fermi surface (FS) arising from the conducting $\mathrm{Cu}-\mathrm{O}$ plane. The idealized FS of the $\mathrm{Cu}-\mathrm{O}$ plane consists of four hole-pockets at the corner of the zone, shown in Figure 4.5. The plot on the right shows the energy-momentum contour plot cutting into the page from $(0,0)$ to $(\pi, \pi) \mathbf{1}$, from $(\pi, \pi)$ to $(0, \pi) \mathbf{2}$, and $(0, \pi)$ to $(0,0) \mathbf{3}$. In most Photoemission papers including this dissertation the units are in $(k \times a) / \pi$, by setting the lattice constant $\mathrm{a}=1$. When doping the size and shape of the FS can change. Underdoping the sample the hole-pockets becomes smaller; overdoping the sample the hole-pockets become bigger. Eventually doping to high levels, the FS at $(0, \pi)$ closes and forms an electron-like band centered at $(0,0)[83]$.

There are other FS characteristics that appear in a number of cuprates. One is the so-called umklapp bands shown in Figure 4.6, dashed blue left side. The umklapp bands come from the modulation of the bismuth layer that resides at the surface of the crystal after cleaving. This modulation acts as a diffraction grating at the surface. As the photoelectron leave the sample some are diffracted at $\pm 0.21 k / \pi$ to the main band in the $(\pi, \pi)$ direction of the $\mathrm{b}$ plane. The general practice in ARPES is to only take data in the zones where the umklapp bands straddles the FS rather than crossing it, e.g., upper left and lower right. This band 


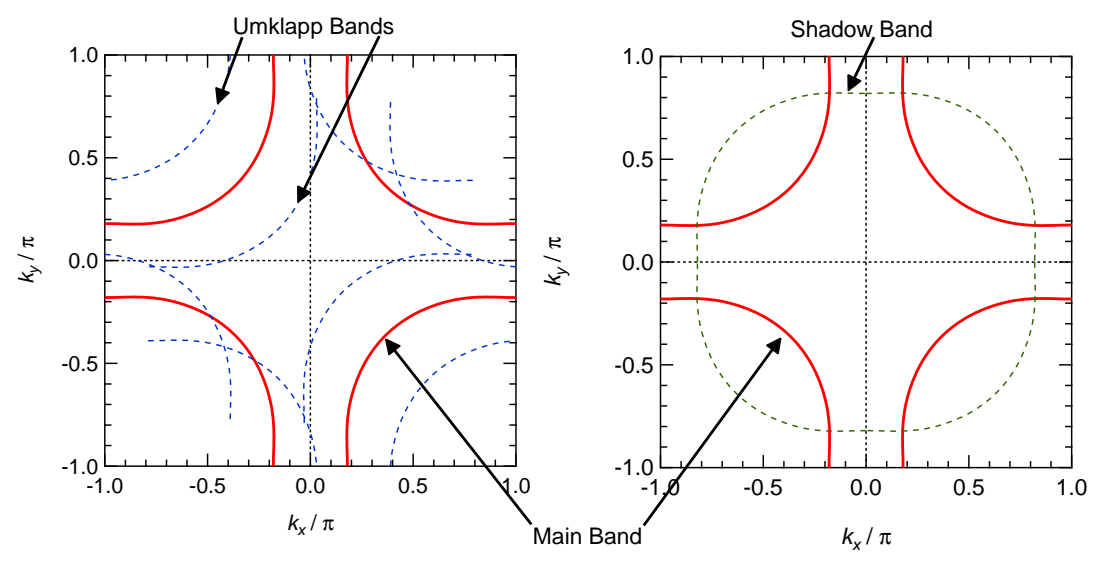

Figure 4.6 FS of Bi2212 showing the main (red), umklapp (dashed blue), and shadow (dashed green) bands.

can be removed by adding lead to the samples which relax the Bi-O plane. The second band that is sometimes present in cuprates is the shadow band, Figure 4.6 right in dashed green. The origins of the shadow bands are still not completely clear. It either comes from the antiferromagnetic correlations or from the structural distortion in the sample; but the research is pointing towards structural distortion[84]. 

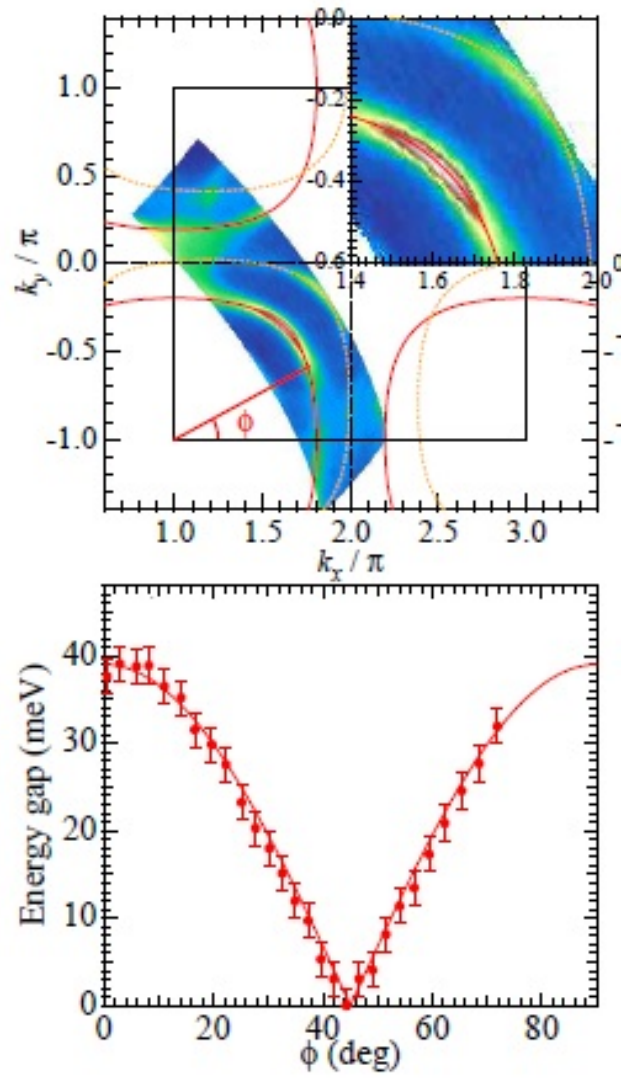

Figure 4.7 Top - Fermi surface map of optimally-doped Bi2212 taken in the second zone. In the color plot red corresponds to a high photoelectron count and blue to a lower photoelectron count, the red curve is the tight binding fit of the main band, and the yellow dotted curve is the tight binding fit of the shadow band. The upper right hand corner is a magnified view of the FS. Bottom - the size of the gap as a function of angle around the FS. 


\section{Fermi surface gaps}
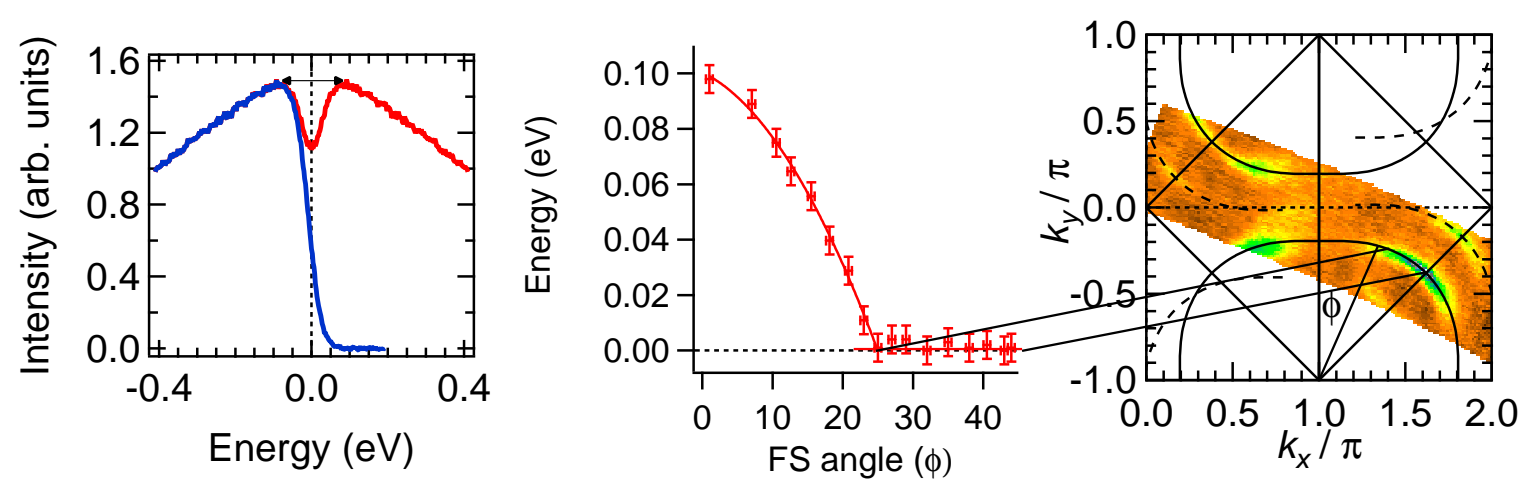

Figure 4.8 Pseudogap as a function of angle on the main band of UD75K Bi2212 at $\mathrm{T}=140 \mathrm{~K}$. Moving left to right: EDC at $k_{F}$ blue, symmetrized EDC $k_{F}$ red taken at $(\pi, 0)$; gap size as a function of angle(defined in far right figure); FS integrating within \pm $10 \mathrm{meV}$ of $\mathrm{E}_{F}$, the black line from the middle figure show the portion of the FS that is not gapped, e.g., white, blue, and green FS (25-45 degrees), this region is called the Fermi Arc.

The size and symmetry of the superconducting gap and pseudogap are probably the two most important properties of cuprates studied by ARPES. The consensus in the field is that the superconducting order parameter in cuprates has a $d$-wave symmetry with a node in the gap function along the $(\pi, \pi)$ direction. An example of the $d$-wave gap symmetry is shown in Figure 4.7. The gap in the FS is a maximum at $\phi=0$ and goes to zero at $\phi=45$. This creates four nodes/points on the FS. This is in contrast to the symmetry of the pseudogap, which closes before the node (Figure 4.8 middle). The magnitude of the gap is commonly found by symmetrizing the EDC at $k_{F}$ (Figure 4.8 - left red curve) and fitting to a symmetrized Dynes function [85]. One very interesting feature at the FS, due to the pseudogap, is called the Fermi arc. The arc is present in the pseudogap state, where the gap function goes to zero, 25-45 degrees in Figure 4.8 - right. This non-gapped portion of the FS creates an arc when looking at the FS. The length of the arc is thought to change with doping, becoming shorter with lower doping (i.e. a longer portion of the FS is gapped) [86]. Chapter 7 will revisit this claim, as 

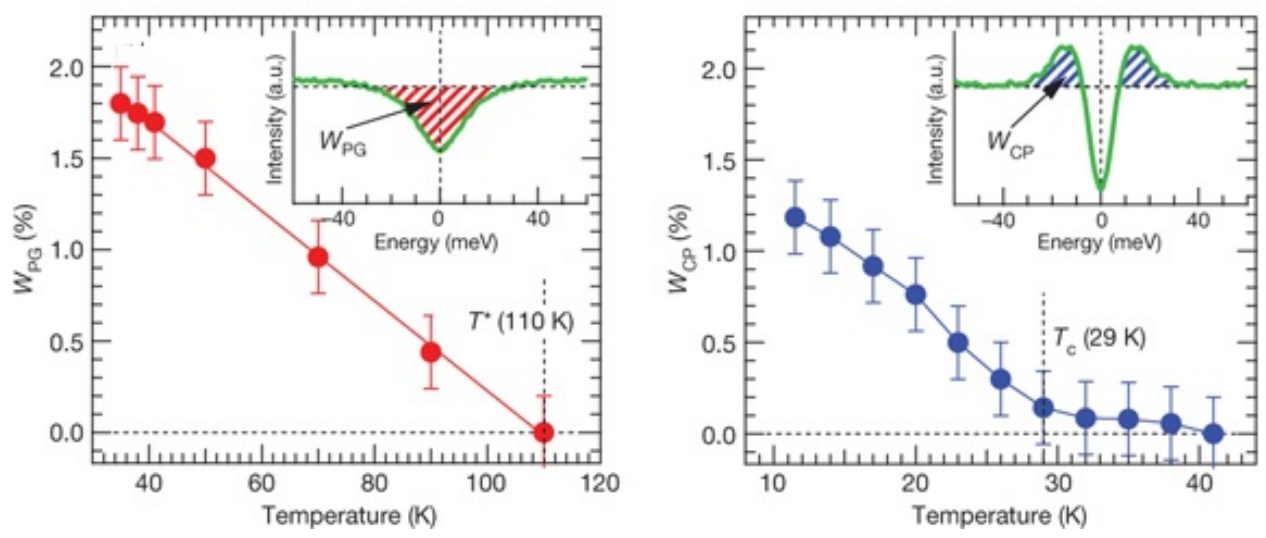

Figure 4.9 Left-pseudogap weight $\left(\mathrm{W}_{P G}\right)$ as a function of temperature on OD29K, the inserter shows a pictorial definition of the $\mathrm{W}_{P G}$. Right-coherent peak spectral weight $\left(\mathrm{W}_{C P}\right)$ as a function of temperature on OD29K, the inserter shows a pictorial definition of the $\mathrm{W}_{C P}$ adapted from Kondo et al. [110].

this may be only valid in the under doped side of the phase diagram.

There is a debate in the field of cuprates over the origins of the pseudogap and how it relates to superconductivity. Some people think the pseudogap is a preformed pair state where Cooper pairs form above $T_{c}$, but there is no long range coherence between the pairs [8797]. Others think the pseudogap competes with superconductivity by reducing the number of electrons that are available for superconductivity [98-110]. The next couple of paragraphs presents previous work done by the Ames laboratory ARPES group to show the pseudogap probably competes with superconductivity.

In 2009, Kondo et al. found a way to decouple the spectral components of the superconductivity and the pseudogap in Bi2201 over a wide range of dopings [110]. This is important because below $T_{c}$ the two spectral signature are convoluted together, making a direct comparison almost impossible. They decoupled the two spectral components by scanning the FS in the normal state (above $T^{*}$ ), in the pseudogap state (above $T_{c}$ but below $T^{*}$ ), and in the combined superconducting/pseudogap state (below $T_{c}$ ). By subtracting the normal state EDCs at $k_{F}$ from the pseudogap state they were left with a signature of the pseudogap which they called pseudogap spectral weight or $\mathrm{W}_{P G}$ (Figure 4.9 - left). By subtracting the pseudogap 


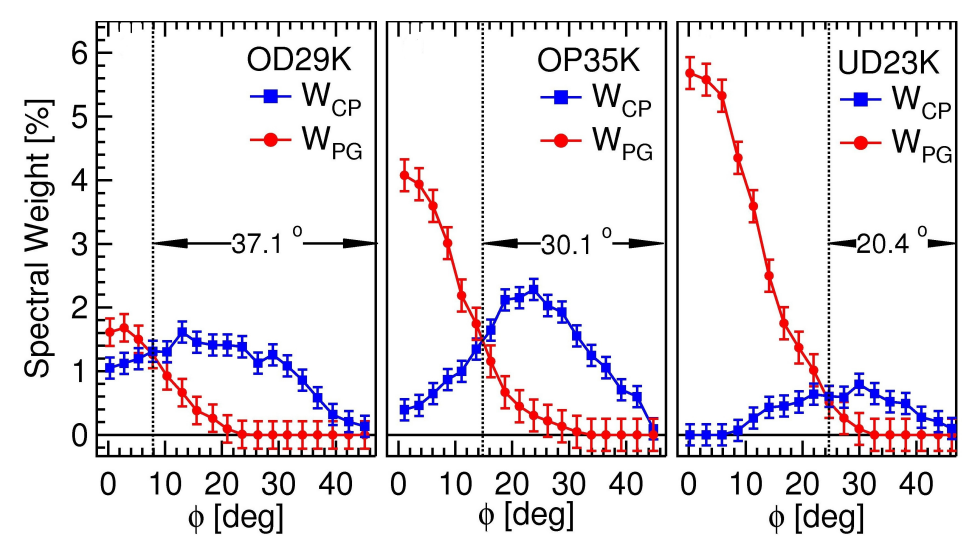

Figure 4.10 Momentum dependence of the spectral weight as a percent of the total spectrum of Bi2201 at three different dopings; left - over-doped $T_{c}=29 \mathrm{~K}$, middle - optimally-doped $T_{c}=35 \mathrm{~K}$, and right - under-doped $T_{c}=23 \mathrm{~K}$. The red circles represent percent of the $\mathrm{W}_{P G}$ and the blue $\mathrm{W}_{C P}$. The vertical dotted line show the crossover angle between $\mathrm{W}_{P G}$ and $\mathrm{W}_{C P}$. Figure adapted from Kondo et al. [110].

state data from the superconducting state EDCs at $k_{F}$ they were left with a signature of the superconducting state which they called the coherent peak spectral weight or $\mathrm{W}_{C P}$ (Figure 4.9 - right).

After decoupling the two weights $\left(\mathrm{W}_{P G}\right.$ and $\left.\mathrm{W}_{C P}\right)$, they compared the percent of the total spectrum associated with each as a function of angle (defined in Figure 4.7) along the FS. The data is presented in Figure 4.10; moving from left to right, over-doped $T_{c}=29 \mathrm{~K}$, optimallydoped $T_{c}=35 \mathrm{~K}$, and under-doped $T_{c}=23 \mathrm{~K}$ are shown. The blue squares are the percent of $\mathrm{W}_{C P}$ and the red circle are the percent of the $\mathrm{W}_{P G}$. The vertical dotted line is the crossover angle where the percent of the $\mathrm{W}_{P G}$ overtakes the percent of $\mathrm{W}_{C P}$. The data clearly shows the percent of the spectrum associated with the pseudogap gets larger when the percent of the spectrum associated with the superconductivity gets smaller. This anti-correlation between the two weights is opposite to what one would expect if they had the same origins. They concluded that the pseudogap formation from a preformed Cooper pairs state is clearly inconsistent with their results.

Right before the publication of this dissertation Kondo et al. [111] published another paper 
showing that there is indeed a preformed pair state above $T_{c}$ but it is below $T^{*}$ at $\mathrm{T}_{\text {pair }}(\sim 140$ K) and not related to the pseudogap. This third state matches previous studies of the Nernst

effect, NMR, and specific heat seeing superconductivity above $T_{c}$ which caused many people to think the pseudogap is a preformed pair state.

\section{Bibliography}

[60] H. K. Onnes. Communications from the Physical Laboratory of the University of Leiden (1911).

[61] W. Meissner and R. Ochsenfeld, Naturwissenschaften, 21, 787 (1933).

[62] A. M. Forrest, Eur. J. Phys. 4, 117 (1983).

[63] F. London and H. London. Proc. Roy. Soc. A149, 71 (1935).

[64] V. L. Ginzburg and L. D. Landau. Zh. Eksperim. i Teor. Fiz. 20, 1064 (1950).

[65] L. N. Cooper, Phys. Rev. 104, 1189 (1956).

[66] J. Bardeen, L. N. Cooper, and J. R. Schrieffer, Phys. Rev. 106, 162 (1957).

[67] J. Bardeen, L. N. Cooper, and J. R. Schrieffer, Phys. Rev. 108, 1175 (1957).

[68] J. G. Bednorz and K. A. Müller, Z. Phys. 64, 189 (1986).

[69] V. L. Ginzburg, Usp. Fiz. Nauk 161, 1 (1991).

[70] L. Gao, Y. Y. Xue, F. Chen, Q. Xiong, R. L. Meng, D. Ramirez, C. W. Chu, J. H. Eggert, and H. K. Mao, Phys. Rev. B 50, 4260 (2006).

[71] J. Nagamatsu, N. Nakagawa, T. Muranaka, Y. Zenitani, and J. Akimitsu, Nature 410, 63 (2001).

[72] Y. Kamihara, T. Watanabe, M. Hirano, and H. Hosono, J. Am. Chem. Soc. 130, 3296 (2008). 
[73] Ren Zhi-An, Lu Wei, Yang Jie, Yi Wei, Shen Xiao-Li, Zheng-Cai, Che Guang-Can, Dong Xiao-Li, Sun Li-Ling, Zhou Fang and Zhao Zhong-Xian, Chinese Phys. Lett. 25, 2215 (2008).

[74] Marianne Rotter, Marcus Tegel, and Dirk Johrendt, Phys. Rev. Lett. 101, 107006 (2008).

[75] Ni Ni, Iowa State University, Dissertation AAT 3389132 (2009).

[76] Kiyohisa Tanaka, Photoemission study of Bi-cuprate high-Tc superconductors in the lightly-doped to underdoped regions, University of Tokyo, Thesis (2004).

[77] E. Maxwell, Phys. Rev. 78, 477 (1950).

[78] K. Momma and F. Izumi, J. Appl. Crystallogr. 41, 653 (2008).

[79] H. Shaked, P. M. Keane, J.C. Rodriguez, F. F. Owen, R. L. Hitterman, and J. D. Jorgensen, Crystal Structures of the High-Tc Superconducting Copper-Oxides, Elsevier Science B.V., Amsterdam (1994).

[80] A. Tavana and M. Akhavan, Eur. Phys. J. B 73, 79 (2010).

[81] T. Kondo, R. Khasanov, J. Karpinski, S. M. Kazakov, N. D. Zhigadlo, T. Ohta, H. M. Fretwell, A. D. Palczewski, J. D. Koll, J. Mesot, E. Rotenberg, H. Keller, and A. Kaminski, Phys. Rev. Lett. 98, 157002 (2007).

[82] Andreas Erb and Michael Lambacher, Sampling the phase diagram of the cuprate superconductors, DFG (Deutsche Forschungsgemeinschaft) report FOR 538, http://www.wmi.badw-muenchen.de/FG538/projects/P4_crystal_growth/files/Erb_Crystals_2006.pdf (2006).

[83] Tsunehiro Takeuchi, Takeshi Kondo, Takio Kitao, Hiroyuki Kaga, Hongbo Yang, Hong Ding, Adam Kaminski, and Juan Carlos Campuzano, Phys Rev. Lett. 95, 227004 (2005).

[84] K. Nakayama, T. Sato, T. Dobashi, T. Takahashi, T. Kondo, T. Takeuchi, K. Kudo, and N. Kobayashi, Physica C 463-465, 48 (2007). 
[85] D. V. Evtushinsky, D. S. Inosov, V. B. Zabolotnyy, M. S. Viazovska, R. Khasanov, A. Amato, H.-H. Klauss, H. Luetkens, Ch. Niedermayer, G. L. Sun, V. Hinkov, C. T. Lin, A. Varykhalov, A. Koitzsch, M. Knupfer, B. Bchner, A. A. Kordyuk, and S. V. Borisenko, New Journal of Physics 11, 055069 (2009).

[86] Kiyohisa Tanaka, W. S. Lee, D. H. Lu, A. Fujimori, T. Fujii, Risdiana, I. Terasaki, D. J. Scalapino, T. P. Devereaux, Z. Hussain, and Z.-X. Shen, Science 314, 1910 (2006).

[87] H. Alloul, A. Mahajan, H. Casalta, and O. Klein, Phys. Rev. Lett. 70, 1171 (1993).

[88] M. R. Norman, D. Pines, and C. Kallin, Adv. Phys. 54, 715 (2005).

[89] Y. Wang, L. Li, and N. P. Ong, Phys. Rev. B 73, 024510 (2006).

[90] A. Kanigel, M. R. Norman, M. Randeria, U. Chatterjee, S. Souma, A. Kaminski, H. M. Fretwell, S. Rosenkranz, M. Shi, T. Sato, T. Takahashi, Z. Z. Li, H. Raffy, K. Kadowaki, D. Hinks, L. Ozyuzer, and J. C. Campuzano, Nature Physics 2, 447 (2006).

[91] O. Fischer, M. Kugler, I. Maggio-Aprile, and C. Berthod, Rev. Mod. Phys. 79, 353 (2007).

[92] A. Kanigel, U. Chatterjee, M. Randeria, M. R. Norman, S. Souma, M. Shi, Z. Z. Li, H. Raffy, and J. C. Campuzano, Phys. Rev. Lett. 99, 157001 (2007).

[93] A. Kanigel, U. Chatterjee, M. Randeria, M. R. Norman, G. Koren, K. Kadowaki, and J. C. Campuzano, Phys. Rev. Lett. 101, 137002 (2008).

[94] H.-B. Yang, J. D. Rameau, P. D. Johnson, T. Valla, A. Tsvelik, and G. D. Gu, Nature 456, 77 (2008).

[95] M. Shi, A. Bendounan, E. Razzoli, S. Rosenkranz, M. R. Norman, J. C. Campuzano, J. Chang, M. Mnsson, Y. Sassa, T. Claesson, O. Tjernberg, L. Patthey, N. Momono, M. Oda, M. Ido, S. Guerrero, C. Mudry, and J. Mesot, EPL 88, 27008 (2009).

[96] M. R. Norman, Science 325, 1080 (2010). 
[97] M. R. Norman, A. Kanigel, M. Randeria, U. Chatterjee, and J. C. Campuzano, Phys. Rev. B 76, 174501 (2007).

[98] Kiyohisa Tanaka, W. S. Lee, D. H. Lu, A. Fujimori, T. Fujii, Risdiana, I. Terasaki, D. J. Scalapino, T. P. Devereaux, Z. Hussain, and Z.-X. Shen, Science 314, 1910 (2006).

[99] M. Le Tacon, A. Sacuto, A. Georges, G. Kotliar, Y. Gallais, D. Colson, and A. Forget, Nature Physics 2, 537 (2006).

[100] M. C. Boyer, W. D. Wise, Kamalesh Chatterjee, Ming Yi, Takeshi Kondo, T. Takeuchi, H. Ikuta, and E. W. Hudson, Nature Phys. 3, 802 (2007).

[101] W. S. Lee, I. M. Vishik, K. Tanaka, D. H. Lu, T. Sasagawa, N. Nagaosa, T. P. Devereaux, Z. Hussain, and Z. -X. Shen, Nature 450, 81 (2007).

[102] Takeshi Kondo, Tsunehiro Takeuchi, Adam Kaminski, Syunsuke Tsuda, and Shik Shin, Phys. Rev. Lett. 98, 267004 (2007).

[103] Li Yu, D. Munzar, A. V. Boris, P. Yordanov, J. Chaloupka, Th. Wolf, C. T. Lin, B. Keimer, and C. Bernhard, Phys. Rev. Lett. 100, 177004 (2008).

[104] H. A. Mook, Y. Sidis, B. Fauqu, V. Baldent, and P. Bourges, Phys. Rev. B 78, 020506(R) (2008).

[105] Y. H. Liu, Y. Toda, K. Shimatake, N. Momono, M. Oda, and M. Ido, Phys. Rev. Lett. 101, 137003 (2008).

[106] R. Khasanov, Takeshi Kondo, S. Strüssle, D. O. G. Heron, A. Kaminski, H. Keller, S. L. Lee, and Tsunehiro Takeuchi, Phys. Rev. Lett. 101, 227002 (2008).

[107] J.-H. Ma, Z.-H. Pan, F. C. Niestemski, M. Neupane, Y.-M. Xu, P. Richard, K. Nakayama, T. Sato, T. Takahashi, H.-Q. Luo, L. Fang, H.-H. Wen, Ziqiang Wang, H. Ding, and V. Madhavan, Phys. Rev. Lett. 101, 207002 (2008). 
[108] T. Yoshida, M. Hashimoto, S. Ideta, A. Fujimori, K. Tanaka, N. Mannella, Z. Hussain, Z.-X. Shen, M. Kubota, K. Ono, Seiki Komiya, Yoichi Ando, H. Eisaki, and S. Uchida, Phys. Rev. Lett. 103, 037004 (2009).

[109] Y. Kohsaka, C. Taylor, P. Wahl, A. Schmidt, Jhinhwan Lee, K. Fujita, J. W. Alldredge, K. McElroy, Jinho Lee, H. Eisaki, S. Uchida, D.-H. Lee and J. C. Davis, Nature 454, 1072 (2008).

[110] Takeshi Kondo, Rustem Khasanov, Tsunehiro Takeuchi, Jorg Schmalian, and Adam Kaminski, Nature 457, 296 (2009).

[111] Takeshi Kondo, Yoichiro Hamaya, Ari D. Palczewski, Tsunehiro Takeuchi, J. S. Wen, Z. J. Xu, Genda Gu, Joerg Schmalian, and Adam Kaminski, arXiv:1005.5309v1, accepted Nature Physics (2010). 


\title{
CHAPTER 5. ORIGINS OF LARGE CRITICAL TEMPERATURE VARIATIONS IN SINGLE-LAYER CUPRATES
}

A paper published in the Physical Review B: Phys. Rev. B 78, 054523 (2008)

A. D. Palczewski ${ }^{1,2}$, T. Kondo ${ }^{1,2}$, R. Khasanov ${ }^{3}$, N. N. Kolesnikov ${ }^{4}$, A. V. Timonina ${ }^{4}$,

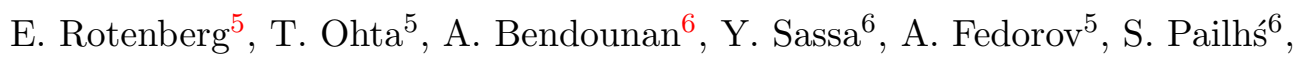
A. F. Santander-Syro ${ }^{7,8}$, J. Chang ${ }^{6}$, M. Shi ${ }^{9}$, J. Mesot $^{6}$, H. M. Fretwell ${ }^{1}$, and A. Kaminski ${ }^{1,2}$

\begin{abstract}
We study the electronic structures of two single layer superconducting cuprates, $\mathrm{Tl}_{2} \mathrm{Ba}_{2} \mathrm{CuO}_{6+\delta}$ (Tl2201) and $\left(\mathrm{Bi}_{1.35} \mathrm{~Pb}_{0.85}\right)\left(\mathrm{Sr}_{1.47} \mathrm{La}_{0.38}\right) \mathrm{CuO}_{6+\delta}(\mathrm{Bi} 2201)$ which have very different maximum critical temperatures (95 K and $35 \mathrm{~K}$ respectively) using Angular Resolved Photoemission Spectroscopy (ARPES). We are able to identify two main differences in their electronic properties. First, the shadow band that is present in double layer and low $T_{c, \max }$ single layer cuprates is absent in Tl2201. Recent studies have linked the shadow band to structural distortions in the lattice and the absence of these in Tl2201 may be a contributing factor in its $T_{c, \max }$. Second, Tl2201's Fermi surface (FS) contains long straight parallel regions near the antinode, while in Bi2201 the antinodal region is much more rounded. Since the size of the superconducting gap

\footnotetext{
${ }^{1}$ Division of Materials Science and Engineering, Ames Laboratory, Ames, Iowa 50011, USA

${ }^{2}$ Department of Physics and Astronomy, Iowa State University, Ames, Iowa 50011, USA

${ }^{3}$ Laboratory for Muon Spin Spectroscopy, Paul Scherrer Institut, CH-5232 Villigen PSI, Switzerland

${ }^{4}$ Institute of Solid State Physics, Chernogolovka, 142432 Russia

${ }^{5}$ Advanced Light Source, Berkeley National Laboratory, Berkeley, California 94720, USA

${ }^{6}$ Laboratory for Neutron Scattering, ETH Zurich and Paul Scherrer Institute, CH-5232 Villigen PSI, Switzerland

${ }^{7}$ Laboratoire Photons Et Matiére, UPR-5 CNRS, ESPCI, 10 rue Vauquelin, 75231 Paris cedex 5, France

${ }^{8}$ Labratoire de Physique des Solides, UMR-8502 CNRS, Universite Paris-Sud 11, Btiment 510 Orsay, 91405 France

${ }^{9}$ Swiss Light Source, Paul Scherrer Institute, CH-5232 Villigen PSI, Switzerland
} 
is largest in the antinodal region, differences in the band dispersion at the antinode may play a significant role in the pairing and therefore affect the maximum transition temperature.

\section{Introduction}

Despite more than 20 years of effort, there is still no consensus on what is the nature of the superconducting coupling mechanism in the high $T_{c}$ superconductors. Early theoretical works [112] proposed that interlayer interactions between the copper oxygen $(\mathrm{Cu}-\mathrm{O})$ planes in these quasi 2D materials played a key role in the pairing mechanism. However, some predictions from this model were later found to be inconsistent with experiment [113]. Yet, there remains empirical evidence that both the maximum transition temperature $\left(T_{c, \max }\right)$ and the size of the superconducting gap of the high temperature superconducting cuprates (HTSC) depends, sometimes strongly, on the number of $\mathrm{Cu}-\mathrm{O}$ layers per unit cell [114]. Bismuth [115], thallium [116], and mercury [117] - based cuprates all show an increase in $T_{c, \max }$ with the number of $\mathrm{Cu}^{-}$ $\mathrm{O}$ layers. While $T_{c, \max }$ increases with the number of $\mathrm{Cu}-\mathrm{O}$ layers (peaking at 3 layers per unit cell), it is not always the same for a given number of layers. In particular, there are two single layer materials, $\mathrm{Tl}_{2} \mathrm{Ba}_{2} \mathrm{CuO}_{6+\delta}(\mathrm{Tl2201})$ [118] and $\mathrm{HgBa}_{2} \mathrm{CuO}_{4+\delta}(\mathrm{Hg} 1201)$ [119] $\left(T_{c, \text { max }} \sim 95\right.$ $\mathrm{K}$ ), whose transition temperatures are actually closer to that of other double layer cuprates. This could mean that either $T_{c, \max }$ is somehow enhanced in Tl2201 and $\operatorname{Hg} 1201$ or that $T_{c, \max }$ for all single layer cuprates is intrinsically closer to $95 \mathrm{~K}$ and other mechanisms, for example, lattice distortions in the Bismuth-based materials [120] reduce $T_{c, \max }$. One can imagine that adding more $\mathrm{Cu}-\mathrm{O}$ layers per unit cell to the $\mathrm{Bi}-$ based material (going from Bi2201 to Bi2212) creates an additional pairing channel, thereby enhancing the superconductivity and pushing the $T_{c, \max }$ back up to $\sim 95 \mathrm{~K}$. To help explore these ideas and explain the large variation of $T_{c, \max }$ of the single layer compounds, it is essential to look for differences in their electronic structure through Angle-Resolved Photoemission Spectroscopy (ARPES) [121, 122].

Here we report an ARPES study on the electronic structure of two single layer cuprates with distinctly different maximum critical temperatures: $\mathrm{Tl}_{2} \mathrm{Ba}_{2} \mathrm{CuO}_{6+\delta}(\mathrm{Tl} 2201) T_{c} \sim 95 \mathrm{~K}$ and $\left(\mathrm{Bi}_{1.35} \mathrm{~Pb}_{0.85}\right)\left(\mathrm{Sr}_{1.47} \mathrm{La}_{0.38}\right) \mathrm{CuO}_{6+\delta}(\mathrm{Bi} 2201) T_{c} \sim 35 \mathrm{~K}$. We find two striking differences 
in the Fermi surface (FS) maps at the chemical potential. First, the shadow band (usually attributed to structural distortions $[120,123,124]$ ) is present in single layer Bi2201 (and double layer Bi2212) but is absent in Tl2201. Second (and possibly more important), the FS of Tl2201 has long parallel "nested" regions close to the antinodes (where the superconducting gap reaches its maximum value). This feature is very similar to that found in double layered Bi2212 with a $T_{c, \max }$ of $\sim 95 \mathrm{~K}$, while it is absent in Bi2201. In other words, materials with a high $T_{c}$ have strongly nested FS.

\section{Experimental Details}

Optimally doped Bi2201 single crystals were grown using the floating zone (FZ) method [125]. The substitution of $\mathrm{Pb}$ suppresses the modulation in the $\mathrm{Bi}-\mathrm{O}$ layers [126] that normally causes complications (superlattice) in interpreting the band structure in pristine $\mathrm{Bi}_{2} \mathrm{Sr}_{2} \mathrm{CuO}_{6+\delta}$ $[121,122]$. Near optimally doped $\mathrm{Tl}_{2} \mathrm{Ba}_{2} \mathrm{CuO}_{6+\delta}$ crystals were grown in an air atmosphere inside zirconium dioxide multilayered crucibles [127, 128]. Single crystals samples of both materials used in ARPES experiments are of exceptional quality as evidenced by very sharp superconducting transitions with typical widths $\sim 2-4 \mathrm{~K}$ shown in [Figure 5.1(b)-(c)]. FS measurements for Tl2201 were performed at the Swiss Light Source (SLS) on beamline X09LAHRPES with a Scienta SES2002 analyzer at $49 \mathrm{eV}$ photon energy. The choice of photon energy was dictated by need to maximize both the signal intensity and energy resolution. As evident from Figure 5.5 there are two main energy for which the signal reaches maximum: $49 \mathrm{eV}$ and $74 \mathrm{eV}$. The signal is certainly stronger when using the latter, however due to characteristics of beamline the energy resolution there would be significantly reduced. The energy and angular resolution was set to $30 \mathrm{meV}$ and $0.5^{\circ}$ respectively. Electronic structure information for Bi2201 and Tl2201 was acquired at the Advanced Light source (ALS) on Beamline 7.0.1 with the SCIENTA R4000 analyzer at $105 \mathrm{eV}$ photon energy. The energy and angular resolution of the $\mathrm{R} 4000$ was set to $40 \mathrm{meV}$ and $0.5^{\circ}$ respectively. Tl2201 photon energy dependence data was taken at the ALS on beamline 12.0.1.1 using a SCIENTA 100 analyzer. The energy and angular resolutions was set to $50 \mathrm{meV}$ and $0.3^{\circ}$ respectively. Bi2201 doping dependence data 
was acquired on a Scienta SES2002 analyzer using a Gammadata VUV5000 photon source $(\mathrm{HeI} \alpha)$ at Iowa State University. The energy and angular resolution was set to $5 \mathrm{meV}$ and $0.13^{\circ}$ respectively. All data was acquired on in situ cleaved crystals at or below $20 \mathrm{~K}$ under UHV, with the samples being kept at their cleaving temperature throughout the measurement process. During the measurement process we had to cleave multiple Tl2201 samples in order to get reliable and reproducible results. This was mainly due to Tl2201's inability to cleave nicely. Bi2201 on the other hand almost always cleaves nicely, so multiple cleaves were not as important.

\section{Data and Analysis}

The schematic crystal structure of Tl2201 and Bi2201 are shown in Figure 5.1 (a) [79]. Each material's unit cell contains a single $\mathrm{Cu}-\mathrm{O}$ layer with dual layers of $\mathrm{Tl}-\mathrm{O}$ and $\mathrm{Ba}-\mathrm{O}(\mathrm{Tl} 2201)$ or $\mathrm{Bi}-\mathrm{O}$ and $\mathrm{Sr}-\mathrm{O}$ (Bi2201). We note that Tl2201 has a tetragonal (i.e. $a=b$ ) structure with nearly perfectly flat $\mathrm{Cu}-\mathrm{O}$ layers and a slight buckling in the $\mathrm{Tl}-\mathrm{O}$ and $\mathrm{Ba}-\mathrm{O}$ layers [127]. In contrast, Bi2201's structure has a degree of orthorombicity (i.e. $a \cong b$ ) accompanied by buckling in all layers [118]. The two materials also have very different cleaving properties. Tl2201 has strong bonding between the layers, which makes it difficult to cleave, often leaving behind a rather rough surface, whereas, Bi2201 is very well known for excellent cleaving properties and is material of choice for surface studied such as ARPES or STM/STS. This is because the bonding between adjacent $\mathrm{Bi}-\mathrm{O}$ layers is due to Van der Waals interaction. In majority of cases after cleaving we were able to obtain a flat mirror-like surfaces.

The ARPES intensity integrated from $20 \mathrm{meV}$ to $-40 \mathrm{meV}$ about the chemical potential is plotted as a function of momentum for Bi2201 and Tl2201 in [Figure 5.2 (a) and (b)] respectively. The bright areas correspond to high intensity and represent the Fermi surface (FS) - those locations in momentum space where the band crosses the chemical potential. One can see that both FS are similar to the usual calculations of a $\mathrm{Cu}-\mathrm{O}$ layer inside a cuprate [130, 131], with a couple of distinct differences. First, the shadow band, found in some cuprates $[120,124,132]$ including single layer Bi2201 $\left(T_{c, \max }=35 \mathrm{~K}\right.$, left panel $)$ and LSCO $\left(T_{c, \max }=40\right.$ 
$\mathrm{K})$ as well as two layer $\operatorname{Bi2212}\left(T_{c, \text { max }} \cong 95 \mathrm{~K}\right)$, is absent in single layer Tl2201 $\left(T_{c, \text { max }} \cong 95 \mathrm{~K}\right.$, right panel). The second more subtle difference is the shape of the FS close to the antinode $(\pi, 0)$. To better compare the shape of the FS, we have performed a tight binding analysis on each of our samples. The results from these fits are shown in Figure 5.2 (c). The fitting analysis was performed using full 3D band dispersion data, examples of which are shown in Figure 5.3. We also present the published tight binding fits for Bi2212 [133] in Figure 5.2 (c) for comparison. Fitting parameters for all three cases are presented in Table 1.

Table 5.1 Tight Binding fitting function $(\varepsilon(\vec{k}))$ and experimental fit for Bi2201, Tl2201 and Bi2212 [133] where $\varepsilon(\vec{k})=\sum c_{i} \eta_{i}(\vec{k})$

\begin{tabular}{|c|ccc|}
\hline$\eta_{i}(\vec{k})$ & $c_{i} \mathrm{Bi} 2201$ & $c_{i}$ Tl2201 & $c_{i} \mathrm{Bi} 2212$ \\
\hline 1 & $0.16895 \pm 0.013$ & $0.24103 \pm 0.0202$ & 0.1305 \\
$\frac{1}{2}\left(\cos k_{x}+\cos k_{y}\right)$ & $-0.73338 \pm 0.0161$ & $-0.72153 \pm 0.0328$ & -0.5951 \\
$\cos k_{x} \times \cos k_{y}$ & $0.11389 \pm 0.00786$ & $0.14813 \pm 0.00935$ & 0.1636 \\
$\frac{1}{2}\left(\cos 2 k_{x}+\cos 2 k_{y}\right)$ & $-0.11086 \pm 0.00573$ & $-0.17287 \pm 0.0115$ & -0.0519 \\
$\frac{1}{2}\left(\cos 2 k_{x} \times \cos 2 k_{y}+\cos k_{x} \times \cos 2 k_{y}\right)$ & $-0.049688 \pm 0.0248$ & $-0.01604 \pm 0.0359$ & -0.1117 \\
$\cos 2 k_{x} \times \cos 2 k_{y}$ & $0.045032 \pm 0.00751$ & $0.048246 \pm 0.016$ & 0.051 \\
\hline
\end{tabular}




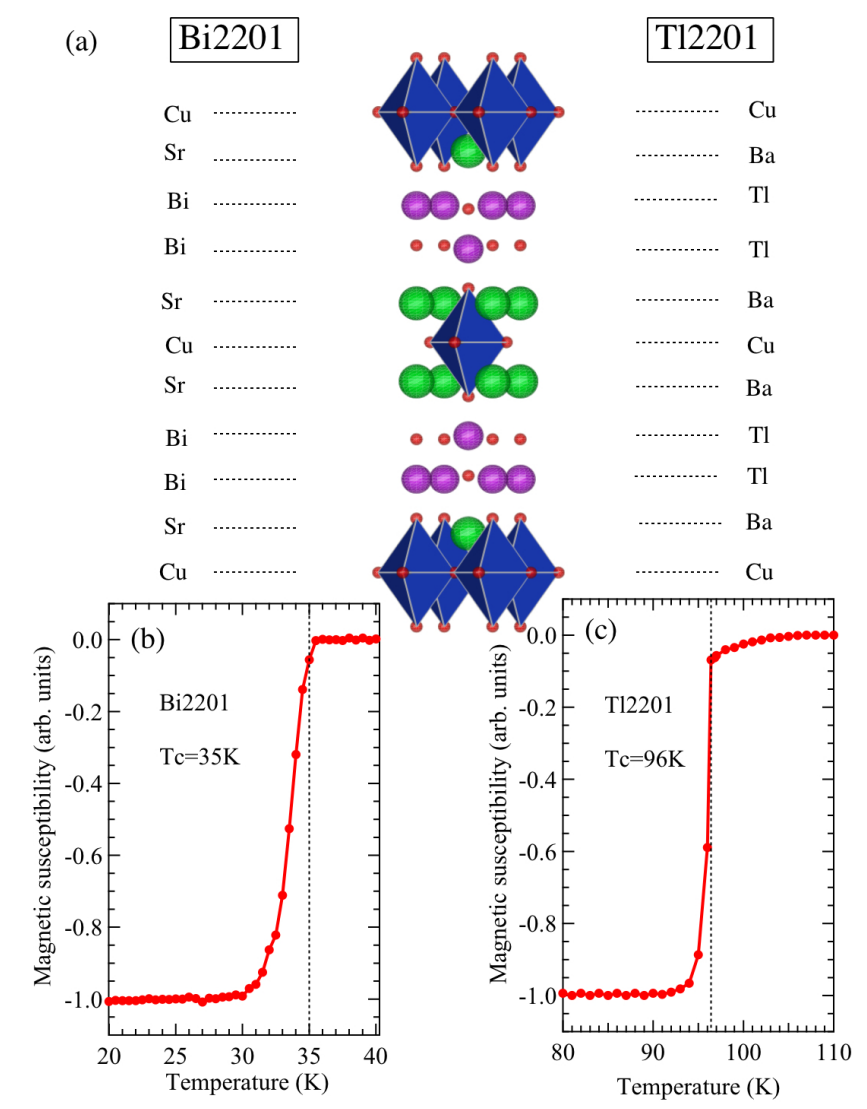

Figure 5.1 (a) Schematic structure of $\mathrm{Bi}_{2} \mathrm{Sr}_{2} \mathrm{CuO}_{6+\delta}$ (Bi2201) and $\mathrm{Tl}_{2} \mathrm{Ba}_{2} \mathrm{CuO}_{6+\delta}$ (Tl2201). The small red dots represent oxygen atoms and the other larger atoms are labeled by the symbol on the left (Bi2201) or right (Tl2201). Each layer is made up of the particular atoms bonded to oxygen, with the double pyramids representing copper oxygen bonds. (b)-(c) SQUID magnetization curves for Bi2201 and Tl2201. 

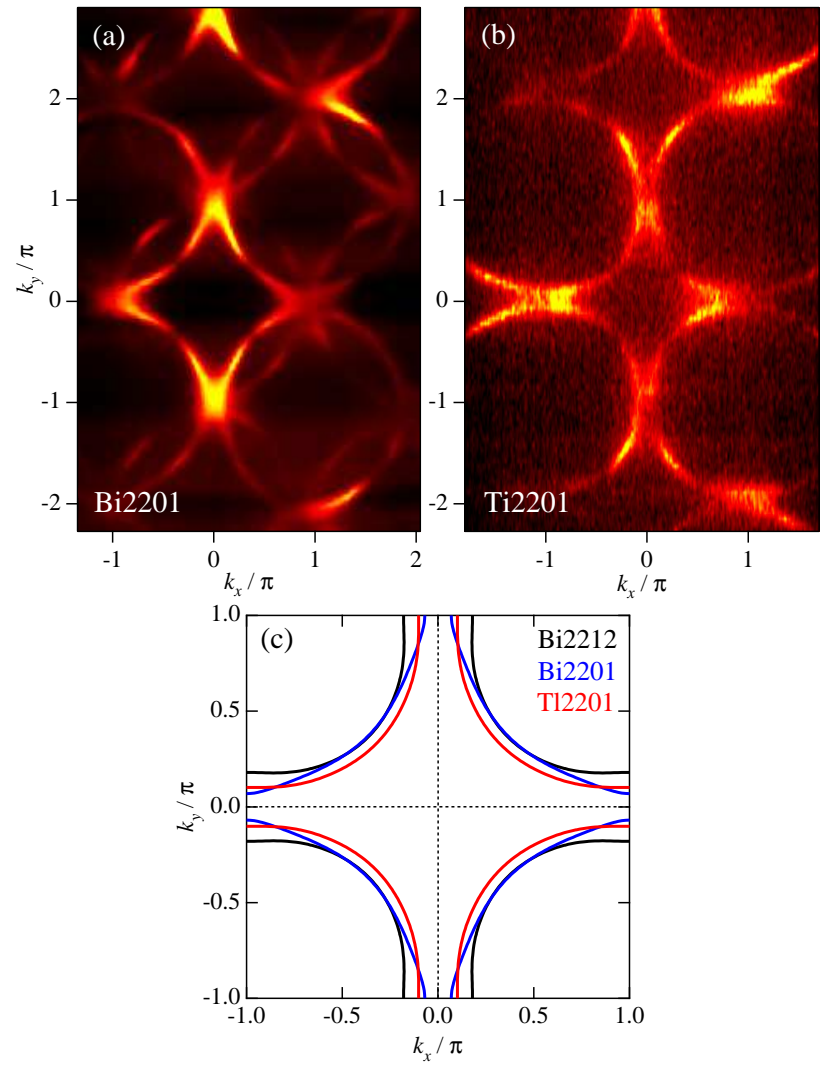

Figure 5.2 Intensity at the Fermi energy in multiple Brillouin zones for (a) Bi2201 and (b) Tl2201. All data was collected at a photon energy of $105 \mathrm{eV}$. High (low) intensity regions appear bright (dark) in the color map. (c) Tight binding fitting plots, Bi2212 [133] (black), Bi2201 (blue) and Tl2201 (red), fitting parameters for (c) are found in Table 1. 


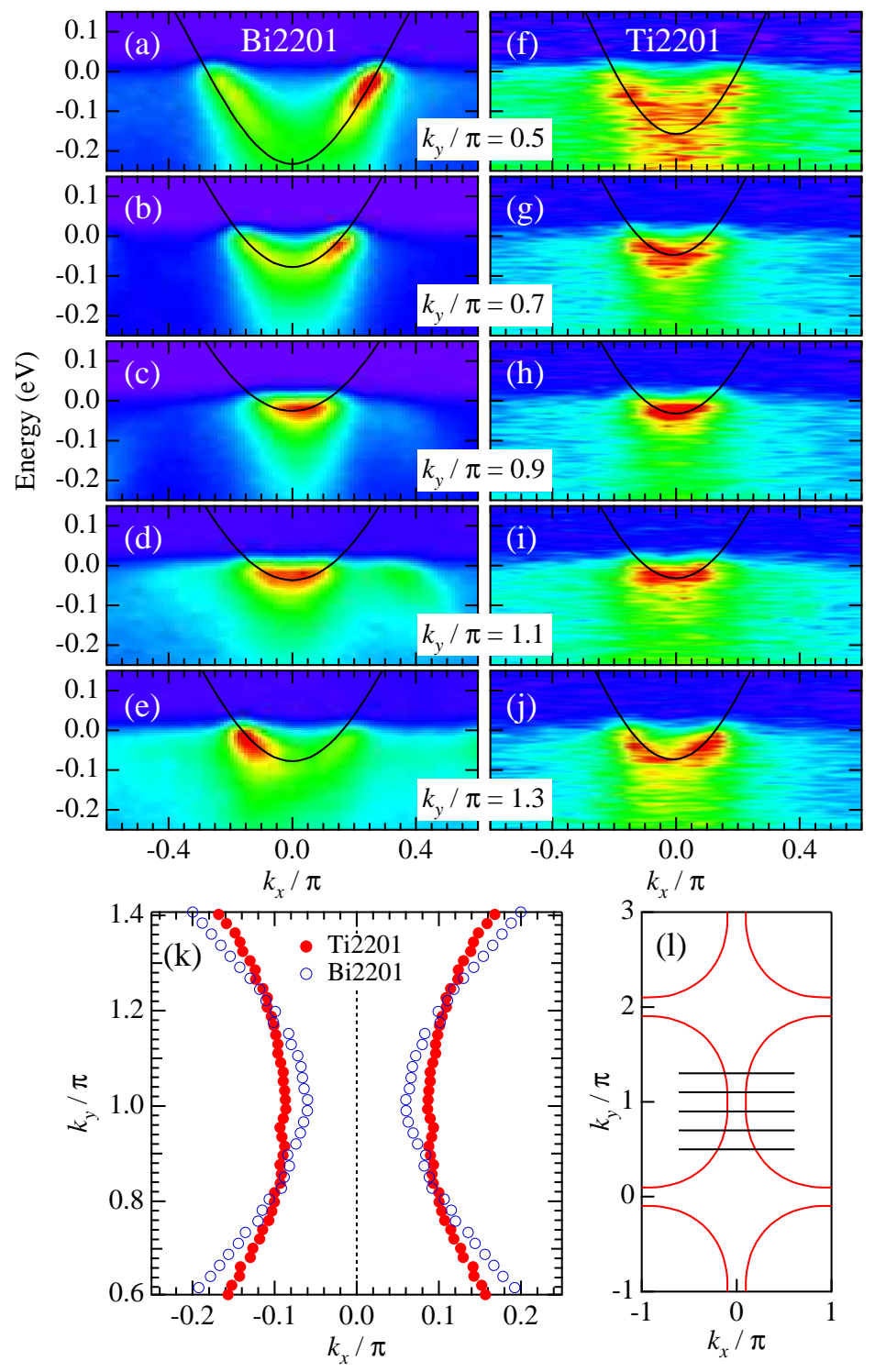

Figure 5.3 Momentum distribution curve for (a)-(e) Bi2201 and (f)-(j) Tl2201 taken at $k_{y} / \pi=0.5,0.7,0.9,1.1$ and 1.3 . The lowest intensity corresponds to red while the highest intensity corresponds to dark blue moving through the color spectrum. The colored pictures are the original ARPES data while the black lines are tight binding fit. The tight binding fitting parameters for the black lines are located in Table 1, (k) FS taken from peak position of MDC for Bi2201 (blue dots) and Tl2201 (red crosses), (l) schematic MDC location for (a)-(j). 
Based on these parameters we have calculated the carrier concentration level for the three systems: $0.17 \%$ for $\mathrm{Bi} 2212,0.27 \%$ for $\mathrm{Bi} 2201$ and $0.35 \%$ for Tl2201. The shape of the FS for Tl2201 and Bi2212 are almost identical; the only visual difference between the two arises from the differences in their carrier concentrations. They both display long, nearly parallel FS segments close to the antinode. The FS of Bi2201 is quite different in this region of momentum space. Bi2201 FS is much more rounded with no significant parallel segments. We have to point out that the length of the parallel segments in the antinodal regions will, in principle, depend on carrier concentration. In heavily overdoped cuprates, the antinodal regime of the FS can become less parallel and eventually close (disappearing completely from the FS) [134]. In our case, Tl2201 has a higher carrier concentration (more overdoped) than the Bi2201, yet Tl2201's antinodal FS nesting is still much greater than in Bi2201. To show that Bi2201's rounded FS is not a doping dependent feature but a fundamental characteristic, we present Figure 5.4.

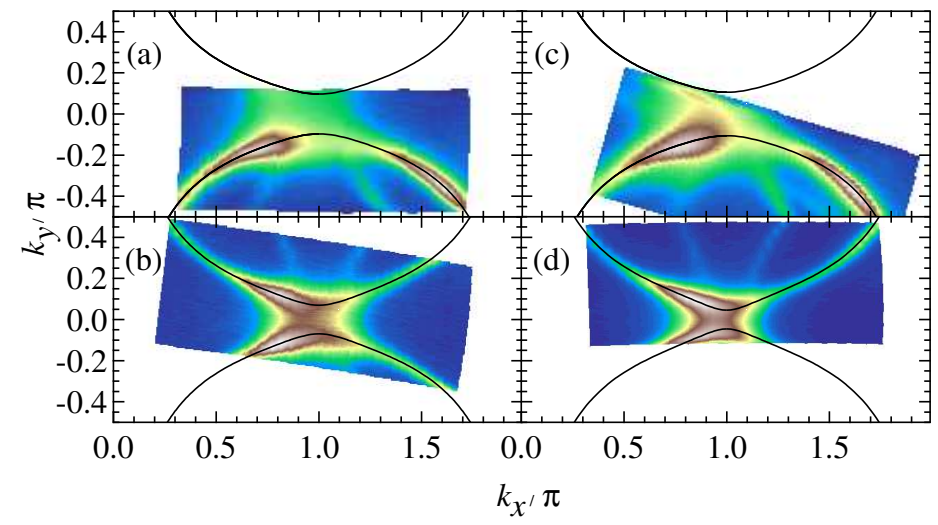

Figure 5.4 Intensity maps of Bi2201 taken around $(\pi, 0)$ for different carrier concentrations (a) $0.23 \%$, (b) $0.25 \%$, (c) $0.27 \%$, and (d) $0.29 \%$, with black line represents the tight binding fits for each doping level.

Moving from top to bottom and left to right, i.e. (a)-(d), we show the FS of Bi2201 about $(\pi, 0)$ at carrier concentration levels of $0.23 \%, 0.25 \%, 0.27 \%, 0.29 \%$ respectively. We see the shape changes slightly as we change doping, as is expected yet, the general roundness 
remained throughout all dopings levels. Figure 5.5 (a) shows the peak intensity verse photon energy for Tl2201 taken at a constant region of momentum space near $(\pi, 0)$. The variation in the intensity arises from the matrix element effect [135] during the photoemission process. [Figure $5.5(\mathrm{~b})-(\mathrm{d})]$ shows how the matrix elements can affect the overall dispersion with some energies being better than other for data acquisition. From this curve we have identified 49 $\mathrm{eV}$ and $74 \mathrm{eV}$ as the best energies for obtaining high resolution data from Tl2201 samples.
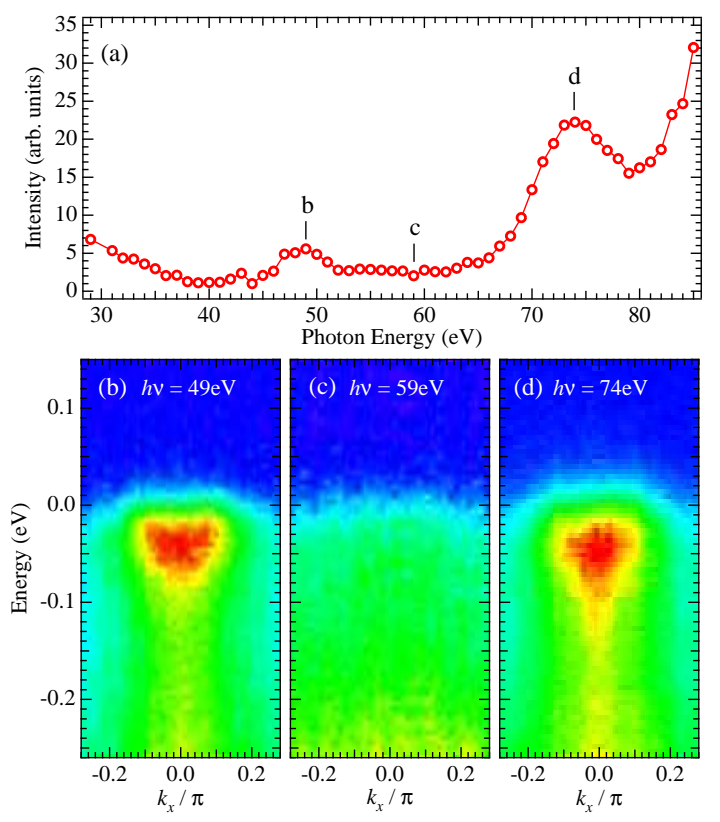

Figure 5.5 (a) Photon energy dependence for Tl2201 taken at around $(\pi, 0)$. Markers in (a) are at $49 \mathrm{eV}, 59 \mathrm{eV}$ and $74 \mathrm{eV}$ respectively, corresponding to the energy momentum cuts in (b), (c), and (d). In (b)-(d) dark blue corresponds to low intensity, while red corresponds to high intensity moving through the color spectrum.

\section{Discussion}

We now discuss why $T_{c, \text { max }}$ is much higher in Tl2201 compared to Bi2201. First, Tl2201 has a tetragonal crystal structure with flat $\mathrm{Cu}-\mathrm{O}$ layers, whereas $\mathrm{Bi} 2201$ is orthorhombic with buckled $\mathrm{Cu}-\mathrm{O}$ layers [118]. It is known that local lattice distortions (produced by chemical 
inhomogeneity) can reduce the value of $T_{c}$ in a systematic way [137]. It has also been shown that the larger the $\mathrm{Cu}-\mathrm{O}$ plane buckling angle, the lower the $T_{c, \max }$ [136]. So, distortions in the $\mathrm{Cu}-\mathrm{O}$ planes have long been known to cause a lowering of the $T_{c, \max }$. Second, our results show that the FS of Tl2201 does not contain a shadow band, but Bi2201 does. There are several explanations for the origins of the shadow band. The most convincing explanation to date is that it is due to structural distortions, either in the form of an orthorhombic distortion of the lattice [120], and/or by diffraction of the outgoing photoelectron by the superstructure of the $\mathrm{BiO}$ layer at the surface. Whatever the cause, if the shadow band is absent (as in Tl2201), it suggests that the material is free of the structural distortions that could potentially lower the $T_{c, \max }$ Finally, Tl2201 has strong interlayer interactions that are absent in Bi2201. The same strong interlayer bonding is also present in another high $T_{c, \max }$ single layer cuprate Hg1201 ( $\left.T_{c, \max } \sim 95 \mathrm{~K}\right)$ [119]. Given the above, our observation that Tl2201 does not exhibit a shadow band is fully consistent with the absence of structural distortions of its lattice and its unusually high $T_{c, \max }$. We now address the fact that $\mathrm{Bi} 2212$ is known to have buckled $\mathrm{Cu}-\mathrm{O}$ planes, orthorhombic distortions, a shadow band and weak interlayer interactions, yet it still has a high $T_{c, \max }$, which is comparable to that of $\mathrm{Tl} 2201$. We speculate that the extra $\mathrm{Cu}-\mathrm{O}$ layer per unit cell in Bi2212 enhances the superconductivity and raises the $T_{c, \max }$. This has been seen in other multilayered cuprates where Cooper pairs are allowed to tunnel between the $\mathrm{Cu}-\mathrm{O}$ layers through Josephson coupling, raising $T_{c, \max }[138,139]$.

Finally, our data shows a relationship between the length of the long parallel (nested) FS segments centered about $(\pi, 0)$ and $T_{c, \max }$. Looking back to Figure 5.2 (c) we see that Tl2201 and Bi2212 have very similar nested FS segments and approximately the same $T_{c, \max }$. In contrast, Bi2201's FS segments are much rounder with a lower $T_{c, \max }$. Our data suggests that FS nesting at the antinode is related to the enhanced $T_{c, \max }$. We also note that the superconducting and pseudo-gaps reach a maxima in these regions [140, 141], with other studies suggesting this region is critical in understanding how cuprate superconductivity works [142, 144-146]. Our observation of significant FS nesting in Tl2201 is an important new result. 


\section{Conclusions}

In conclusion, we report a comparative study on the electronic structures of two single layer cuprates T12201 $T_{c, \max } \sim 95 \mathrm{~K}$ and Bi2201 $T_{c, \max } \sim 35 \mathrm{~K}$, along with photon energy data for Tl2201. We find two striking differences in the occurrence of the shadow band and the shape of the FS close to the antinodes. First, the shadow band in single layer Bi2201 and double layer Bi2212 is absent in Tl2201. Second Tl2201 has long parallel (nested) regions on its FS (similar to double layer Bi2212 with $T_{c, \max } \sim 95 \mathrm{~K}$ ), while these regions are much smaller (if not absent) in low $T_{c, \max }$ Bi2201. Our data shows two non trivial results for superconducting cuprates. First, there may be a balance between structural distortions and interlayer interactions that help control $T_{c, \max }$ in the cuprates. Second, there is a qualitative relationship between the length of the antinodal nesting and $T_{c, \max }$ in our cuprates.

\section{Acknowledgments}

The work at the Ames Laboratory was supported by the Department of Energy at Iowa State University. Ames laboratory is supported under Contract No. DE-AC02-07CH11358. The Advanced Light Source is supported by the Director, Office of Science, Office of Basic En-

ergy Sciences, of the U.S. Department of Energy under Contract No. DE-AC02-05CH11231. This research project is also supported by the European Commission under the 6th Framework Programme: Strengthening the European Research Area, Research Infrastructures. Contract No: RII3-CT-2004-506008'.

\section{Bibliography}

[112] P.W. Anderson, The Theory of Superconductivity in the High-T $T_{c}$ Cuprate, Princeton Univ Press Princeton, NJ, (1997).

[113] A. A. Tsvetkov, D. van der Marel, K. A. Moler, J. R. Kirtley, J. L. de Boer, A. Meetsma, Z. F. Ren, N. Koleshnikov, D. Dulic, A. Damascelli, M. Gröninger, J. Schötzmann, J. W. van der Eb, H. S. Somal, and J. H. Wang, Nature 390, 360 (1998). 
[114] T. Sato, H. Matsui, S. Nishina, T. Takahashi, T. Fujii, T. Watanabe, and A. Matsuda, Phys. Rev. Lett. 89, 067005 (2002).

[115] D. L. Feng, A. Damascelli, K. M. Shen, N. Motoyama, D. H. Lu, H. Eisaki, K. Shimizu, J.-i. Shimoyama, K. Kishio, N. Kaneko, M. Greven, G. D. Gu, X. J. Zhou, C. Kim, F. Ronning, N. P. Armitage, and Z.-X Shen, Phys. Rev. Lett. 88, 107001 (2002).

[116] Y. C. Ma and N.L. Wang, Phys. Rev. B 72, 104518 (2005).

[117] C. Ambrosch-Draxl, E. Ya. Sherman, H. Auer, and T. Thonhauser, Phys. Rev. Lett. 92, 187004 (2004).

[118] C. C. Torardi, M. A. Subramanian, J. C. Calabrese, J. Gopalakrishnan, E. M. McCarron, K. J. Morrissey, T. R. Askew, R. B. Flippen, U. Chowdhry, and A. W. Sleight, Phys. Rev. B 38, 225 (1988).

[119] W. S. Lee, T. Yoshida, W. Meevasana, K. M. Shen, D. H. Lu, W. L Yang, X. J. Zhou, X. Zhao, G. Yu, Y. Cho, M. Greven, Z. Hussain, and Z.-X. Shen arXiv:cond-mat/0606347 v1 (2006).

[120] K. Nakayama, T. Sato, T. Dobashi, K. Terashima, S. Souma, H. Matsui, T. Takahashi, J. C. Campuzano, K. Kudo, T. Sasaki, N. Kobayashi, T. Kondo, T. Takeuchi, K. Kadowaki, M. Kofu, and K. Hirota, Phys. Rev. B 74, 054505 (2006).

[121] J. C. Campuzano, M. R. Norman, and M. Randeria, in The Physics of Superconductors, Vol. 2, ed. K. H. Bennemann and J. B. Ketterson, (Springer, Berlin), p. 167 (2004).

[122] A. Damascelli, Z. Hussain, and Z.-X. Shen, Rev. Mod. Phys. 75, 473 (2003).

[123] A. Mans, I. Santoso, Y. Huang, W. K. Siu, S. Tavaddod, V. Arpiainen, M. Lindroos, H. Berger, V. N. Strocov, M. Shi, L. Patthey, and M. S. Golden, Phys. Rev. Lett. 96, 107007 (2006). 
[124] A. Koitzsch, S. V. Borisenko, A. A. Kordyuk, T. K. Kim, M. Knupfer, J. Fink, M. S. Golden, W. Koops, H. Berger, B. Keimer, C. T. Lin, S. Ono, Y. Ando, and R. Follath, Phys. Rev. B 69, 220505(R) (2004).

[125] T. Kondo, T. Takeuchi, U. Mizutani, T. Yokoya, S. Tsuda, and S. Shin, Phys. Rev. B 72, $024533(2005)$.

[126] H. Ding, A. F. Bellman, J. C. Campuzano, M. Randeria, M. R. Norman, T. Yokoya, T. Takahashi, H. Katayama-Yoshida, T. Mochiku, K. Kadowaki, G. Jennings, and G. P. Brivio, Phys. Rev. Lett. 76, 1533 (1996).

[127] N. N. Kolesnikov, M. P. Kulakov, V. N. Molchanov, I. F. Schegolev, R. P. Shibaeva, V. I. Simonov, R. A. Tamazyan, and O. M. Vyasilev, Physica C 242, 385 (1995).

[128] N. N. Kolesnikov, V. E. Korotkov, M. P. Kulakov, R. P. Shibaeva, V. N. Molchanov, R. A. Tamazyan, and V. I. Simonov, Physica C 190, 219 (1992).

[129] H. Shaked, P. M. Keane, J.C. Rodriguez, F. F. Owen, R. L. Hitterman, and J. D. Jorgensen, Crystal Structures of the High-Tc Superconducting Copper-Oxides, Elsevier Science B.V., Amsterdam (1994).

[130] H. Krakauer and W. E. Pickett, Phys Rev. Lett. 60, 1665 (1988).

[131] O. K. Anderson, A. I, Liechtenstein, O. Jepsen, and F. Paulson, J. Phys. Chem. Solids 56, 1573 (1995).

[132] S. V. Borisenko, M. S. Golden, S. Legner, T. Pichler, C. Durr, M. Knupfer, J. Fink, G. Yang, S. Abell, and H. Berger, Phys. Rev. Lett. 84, 4453 (2000).

[133] M. R. Norman, M. Randeria, H. Ding, and J. C. Campuzano, Phys. Rev. B 52, 615 (1995).

[134] A. Kaminski, S. Rosenkranz, H. M. Fretwell, M. R. Norman, M. Randeria, J. C. Campuzano, J-M. Park, Z. Z. Li, and H. Raffy, Phys. Rev. B 73, 174511 (2006). 
[135] A. Bansil and M. Lindroos, Phys. Rev. Lett. 83, 5154 (1999).

[136] S. Kambe and O. Ishii, Physica C 341-348, 555 (2001).

[137] H. Eisaki, N. Kaneko, D. L. Feng, A. Damascelli, P. K. Mang, K. M. Shen, Z.-X. Shen, and M. Greven, Phys. Rev. B 69, 064512 (2004).

[138] S. Chakravarty, H. Kee, and K. Volker, Nature 428, 53 (2004).

[139] T. A. Zaleski and T. K. Kopec, Phys Rev. B 71, 014519 (2005).

[140] J. Mesot, M. R. Norman, H. Ding, M. Randeria, J. C. Campuzano, A. Paramekanti, H. M. Fretwell, A. Kaminski, T. Takeuchi, T. Yokoya, T. Sato, T. Takahashi, T. Mochiku, and K. Kadowaki, Phys. Rev. Lett 83, 840 (1999).

[141] H. Ding, J. C. Campuzano, A. F. Bellman, T. Yokoya, M. R. Norman, M. Randeria, T. Takahashi, H. Katayama-Yoshida, T. Mochiku, K. Kadowaki, and G. Jennings, Phys. Rev. Lett. 75, 1425 (1995).

[142] T. Kondo, T. Takeuchi, A. Kaminski, S. Tsuda, and S Shin, Phys. Rev. Lett. 98, 267004 (2007).

[143] H. Ding, M. R. Norman, T. Yokoya, T. Takeuchi, M. Randeria, J. C. Campuzano, T. Takahashi, T. Mochiku, and K. Kadowaki, Phys. Rev. Lett. 78, 2628 (1997).

[144] T. Hanaguri, C. Lupien, Y. Kohsaka, D.-H. Lee, M. Azuma, M. Takano, H. Takagi, and J. C. Davis, Nature 420, 1001 (2004).

[145] K. M. Shen, F. Ronning, D. H. Lu, F. Baumberger, N. J. C. Ingle, W. S. Lee, W. Meevasana, Y. Kohsaka, M. Azuma, M. Takano, H. Takagi, and Z.-X. Shen, Science 307, $901(2005)$.

[146] T. Hanaguri, Y. Kohsaka, J. C. Davis, C. Lupien, I. Yamada, M. Azuma, M. Takano, K. Ohishi, M. Ono, and H. Takagi, Nature Phys. 3, 865 (2007). 


\title{
CHAPTER 6. CONTROLLING THE CARRIER CONCENTRATION OF THE HIGH-TEMPERATURE SUPERCONDUCTOR $\mathrm{Bi}_{2} \mathrm{Sr}_{2} \mathrm{CaCu}_{2} \mathrm{O}_{8+\delta}$ IN ANGLE-RESOLVED PHOTOEMISSION SPECTROSCOPY EXPERIMENTS
}

A paper published in Physical Review B: Phys. Rev. B 81, 104521 (2010)

A. D. Palczewski ${ }^{1,2}$, Takeshi Kondo ${ }^{1,2}$, J. S. Wen ${ }^{3}$, G. Z. J. Xu ${ }^{3}$, G. Gu ${ }^{3}$, A. Kaminski ${ }^{1,2}$

\begin{abstract}
We study the variation of the electronic properties at the surface of a high-temperature superconductor as a function of vacuum conditions in angle-resolved photoemission spectroscopy experiments. Normally, under inadequate ultrahigh vacuum (UHV) conditions the carrier concentration of $\mathrm{Bi}_{2} \mathrm{Sr}_{2} \mathrm{CaCu}_{2} \mathrm{O}_{8+\delta}(\mathrm{Bi2212})$ increases with time due to the absorption of oxygen from $\mathrm{CO}_{2} / \mathrm{CO}$ molecules that are prime contaminants present in UHV systems. We find that in an optimal vacuum environment at low temperatures, the surface of Bi2212 is quite stable (the carrier concentration remains constant); however at elevated temperatures the carrier concentration decreases due to the loss of oxygen atoms from the Bi-O layer. These two effects can be used to control the carrier concentration in-situ. Our finding opens the possibility of studying the electronic properties of the cuprates as a function of doping across the phase diagram on the same surface of sample (i.e., with the same impurities and nondopant defects). We envision that this method could be utilized in other surface sensitive techniques such as scanning tunneling microscopy/spectroscopy.

\footnotetext{
${ }^{1}$ Division of Materials Science and Engineering, Ames Laboratory, Ames, Iowa 50011, USA

${ }^{2}$ Department of Physics and Astronomy, Iowa State University, Ames, Iowa 50011, USA

${ }^{3}$ Condensed Matter Physics and Materials Science Department, Brookhaven National Laboratory, Upton, New York 11973, USA
} 


\section{Introduction}

Surface techniques have played an important role in understanding the properties of the high temperature superconductors. They have revealed a number of fascinating phenomena such as the direct observation of the superconducting gap [147] and its anisotropy [148, 149], confirmation of the d-wave symmetry of the order parameter, direct observation of the pseudogap and its anisotropy [150-152], discovery of spatial inhomogeneities [153, 154], unusual spatial ordering [155] nodal quasiparticles [156], renormalization effects [157-159] and many others $[160,161]$. The successes of these techniques rely on the fact that the layers in some cuprates are very weakly bonded via the Van der Waals interaction. In such cases the bulk properties and surface properties are essentially identical, since there is no charge exchange between the layers. The samples in such cases can be thought of as a stack of very weakly electrically coupled 2-dimensional conducting surfaces rather than a 3-dimensional object. Two of the most commonly studied materials with this property are $\mathrm{Bi}_{2} \mathrm{Sr}_{2} \mathrm{CaCu}_{2} \mathrm{O}_{8+\delta}(\mathrm{Bi} 2212)$ and $\mathrm{Bi}_{2} \mathrm{Sr}_{2} \mathrm{CuO}_{6+\delta}$ (Bi2201). There is however one important aspect that needs to be carefully considered, namely the stability of the cleaved samples under ultra high vacuum (UHV) con-

ditions. UHV is a rather broad term and refers to pressures lower than $1 \times 10^{-9}$ Torr. Quite often such conditions are not sufficient to guarantee the stability of the surface, particularly in the case of non-stoichiometric materials such as the cuprates. These problems were recognized early on [148], and subsequent measurements revealed significant changes in the electronic properties as a function of time after cleaving. This issue was not carefully examined following these first measurements, and it is likely an important source of data discrepancies among the various groups [160, 161].

Here we present a systematic study of the electronic properties of Bi2212 as a function of vacuum conditions. There are significant difficulties with quantifying vacuum conditions in the deep UHV regime due to lack of common methods for absolute measurement of partial pressure. More significantly, the main problem with quantifying the vacuum conditions lies in fact that transport at very low pressures is ballistic in nature, thus the influx of molecules experienced by the sample surface and entrance of a residual gas analyzer (RGA) or ion gauge 
may be very different. The core of vacuum problems in an ARPES setup is the electron analyzer. It is typically the worst pumped part of the system, with many layers of shielding and electron surfaces. Gases desorbed inside of the analyzer exit via the electron lens and hit the cold surface of the sample. Those which don't stick are most likely pumped away before they get to the RGA/ion gauge. In the case of our setup this problem was solved by extensive baking of the analyzer (with a cumulative baking time of several months). We demonstrate that under less than ideal vacuum conditions (defined as a condition where the results of identical measurements very with time), increased carrier concentration arises due to the breakup of $\mathrm{CO}_{2} / \mathrm{CO}$ molecules by exposure to vacuum ultraviolet (VUV) photons and the subsequent adsorption of oxygen into the $\mathrm{BiO}$ layers. We demonstrate that this effect is most likely responsible for observation of bilayer splitting in Bi2212 in nominally "underdoped" samples. We show that when a tiny $\left(10^{-10}\right.$ Torr level) leak is introduced to the UHV system, the carrier concentration of the sample surface increases without exposure to VUV photons.

When the partial pressure of active gases is kept to optimal levels (again defined as the condition where the resulst of measurements do not change significantly with time), the lifetime of cleaved surface of Bi2212 can be as long as a few weeks at low temperatures $(T<150 \mathrm{~K})$. At elevated temperatures $(T>200 \mathrm{~K})$ the sample surface loses oxygen, which results in the reduction of carrier concentration. This second effect is most likely responsible for the recently reported non monotonic temperature dependence of the pseudogap [162], where at elevated temperatures the sample surface becomes underdoped and therefore develops a pseudogap. We demonstrate that these two effects (in-situ absorption and desorption of oxygen) can be utilized to control the carrier concentration of the sample surface. This approach enables one to study the intrinsic electronic properties of the cuprates across the phase diagram using a single cleave of the sample. This eliminates many extrinsic effects such as changing the chemical impurities and non-dopant defects. 


\section{Experimental Details}

The ARPES data were acquired using a laboratory-based Scienta 2002 electron analyzer and high intensity Gammadata UV4050 UV source with custom designed optics. The photocurrent at the sample was approximately $1 \mu \mathrm{A}$, which corresponds to roughly $10^{13}$ photons $/$ sec at $0.05 \%$ of the bandwidth. The energy resolution was set at $10 \mathrm{meV}$ and momentum resolution at $0.12^{\circ}$ and $0.5^{\circ}$ along a direction parallel and perpendicular to the analyzer slits, respectively. Samples were mounted on a variable temperature cryostat (10-300 K) cooled by a closed cycle refrigerator. The precision of the sample positioning stage was $1 \mu \mathrm{m}$. The partial pressure of the active gases was at the detection limit of the Residual Gas Analyzer (RGA) and the pressure of of a ion guage below $3 \times 10^{-11}$ Torr. Excellent vacuum conditions were achieved by strict adherence to good vacuum practices, use of UHV compatible materials and a cumulative bake-out time of the system in excess of 6 months. The typical lifetime of the optimally doped Bi2212 surfaces was greater than two weeks after cleaving, defined as less than $5 \%$ change of the superconducting gap $(2 \mathrm{meV})$ at $40 \mathrm{~K}$. The core-level spectra was acquired on the Hermon beam-line at the Synchrotron Radiation Center using a Scienta 2002 end-station. The photon energy was set at $500 \mathrm{eV}$ and energy resolution at $200 \mathrm{meV}$. The residual gas sperctrum was take by a SRS Quad Residual Gas Anylizer model RGA100 in CDEM (electron multiplier) mode, the factory stated pressure resolution is $1 \times 10^{-13}$ Torr which is constistant with the noise level in our data. 

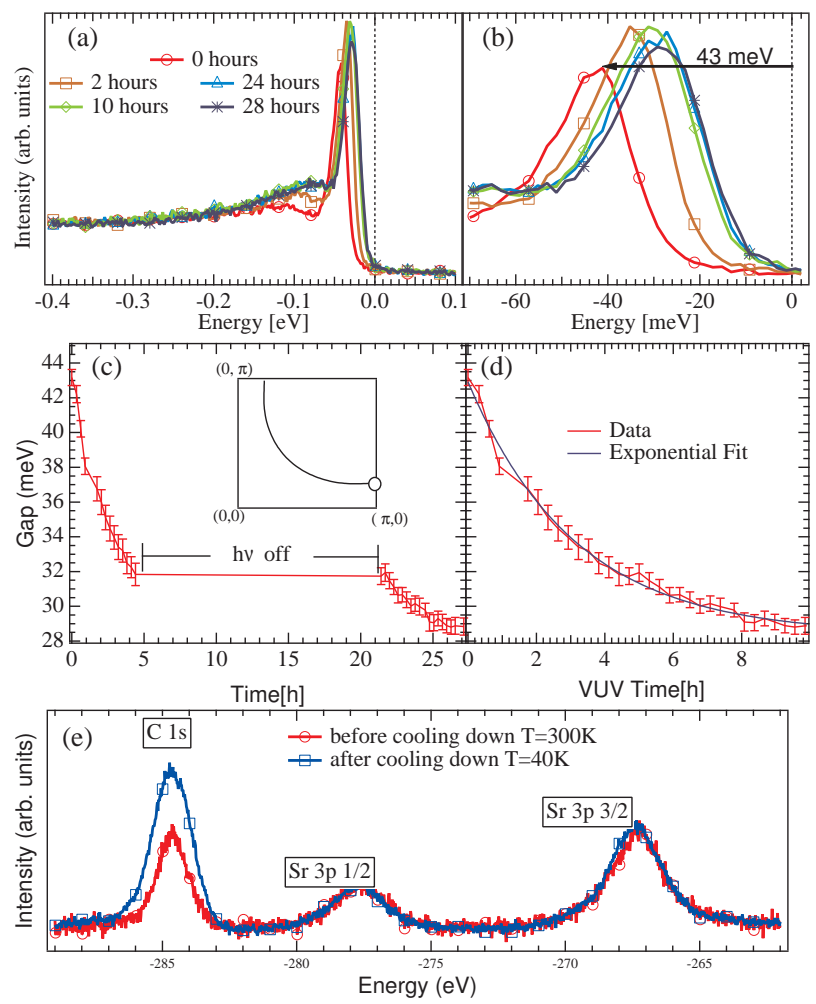

Figure 6.1 ARPES spectrum of Bi2212 taken under poor vacuum conditions $\left(\mathrm{CO}\right.$ and $\mathrm{CO}_{2}$ ): (a) sample EDC (energy distribution curves) taken at the antinode where the band crosses the Fermi energy at five different times (right after the cleave, 2, 10, 24, and 28 hours later ); (b) narrow view of (a); (c) time evolution of Bi2212's superconducting gap as a function of time; (d) the time evolution of Bi2212's superconducting gap under VUV photons (red) fitted with an exponential decay (blue), the temperature for (a)-(d) was set to $20 \mathrm{~K}$; and (e) C $1 \mathrm{~s}, \operatorname{Sr} 3 \mathrm{P}_{1} / 2$, $\mathrm{Sr} 3 \mathrm{p}_{3} / 2$ core level data from Bi2212 showing carbon deposits some time after cleaving and after cooling. 


\section{Increasing Carrier Concentration}

It has been known for some time that aging (increased surface doping) in cuprates is caused by less than ideal UHV conditions [148], that is, vacuum conditions where the spectrum is not stable as a function of time. Aging is usually detected by measuring the superconducting gap (the energy gap as defined by the difference between the peak position of a Bi2212 spectrum and the chemical potential measured by a polycrystalline gold sample) as a function of time. If the gap shifts to a lower binding energy the sample has aged [163, 164]. Figure 6.1 (a)(b) shows an example of this where a freshly cleaved Bi2212 single crystal was scanned in a less than optimal vacuum conditions to see how the spectrum changed over time. A shift to lower binding energy as well as a peak suppression (compared to comparable samples with the same gap size) was detected showing the sample was aging. In Figure 6.1 (c) the size of the superconducting gap is shown as a function of time. The aging occurred only when sample surface was illuminated with VUV photons. In the absence of VUV photons on the sample, (from 5th hour to 21st hour) the aging did not progress. If one considers only the time when the sample was exposed to VUV photons, the magnitude of the gap follows an exponential decay (blue line).

In absence of leaks, a UHV system has normally undetectable levels of oxygen as measured by a residual gas attached to the ARPES system (see Figure 6.2 (c) red spectrum). However in stainless steel vessels $\mathrm{CO}_{2} / \mathrm{CO}$ are always present. These oxide molecules can adhere to clean sample surfaces especially at low temperatures. When the molecules are exposed to VUV photons above $6 \mathrm{eV}$ they break into carbon and oxygen [165]; the oxygen can then be incorporated into $\mathrm{BiO}$ layer as dopant, while the carbon atoms remain on the surface. Support for this scenario is shown in Figure 6.1 (e) where the core-level spectrum of Bi2212 at $300 \mathrm{~K}$ and $40 \mathrm{~K}$ are shown. As the sample cooled more $\mathrm{CO}_{2} / \mathrm{CO}$ molecules adhered to the surface of the sample. Since there are carbon deposits some time after cleaving and even more after cooling, it is likely the oxygen accompanied the carbon to the surface. This oxygen can then change the doping of the sample after it is dissociated from the carbon.

In the presence of a leak a UHV system can have detectable amounts of oxygen. Under 

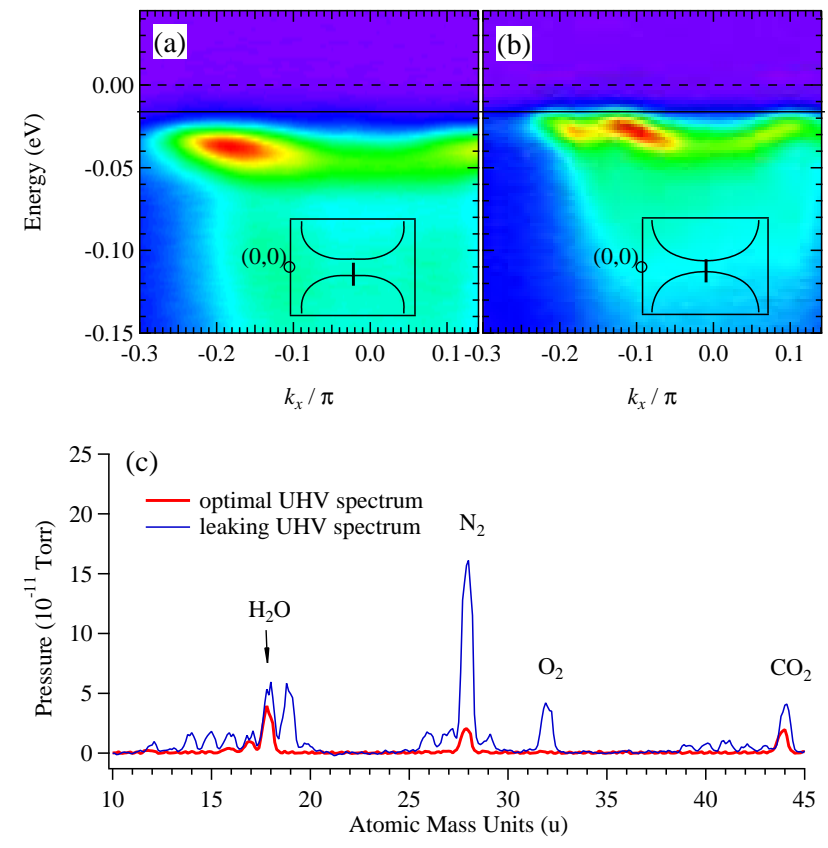

Figure 6.2 (a) ARPES intensity map of freshly cleaved optimally-doped Bi2212 at $(\pi, 0)$, showing no bilayer splitting; (b) ARPES intensity maps on the same sample and the same location as in (a) only oxygen aged (in-situ overdoping) in a UHV system with a leak, showing bilayer splitting and a peak shift location of the Fermi momentum (the black line serves as a guide to the eye); (c) residual gas analyzer (RGA) spectrum after an optimal bake-out (red curve) and after a bake-out where there was a $10^{-10}$ Torr air leak (blue curve).

these conditions a Bi2212 sample can age even without the breakdown of $\mathrm{CO}_{2} / \mathrm{CO}$. One of the trademarks of an over-doped (aged) Bi2212 sample is the appearance of bi-layer band splitting at the antinode $(\pi, 0)$. While there has been a relatively active discussion on whether Bi2212 contains bilayer band splitting all the time or just in an over-doped state; bilayer splitting has only been seen in over-doped samples when using a helium discharge lamp [166-169]. An example of this is shown in Figure 6.2 where a fresh Bi2212 sample was scanned and then allowed to sit in the leaky UHV system overnight before scanning again. Even though the sample was kept a $20 \mathrm{~K}$, bilayer band splitting was detected after the break, signaling that 
the sample aged because of oxygen absorption. An example Residual Gas Analyzer (RGA) spectrum contrasting the difference in the partial pressure of gases for an optimal UHV (red curve) and an UHV leak (blue) is shown in Figure 6.2 (c). The reader should notice that the leaky vacuum spectrum contains an $\mathrm{O}_{2}$ peak absent in the optimal vacuum spectrum, as well as excess $\mathrm{H}_{2} \mathrm{O}, \mathrm{CO}_{2}$ and $\mathrm{N}_{2} / \mathrm{CO}$. We know that the vacuum contains an small air leak $\left(10^{-10}\right.$ Torr) because of the ratio of $\mathrm{N}_{2}: \mathrm{O}_{2}$, which is $\approx 4: 1$, the same as in air.
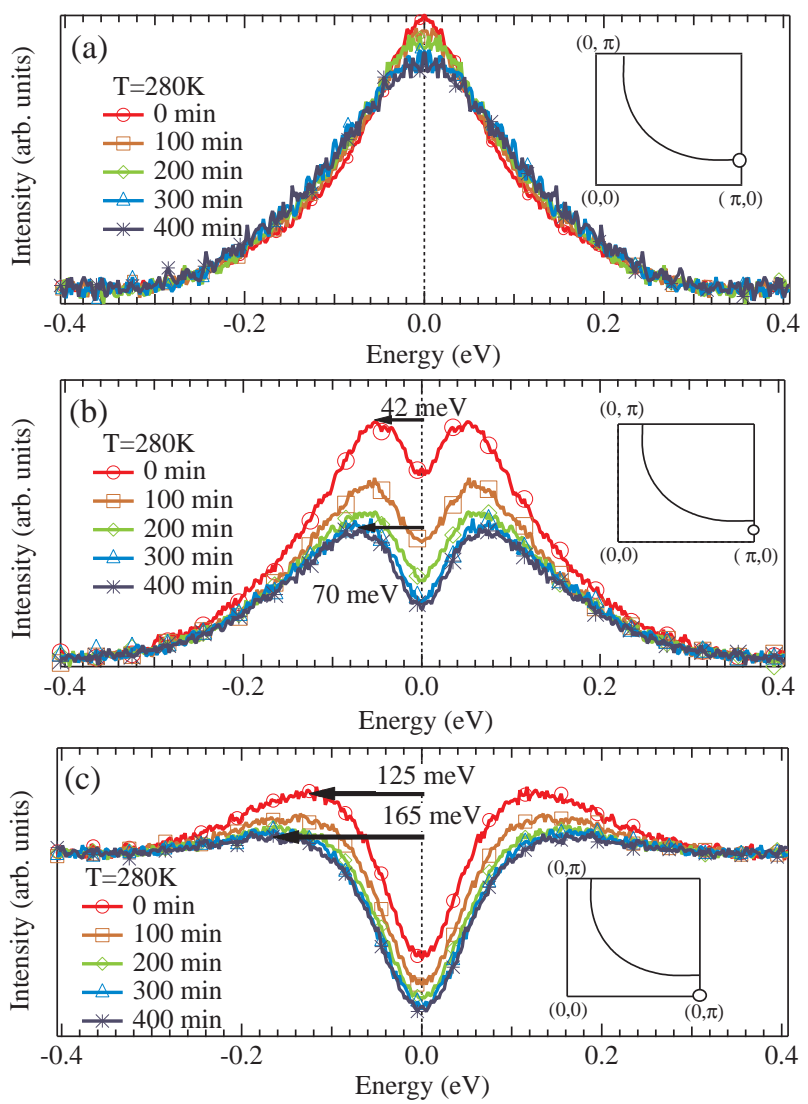

Figure 6.3 (a)-(c) symmetrized ARPES EDC's for Bi2212 taken at three points near $(\pi, 0)$, showing the time evolution of the spectrum at $280 \mathrm{~K}$. 


\section{Decreasing Carrier Concentration}

While in a reasonable vacuum system there can be enough $\mathrm{CO}_{2} / \mathrm{CO}$ to change the surface doping of a sample over time, in an ultra clean UHV system samples can live for many weeks without surface degradation or a change in doping (assuming the sample is kept at low temperature). Yet, when the sample is annealed above $200 \mathrm{~K}$ an interesting thing happens to the Bi2212's doping level; the sample doping level is reduced (the opposite of aging). This is seen in Figure 6.3 (a)-(c) where the time evolution of Bi2212's EDCs at three locations at or near $(\pi, 0)$ with the sample at $280 \mathrm{~K}$ is shown. The sample actually changes doping moving towards lower doping (signified by a larger spectral gap). Figure 6.4 (a) shows the energy distribution curve (EDC) at the antinodal Fermi momentum from the same sample before and after annealing at $280 \mathrm{~K}$ for 28 hours. The superconducting gap clearly shifts from $33 \mathrm{meV}$ to $41 \mathrm{meV}$ and the peak is suppressed, signaling that the doping has changed from a slightly over doped sample to a more under doped sample [170]. The momentum color maps from Figure 6.4 (a) are shown in Figure 6.4 (b)-(c); after annealing the gap shifts to higher binding energy, there is also a shift in the location of the Fermi momentum. This momentum shift comes from a change in the chemical potential, which moves lower in a rigid-band-like fashion upon doping [171].

Another way to see if a sample's carrier concentration has decreased is to look at the pseudogap. Figure 6.5 (a) shows the EDC at the Fermi momentum before and after annealing at $280 \mathrm{~K}$ for 28 hours. The pseudogap shifts from $30 \mathrm{meV}$ to $50 \mathrm{meV}$. As Bi2212 goes to lower doping levels the pseudogap becomes bigger and the temperature at which the pseudogap remains $\left(\mathrm{T}^{*}\right)$ becomes higher [149]. Figure 6.5 (b)-(c) demonstrates that before annealing $\mathrm{T}^{*}$ is below $140 \mathrm{~K}$ with the pseudogap disappearing and after annealing $\mathrm{T}^{*}$ is above $200 \mathrm{~K}$. The pseudogap transition temperature after annealing is above $200 \mathrm{~K}$, which guarantees that the sample is at a lower doping level.

Until now we have only shown the lowering of doping on Bi2212 at elevated temperature. While we still haven't shown if the doping change is caused by the elevated temperature or a combination of elevated temperature and VUV photons. This was tested by scanning the 

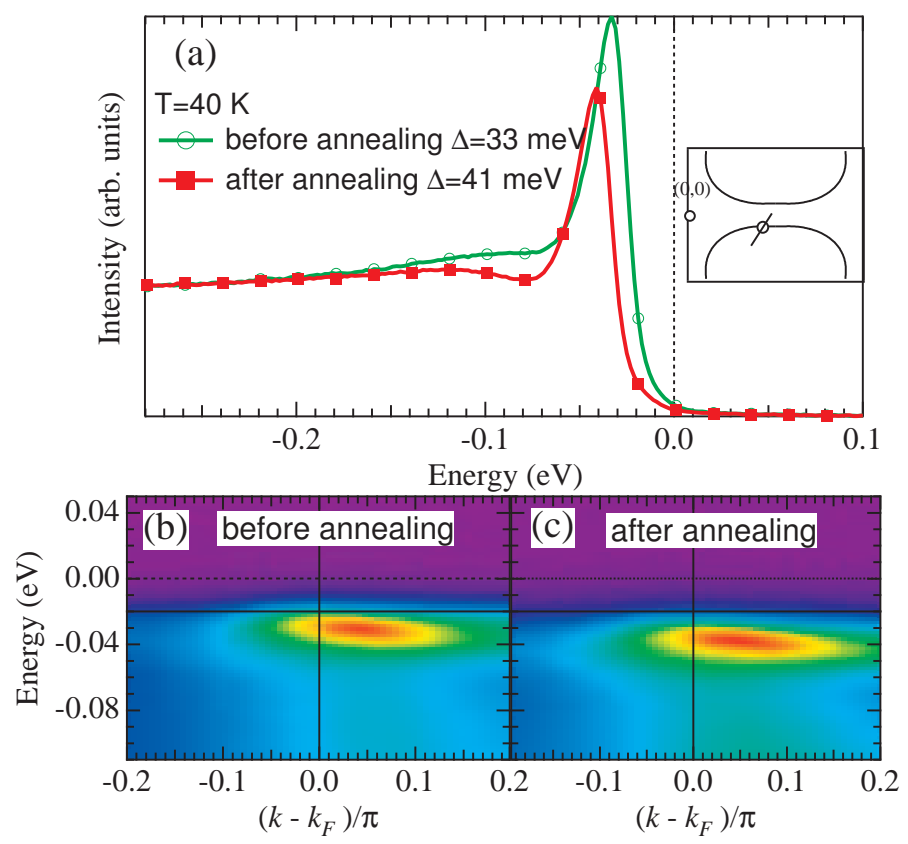

Figure 6.4 (a) EDC at the Fermi momentum close to the anti-node before (green circles) and after (solid red squares) annealing at $280 \mathrm{~K}$ over 28 hours with their respective superconducting gaps $\Delta$; (b)-(c) intensities maps taken about the Fermi momentum close to $(\pi, 0)$ before and after annealing.

sample just after cleaving and again after the sample sat under UHV for 16 days at $100 \mathrm{~K}$. This data is shown in Figure 6.6 (a). The spectrum barely changed over the two weeks. In Figure 6.6 (b) we show the $280 \mathrm{~K}$ spectrum just after cleaving, and again after the sample sat under UHV for 8 days at $280 \mathrm{~K}$. Most of the spectral weight has shifted to higher binding energies and the Fermi edge has all but disappeared, signifying an almost completely insulating sample. From Figure 6.6 we can conclude that the lowering of the sample's doping is only caused by the elevated temperatures.

The greatest consequence of this study is that Bi2212's doping can be change from overdoped all the way down to insulating in a systematic fashion on a single crystal. To this point the data presented has been on either a single sample overdoped by aging or underdoped by annealing. Figure 6.7 demonstrates how the same sample surface can be overdoped by aging 

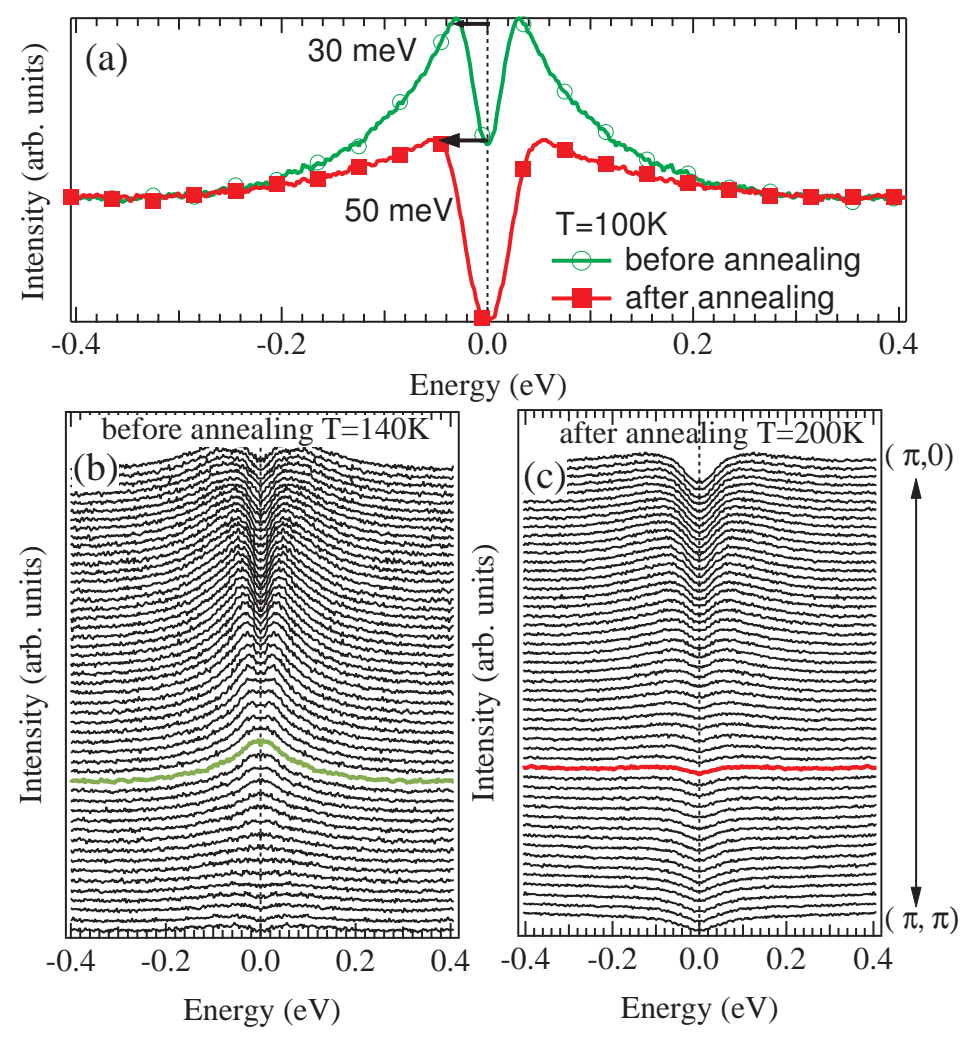

Figure 6.5 (a) $100 \mathrm{~K}$ symmetrized ARPES data taken at the Fermi momentum before and after annealing at $280 \mathrm{~K}$ for 28 hours; (b) ARPES intensities at $140 \mathrm{~K}$ before annealing; (c) ARPES intensities at $200 \mathrm{~K}$ after annealing.

and then under-doped by annealing to move across the phase diagram. An optimally doped Bi2212 sample was cleaved, the Fermi surface and superconducting gap values as a function of angle $\phi$ (angle clockwise from the line $(\pi,-\pi)$ to $(2 \pi,-\pi))$ was scanned [Figure 6.7 (a) \& (d)]. Aging was detected after a couple of days of scanning [Figure 6.7 (b) \& (e]). The sample was then annealed overnight at $280 \mathrm{~K}$ to remove the aging [Figure 6.7 (c) \& (f)].

\section{Conclusions}

We have presented a systematic study of the electronic properties at the surface of Bi2212 as a function of vacuum conditions. The results confirm that under poor vacuum conditions 

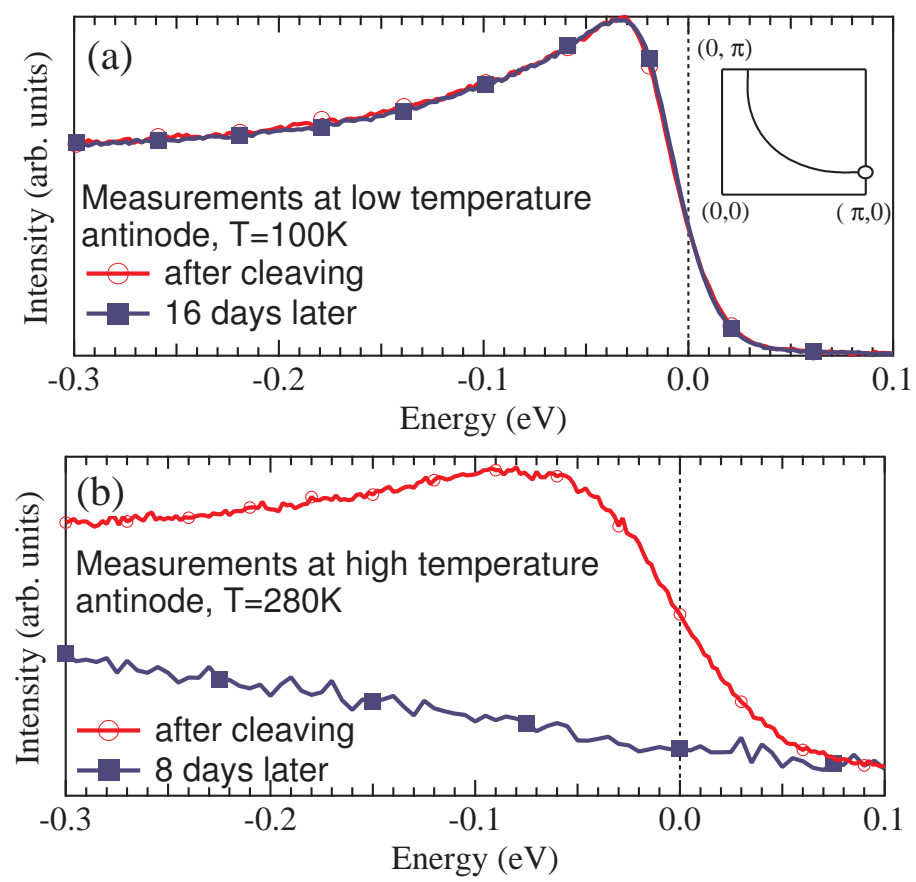

Figure 6.6 Bi2212 EDC at the Fermi momentum close to $(\pi, 0)$ (a) just after cleaving at $100 \mathrm{~K}$ (red circles) and again after sitting at $100 \mathrm{~K}$ for 16 days (solid blue squares), (b) just after cleaving at $280 \mathrm{~K}$ (red circles) and again after sitting at $280 \mathrm{~K}$ for 8 days (solid blue squares).

there is an increase in carrier concentration due to the breakup of $\mathrm{CO}_{2} / \mathrm{CO}$ molecules by exposure to vacuum ultra-violet (VUV) photons and a subsequent adsorption of oxygen into the $\mathrm{BiO}$ layers. We also show that with a UHV leak, where oxygen is present, a sample can increase its carrier concentration without exposure to VUV photons. This observation confirms that bilayer splitting only occurs in over-doped Bi2212. We then show that at elevated temperatures $(T>200 \mathrm{~K})$ the sample surface loses oxygen, which results in a reduction of the carrier concentration. These two effects (in-situ absorption and desorption of oxygen) can be utilized to control the carrier concentration of Bi2212. This approach enables one to study the intrinsic electronic properties (i.e. without changing the impurities and non-dopant defects) of the cuprates across the phase diagram in ARPES as well as other surface sensitive techniques 

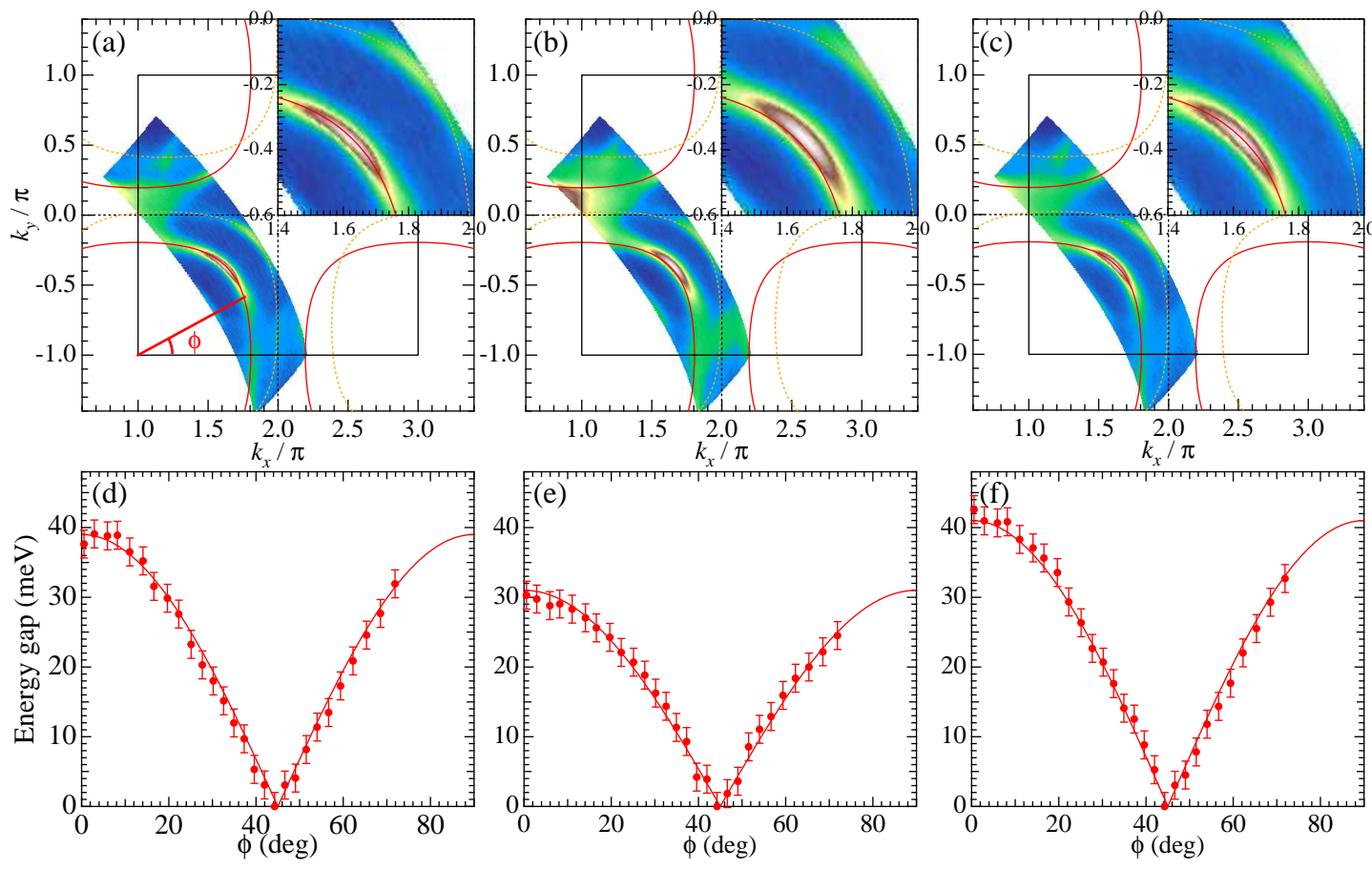

Figure 6.7 ARPES intensity plots at the Fermi energy from the same sample at three consecutive times all at $12 \mathrm{~K}$ : (a) just after cleaving, (b) after a couple of days of VUV aging at low temperature, (c) after annealing at $280 \mathrm{~K}$ overnight; the upper right hand corner of (a)-(c) are the zoomed in images from the bottom left hand corner, the red and dotted yellow curves are from a tight binding fit for optimally doped Bi2212 as a guide to the eye, (d)-(f) the size of the superconducting gap as a function of angle $\phi$ from (a)-(c) respectively.

using a single cleaved surface.

\section{Acknowledgments}

This work was supported by Director Office for Basic Energy Sciences, US DOE. Work at Ames Laboratory was supported by the Department of Energy - Basic Energy Sciences under Contract No. DE-AC02-07CH11358. The work at BNL was supported by Department of Energy - Basic Energy Sciences under Contract No. DE-AC02-98CH10886. Synchrotron Radiation Center is supported by the National Science Foundation under award No. DMR- 
0537588 .

\section{Bibliography}

[147] C. G. Olson, R. Liu, A. -B. Yang, D. W. Lynch, A. J. Arko, R. S. List, B. W. Veal, Y. C. Chang, P. Z. Jiang, and A. P. Paulikas, Science 245, 731 (1989).

[148] Z.-X. Shen, D. S. Dessau, B. O. Wells, D. M. King, W. E. Spicer, A. J. Arko, D. S. Marshall, L. W. Lombardo, A. Kapitulnik, P. Dickinson, S. Doniach, J. Dicarlo, T. Loeser, and C. H. Park, Phys. Rev. Lett. 70, 1553 (1993).

[149] H. Ding, M. R. Norman, J. C. Campuzano, M. Randeria, A. F. Bellman, T. Yokoya, T. Takahashi, T. Mochiku, and K. Kadowaki, Phys, Rev. B 54, R9678 (1996).

[150] H. Ding, T. Yokoya, J. C. Campuzano, T. Takahashi, M. Randeria, M. R. Norman, T. Mochiku, K. Kadowaki, and J. Giapintzakis, Nature (London) 382, 51 (1996).

[151] A. G. Loeser, Z.-X. Shen, D. S. Dessau, D. S. Marshall, C. H. Park, P. Fournier, and A. Kapitulnik, Science 273, 325-329 (1996).

[152] M. R. Norman, H. Ding, M. Randeria, J. C. Campuzano, T. Yokoya, T. Takeuchi, T. Takahashi, T. Mochiku, K. Kadowaki, P. Guptasarma, and D. G. Hinks, Nature (London) 392, 157 (1998).

[153] S. H. Pan, J. P. O’Neal, R. L. Badzey, C. Chamon, H. Ding, J. R. Engelbrecht, Z. Wang, H. Eisaki, S. Uchida, A. K. Gupt,a K.-W. Ng, E. W. Hudson, K. M. Lang, and J. C. Davis, Nature 413, 282 (2001).

[154] K. K. Gomes, A. N. Pasupathy, A. Pushp, S. Ono, Y. Ando, and A. Yazdani, Nature (London) 447, 569 (2007).

[155] T. Hanaguri, C. Lupien, Y. Kohsaka, D. -H. Lee, M. Azuma, M. Takano, H. Takagi, and J. C. Davis, Nature 430, 1001 (2004). 
[156] A. Kaminski, J. Mesot, H. Fretwell, J. C. Campuzano, M. R. Norman, M. Randeria, H. Ding, T. Sato, T. Takahashi, T. Mochiku, K. Kadowaki, and H. Hoechst, Phys. Rev. Lett. 84, $1788(2000)$.

[157] T. Valla, T. E. Kidd, W.-G. Yin, G. D. Gu, P. D. Johnson, Z. -H. Pan, and A. V. Fedorov, Phys. Rev. Lett. 98, 167003 (2007).

[158] P. V. Bogdanov, A. Lanzara, S. A. Kellar, X. J. Zhou, E. D. Lu, W. J. Zheng, G. Gu, J.-I. Shimoyama, K. Kishio, H. Ikeda, R. Yoshizaki, Z. Hussain, and Z.-X. Shen, Phys. Rev. Lett. 85, 2581 (2000).

[159] A. Kaminski, M. Randeria, J. C. Campuzano, M. R. Norman, H. Fretwell, J. Mesot, T. Sato, T. Takahashi, and K. Kadowaki, Phys. Rev. Lett. 86, 1070 (2001).

[160] A. Damascelli, Z. Hussain, and Z.-X. Shen, Rev. Mod. Phys. 75, 473 (2003).

[161] J. C. Campuzano, M. R. Norman, and M. Randeria, in The Physics of Superconductors, Vol. 2, ed. K. H. Bennemann and J. B. Ketterson (Springer, Berlin), p. 167 (2004).

[162] A. A. Kordyuk, S. V. Borisenko, V. B. Zabolotnyy, R. Schuster, D. S. Inosov, R. Follath, A. Varykhalov, L. Patthey, and H. Berger, Phys. Rev. B 79, 020504(R) (2009).

[163] H. Ding, M. R. Norman, T. Yokoya, T. Takeuchi, M. Randeria, J. C. Campuzano, T. Takahashi, T. Mochiku, and K. Kadowaki, Phys. Rev. Lett. 78, 2628 (1997).

[164] P. Schwaller, T. Greber, P. Aebi, J. M. Singer, H. Berger, L. Forr, and J. Osterwalder, Eur Phys. J. B 18, 215 (2000).

[165] M. M. Halmann, and M. Steinberg, Greenhouse Gas Carbon Dioxide Migration (CRC, Boca Raton, Florida, p. 47 (1998).

[166] Y.-D. Chuang, A. D. Gromko, A. V. Fedorov, Y. Aiura, K. Oka, Yoichi Ando, M. Lindroos, R. S. Markiewicz, A. Bansil, and D. S. Dessau, Phys. Rev. B 69, 094515 (2004). 
[167] S. V. Borisenko, A. A. Kordyuk, S. Legner, T. K. Kim, M. Knupfer, C. M. Schneider, J. Fink, M. S. Golden, M. Sing, R. Claessen, A. Yaresko, H. Berger, C. Grazioli, and S. Turchini, Phys. Rev. B 69, 224509 (2004).

[168] S. V. Borisenko, A. A. Kordyuk, A. Koitzsch, J. Fink, J. Geck, V. Zabolotnyy, M. Knupfer, B. Büchner, H. Berger, M. Falub, M. Shi, J. Krempasky, and L. Patthey, Phys. Rev. Lett. 96, 067001 (2006).

[169] A. A. Kordyuk, S. V. Borisenko, A. N. Yaresko, S.-L. Drechsler, H. Rosner, T. K. Kim, A. Koitzsch, K. A. Nenkov, M. Knupfer, J. Fink, R. Follath, H. Berger, B. Keimer, S. Ono, and Y. Ando, Phys. Rev. B 70, 214525 (2004).

[170] T. Sato, T. Kamiyama, Y. Naitoh, T. Takahashi, I. Chong, T. Terashima, and M. Takano, Phys. Rev. B 63, 132502 (2001).

[171] M. Hashimoto, T. Yoshida, H. Yagi, M. Takizawa, A. Fujimori, M. Kubota, K. Ono, K. Tanaka, D. H. Lu, Z.-X. Shen, S. Ono, and Yoichi Ando, Phys. Rev. B 77, 094516 (2008). 


\title{
CHAPTER 7. PROBING THE PARTICLE-HOLE SYMMETRY OF THE PSEUDOGAP IN HIGH TEMPERATURE SUPERCONDUCTING CUPRATES
}

A paper in preparation for Physical Review Letters

A. D. Palczewski ${ }^{1,2}$, Takeshi Kondo ${ }^{1,2}$, J. S. Wen ${ }^{3}$, G. Z. J. Xu ${ }^{3}$, G. Gu ${ }^{3}$, A. Kaminski ${ }^{1,2}$

\begin{abstract}
We used angle resolved photoemission spectroscopy (ARPES) to investigate the particlehole symmetry of cuprate superconductors in the pseudogap state. Our data show a particlehole symmetric pseudogap close to $(\pi, 0)$ which symmetrically closes before the node $(\pi, \pi)$, creating disconnected arcs rather than closed pockets on the Fermi surface (FS). In the underdoped samples, the gap is closed where the FS deviates from the nested region close to $(\pi, 0)$. The gap data are consistent with a charge density wave (CDW) origin of the pseudogap, where a gap opens because of nesting.
\end{abstract}

\section{Introduction}

In conventional superconductors, a symmetric particle-hole energy gap forms at the chemical potential below the superconducting transition temperature $\left(T_{c}\right)$. This gap corresponds to the energy needed to break a Cooper pair into two excited electrons [172]. In high temperature superconducting cuprates, below $T_{c}$, this gap is characterized by a large quasiparticle

\footnotetext{
${ }^{1}$ Division of Materials Science and Engineering, Ames Laboratory, Ames, Iowa 50011, USA

${ }^{2}$ Department of Physics and Astronomy, Iowa State University, Ames, Iowa 50011, USA

${ }^{3}$ Condensed Matter Physics and Materials Science Department, Brookhaven National Laboratory, Upton, New York 11973, USA
} 
peak in the energy spectrum close to $E_{F}$ and a d-wave order parameter (creating 4 points along the $(\pi, \pi)$ direction of the Fermi surface). In optimally and underdoped samples at temperatures above $T_{c}$ another gap is observed - the pseudogap [173]. This gap is characterized by broad spectral features without a quasi-particle peak and a gap shape that changes with doping. The particle-hole symmetry of the pseudogap is controversial, with multiple theories being proposed. Some suggest the pseudogap should have the same particle-hole symmetry as the superconducting gap because the pseudogap is a preformed superconducting pair state [174-176]. Others suggest there should be particle-hole symmetry breaking as the pseudogap represents a competing state [177-180].

Previous studies probing the symmetry of the pseudogap also produced conflicting results; some see particle-hole symmetry [173, 181-186], while others show (or possibly show) particlehole symmetry breaking $[172,182,183,187]$. In the symmetry breaking scenario the FS might be comprised of four closed pockets in the $(\pi, \pi)$ direction of the Brillouin zone; similar to pockets seen in quantum oscillations measurements [178]. In the particle-hole symmetric scenario the FS is made up of four disconnected Fermi arcs in the $(\pi, \pi)$ direction of the Brillouin zone; similar to gapped arcs interpreted from other quantum oscillations measurements [188]. To add to the confusion, recent angle-resolved photoemission spectroscopy (ARPES) experiments produce disconnected arcs, and unrelated Fermi pockets on the same FS [189].

In this letter we used ARPES to investigate the particle-hole symmetry of cuprates in the pseudogap state by looking at the thermally excited states above the Fermi level $\left(E_{F}\right)$. Our data show no sign of particle-hole symmetry breaking at any location on the FS. The particlehole symmetric data lead to a FS made up of disconnected arcs rather than closed pockets. The gap structure is constant with a charge density wave (CDW) origin of the pseudogap where the nested region close to the antinode forms the gap.

\section{Experiment}

The $\mathrm{Bi}_{2} \mathrm{Sr}_{2} \mathrm{CaCu}_{2} \mathrm{O}_{8+\delta}(\mathrm{Bi} 2212)$ and $(\mathrm{Bi}, \mathrm{Pb})_{2}(\mathrm{Sr}, \mathrm{La})_{2} \mathrm{CuO}_{6+\delta}(\mathrm{Bi} 2201)$ single crystals used in this study were grown by the conventional floating-zone (FZ) method. All ARPES data 
were acquired using the laboratory-based Scienta 2002 electron analyzer and high intensity Gammadata UV4050 UV source with custom-designed optics at Iowa State University/Ames Laboratory, Ames, IA, USA. All data was acquired using the HeI line with photon energy of 21.2 $\mathrm{eV}$ and with the analyzer set to ADC mode. The energy resolution was $\sim 6 \mathrm{meV}$ and momentum resolution at $0.12^{\circ}$ and $0.5^{\circ}$ along a direction parallel and perpendicular to the analyzer slits, respectively. The samples were kept in an UHV environment where partial pressure of active gases remained below $10^{-11}$ Torr [190]. The high temperature ARPES data are divided by a temperature dependent Fermi function convoluted with a Gaussian representing the finite energy resolution of the analyzer. By dividing the data by a temperature-dependent Fermi function, the states above $\sim 4 \times \mathrm{k}_{B} T(45 \mathrm{meV} @ 140 \mathrm{~K})$ of $E_{F}$ are revealed [183]. The Fermi level for division was extracted from a polycrystalline sample of gold at each acceptance angle. The data was divided after the experimental background inherent in our analyzer and light source was subtracted and normalized.

\section{Data}

Figure 7.1 shows the full 3-D electronic structure $\left(k_{x}, k_{y}, E\right)$ of Bi2212 UD75K and Bi2201 UD20K above and below the Fermi level taken in the second zone at $140 \mathrm{~K}$. The experimental cutting location on the Bi2212 UD75 K sample is shown in Figure 7.1 (a). Each circle and line represent the location of $k_{F}$ and the experimental cutting direction respectively. The open circles are locations of a gapped FS and the closed circles are the non-gapped FS where the dip at $E_{F}$ disappears when dividing by the Fermi Function. The diagonal line from $(1,0)$ to $(2,-1)$ represent the antiferromagnetic zone boundary found in the parent compound and the lines from $(1,-1)$ to $(2,0)$ is the symmetry line about the node. From the 2-D image plots in Figure 7.1 (b), the exact band shape and gap symmetry is revealed (to enhance the contrast ratio each panel was independently normalized). The bands close to the antinode $(\pi, 0)$ have a large particle-hole symmetric gap which smoothly closes before the node $(\pi, \pi)$, creating an arc on the FS. In all cuprates the pseudogap is thought to come from the same origins, and for this reason we took a nearly identical data set from Figure 7.1 (a)-(b) on single layer Bi2201 

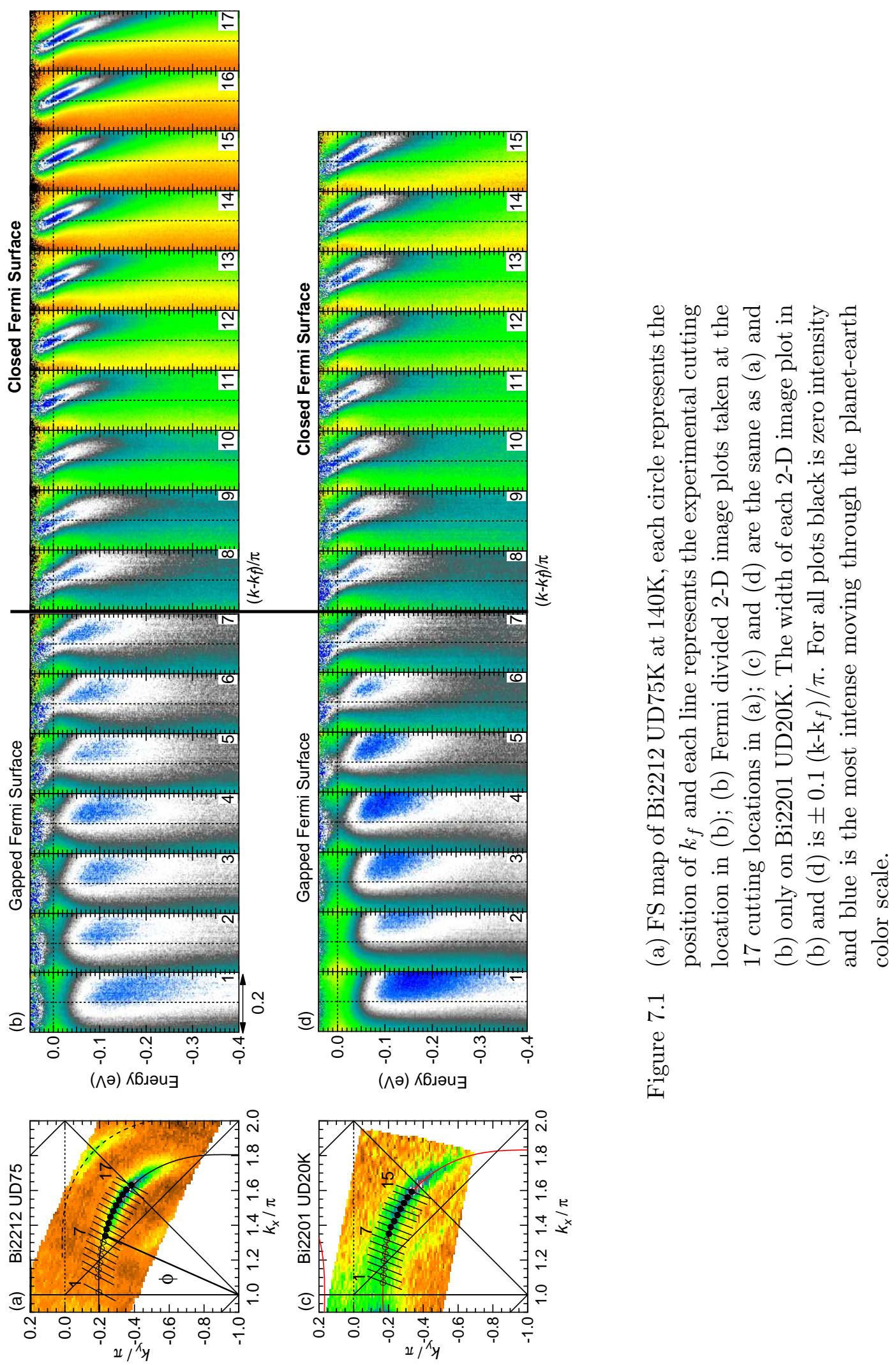
[Figure 7.1 (c)-(d)]. The band structure is nearly identical between the two samples. The gap closes where the nested region (parallel part) of the FS ends and the main band bends towards the node. In addition, the antiferromagnetic zone boundary does not affect the particle-hole symmetry in any way.

When looking at 2-D image plots the exact particle-hole symmetry can be difficult to interpret. For this reason the energy distribution curves (EDC) at $k_{F}$ are used to ensure the proper symmetry. Figure 7.2 shows the EDC's from the $17 k_{F}$ locations in Figure 7.1 (a)-(b) and $15 k_{F}$ locations in Figure $7.1(\mathrm{c})-(\mathrm{d})$. The location of $k_{F}$ was found in two ways; either where the smallest gap forms when looking at the EDCs or by fitting the peak position in the momentum distribution curves (MDC) at $E_{F}$ after Fermi division. Close to the antinode (cut 1-8) the gap can be quite large, and finding the exact location of $k_{f}$ is difficult with the MDC method. This is why the smallest gap in the EDCs is chosen to find $k_{f}$. Closer to the node (cuts 10-17 or 10-15 in Figure 7.2) $k_{f}$ is easier to find because the gap is closed, and $k_{f}$ is easily extracted from the MDC at $E_{F}$. Finding $k_{f}$ in the crossover region at the end of the Fermi arc (cuts 6-9 in Figure 7.2) might be difficult from MDC fits if the gap shifts. Since the data shows a symmetric particle-hole gap such ambiguity in not relevant and the location of the smallest gap or MDC peak can be used. We note, when $k_{f}$ is off by a couple of channels the bottom of the gap is always at $E_{F}$, this is in contrast to a symmetry breaking gap where the bottom of the gap would be located away from $E_{F}$ and appear to shift. This is important because of a recent ARPES study suggesting that $k_{f}$ changes at the antinode which could in principle cause symmetry breaking [172]. After the gap closes (no dip at $E_{F}$ ) the EDC's become more intense at $E_{F}$ moving toward the node $(\pi, \pi)$ (cuts 17 or 15$)$. In the intermediate cuts after the gap closes (e.g. 8-11), the EDC shape does not match the overall shape closer to the node. This suggests that the true peak-to-peak gap is still present despite the lack of a clear dip in the EDCs [174].

Multiple experimental papers suggest there should be particle-hole symmetry breaking, specifically close to the end of the Fermi arc in the first zone $[182,183,187]$. Because of the inconsistency between our symmetric second zone data and the particle-hole symmetry 

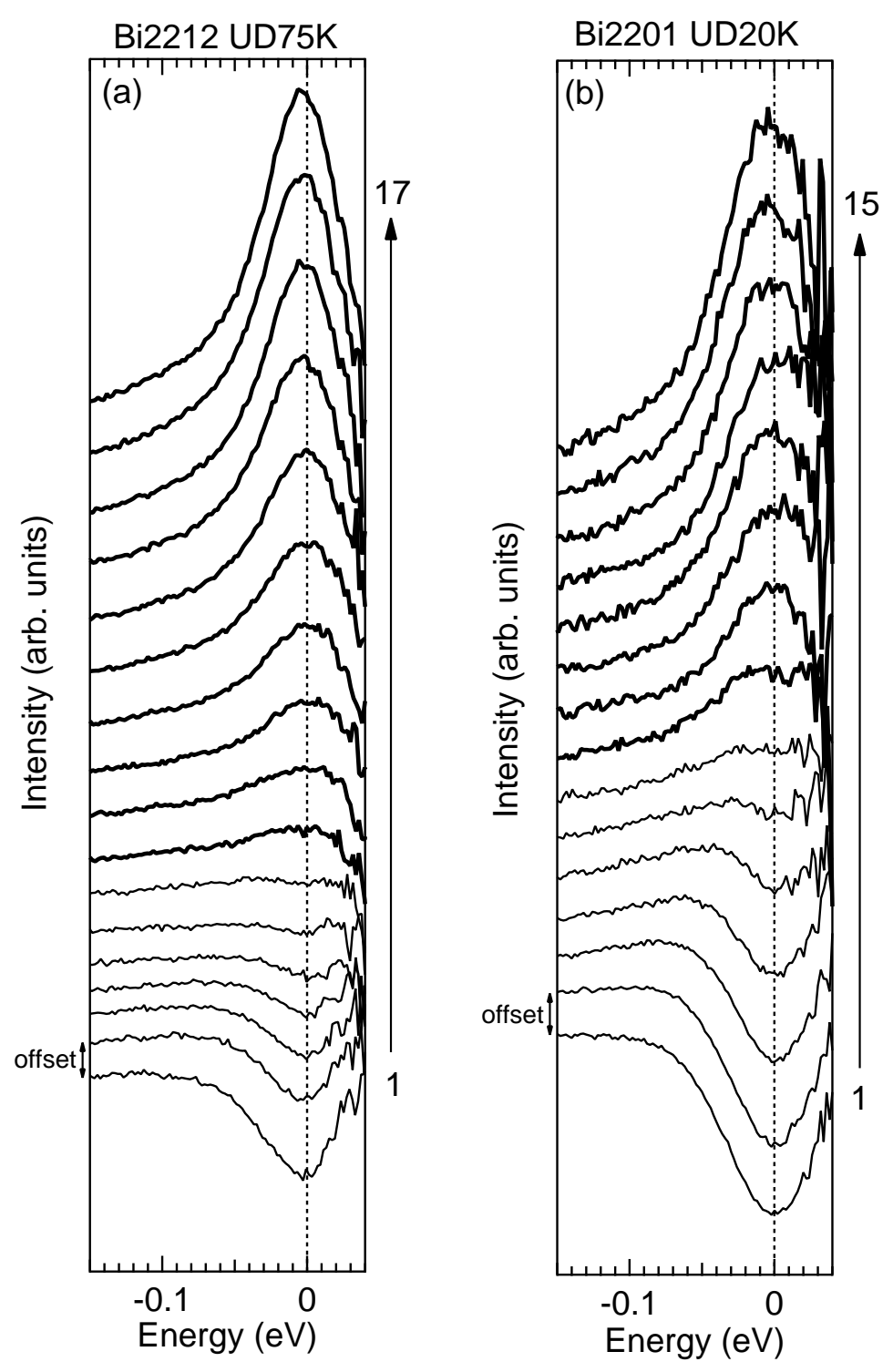

Figure 7.2 Fermi divided energy distribution curves (EDC) from Figure 7.1. (a) Bi2212 UD75K taken at $140 \mathrm{~K}$ at the $17 k_{f}$ pointing in Figure 7.1 (a). (b) Bi2201 UD20K taken at $140 \mathrm{~K}$ at the 15 $k_{f}$ points in Figure 7.1 (c). The thin curves, $1-7$ in (a) and (b) are locations of a gapped band, and the bold curves, 8-17 in (a) and 8-15 in (b) are locations of a closed gap. 


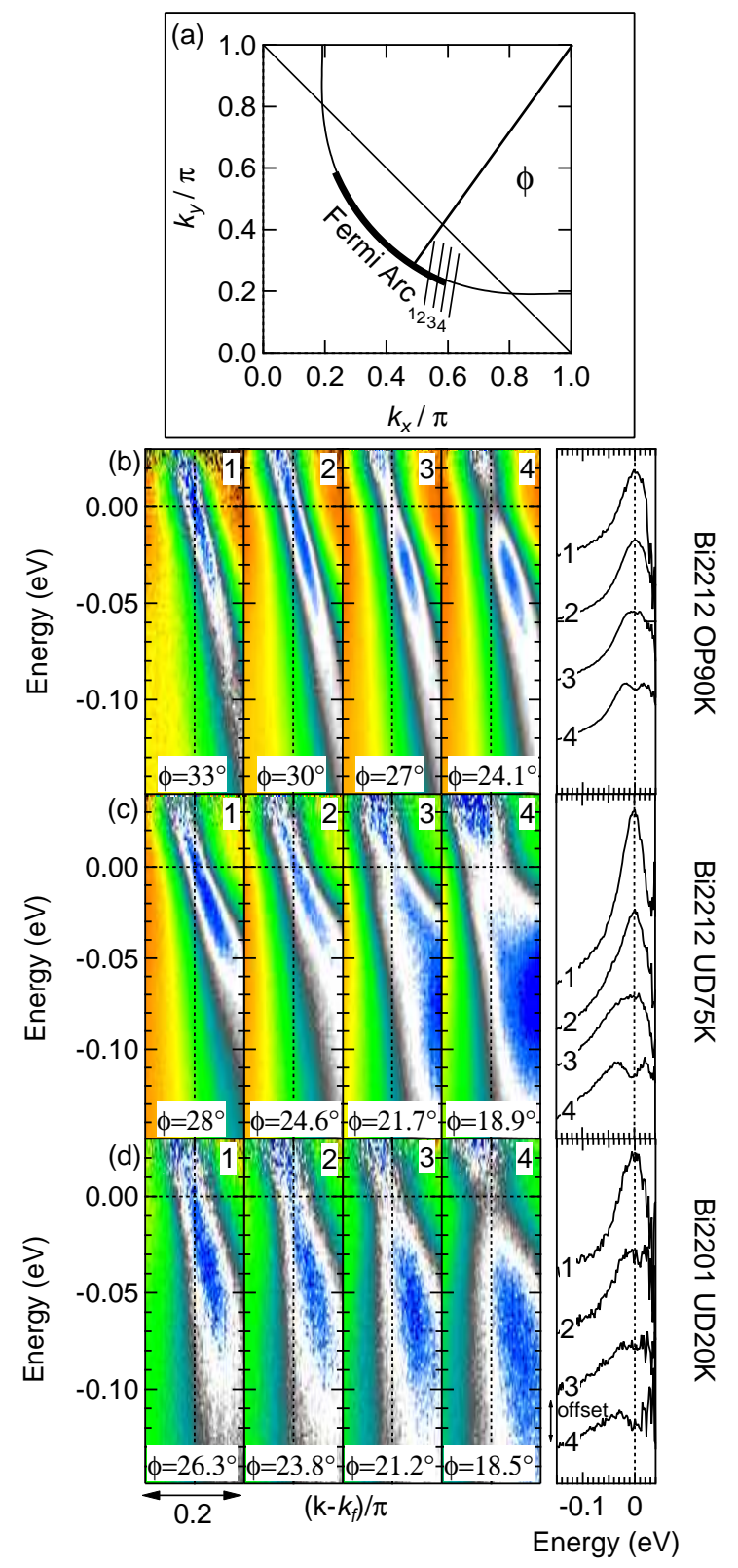

Figure 7.3 (a) Schematic location of the 4 cuts close to the end of the Fermi arc for (b)-(c). (b) Bi2212 OP90K taken at $110 \mathrm{~K}$ from the cutting locations in (a) taken in zone 1. (c) the same as (b) only on Bi2212 UD75K at $140 \mathrm{~K}$. (d) the same as (c) only on Bi2201 UD20K in the second zone [reflected data (identical) from Figure 7.1 (d) data (6-9)]. The width of each 2-D image plot in (b) and (d) is $\pm 0.1\left(k-k_{f}\right) / \pi$, with the vertical line at $k_{f}$. For all plots black is zero intensity and blue is the most intense moving through the planet earth color scale. 
breaking from other studies we present Figure 7.3. Figure 7.3 probes two more Bi2212 samples (OP90K and UD75K) in the first zone at the end of the Fermi arc. In addition, the Bi2201 second zone data [Figure 7.1 (d)] close to the end of the arc are also reproduced in Figure 7.3 (d). In each sample the exact cutting angle at $k_{f}$ is shown at the bottom of each image plot. Moving from left to right (1-4) each sample shows a closed gap which symmetrically opens around point 3. Again the data shows particle-hole symmetry no matter the scanning zone or cutting direction.

Finally, we would like to comment on the possible theoretical origins of the pseudogap given our particle-hole symmetric data. The most obvious explanation would be a preformed superconducting pair state in which a $d$-wave superconducting gap at elevated temperature and with lifetime broadening would appear similar to the data [174]. Yet, there is mounting evidence that the pseudogap represents a competing states which makes the preformed pair explanation unlikely [173, 191-193]. Our data contain some features which make a charge density wave (CWD) origin of the pseudogap likely. From [Figures 7.1 (a) \& (c)] the gap only closes after the FS deviates from the nested region close to the antinode. This observation is similar to STM measurements which show a CDW checkerboard pattern in the pseudogap state. The checkerboard pattern appears with a real space periodicity which corresponds to the reciprocal space nested FS location close the antinode [194]. Some might argue the data is inconsistant with a CDW scenario because particle-hole symmetry breaking is also possible within the model $[172,177,179]$. There is another possibility which would include a yet unknown interaction that creates a particle-hole symmetric pseudogap. There is a need for further detailed studies of the spectral properties beyond particle-hole symmetry to determine such interactions.

\section{Conclusions}

In conclusion, this letter reported on the particle-hole symmetry of the pseudogap in cuprate superconductors. We found no signature of particle-hole symmetry breaking at any location on the FS. The data reaffirm the fact that the pseudogap's FS is comprised of disconnected arcs 
rather than closed pockets. Our data point to a pseudogap which arises from CDW scattering close at the nested FS close, consistent with STM checkerboard patterns seen in the pseudogap state.

\section{Acknowledgments}

We thank R. M. Fernandes and J. Schmalian for their useful discussions. This work was supported by the Director's Office for Basic Energy Sciences, US DOE. Work at Ames Labo-

ratory was supported by the Department of Energy - Basic Energy Sciences under Contract No. DE-AC02-07CH11358. The work at BNL was supported by Department of Energy - Basic Energy Sciences under Contract No. DE-AC02-98CH10886.

\section{Bibliography}

[172] Makoto Hashimoto, Rui-Hua He, Kiyohisa Tanaka, Jean-Pierre Testaud, Worawat Meevasana, Rob G. Moore, Donghui Lu, Hong Yao, Yoshiyuki Yoshida, Hiroshi Eisaki, Thomas P. Devereaux, Zahid Hussain and Zhi-Xun Shen, Nature Physics 6, 414 (2010).

[173] W. S. Lee, I. M. Vishik, K. Tanaka, D. H. Lu, T. Sasagawa, N. Nagaosa, T. P. Devereaux, Z. Hussain, and Z.-X. Shen, Nature 450, 81 (2007).

[174] M. R. Norman, A. Kanigel, M. Randeria, U. Chatterjee, and J. C. Campuzano, Phys. Rev B 76, 174501 (2007).

[175] H. Alloul, A. Mahajan, H. Casalta, and O. Klein, Phys. Rev. Lett. 70, 1171 (1993).

[176] M. R. Norman, D. Pines, and C. Kallin, Adv. Phys. 54, 715 (2005).

[177] Sudip Chakravarty, Chetan Nayak, and Sumanta Tewari, Phys. Rev. B 68, 100504(R) (2003).

[178] David LeBoeuf, Nicolas Doiron-Leyraud, Julien Levallois, R. Daou, J.-B. Bonnemaison, N. E. Hussey, L. Balicas, B. J. Ramshaw, Ruixing Liang, D. A. Bonn, W. N. Hardy, S. Adachi, Cyril Proust, and Louis Taillefer, Nature 450, 533 (2007). 
[179] S. Chakravary, R. B. Laughlin, D. K. Morr, and C. Nayak, Phys. Rev. B 63, 094503 (2001).

[180] Jian-Xin Li, Chang-Qin Wu, and Dung-Hai Lee, Phys. Rev. B 74, 184515 (2006).

[181] Kiyohisa Tanaka, W. S. Lee, D. H. Lu, A. Fujimori, T. Fujii, Risdiana, I. Terasaki, D. J. Scalapino, T. P. Devereaux, Z. Hussain, and Z.-X. Shen, Science 314, 1910 (2006).

[182] H.-B. Yang, J. D. Ramaeu, Z.-H. Pan, G. D. Gu, P. D. Johnson, R. H. Claus, D. G. Hinks, and T. E. Kidd, arXiv:1008.3121v2 (2010).

[183] H.-B. Yang, J. D. Rameau, P. D. Johnson, T. Valla, A. Tsvelik, and G. D. Gu, Nature 456, 77 (2008).

[184] A. Kanigel, U. Chatterjee, M. Randeria, M. R. Norman, G. Koren, K. Kadowaki, and J. C. Campuzano, Phys. Rev. Lett. 101, 137002 (2008).

[185] T. Sato, T. Kamiyama, T. Takahashi, J. Mesot, A. Kaminski, J. C. Campuzano, H. M. Fretwell, T. Takeuchi, H. Ding, I. Chong, T. Terashima, and M. Takano, Phys. Rev. B 64, $054502(2001)$.

[186] T. Takahashi, T. Sato, H Matsui, and K. Terashima, New J. Phys. 7, 105 (2005).

[187] M. Shi, A. Bendounan, E. Razzoli, S. Rosenkranz, M. R. Norman, J. C. Campuzano, J. Chang, M. Mansson, Y. Sassa, T. Claesson, O. Tjernberg, L. Patthey, N. Momono, M. Oda, M. Ido, S. Guerrero, C. Mudry, and J. Mesot, EPL 88, 27008 (2009).

[188] T. Pereg-Barnea, H. Weber, G. Refael, and M. Franz, Nature Physics 6, 44 (2009).

[189] Jianqiao Meng, Guodong Liu, Wentao Zhang, Lin Zhao, Haiyun Liu, Xiaowen Jia, Daixiang Mu, Shanyu Liu, Xiaoli Dong, Jun Zhang, Wei Lu, Guiling Wang, Yong Zhou, Yong Zhu, Xiaoyang Wang, Zuyan Xu, Chuangtian Chen, and X. J. Zhou, Nature 462, 335 (2009).

[190] A. D. Palczewski, Takeshi Kondo, J. S. Wen, G. Z. J. Xu, G. Gu and A. Kaminski, Phys. Rev. B 81, 104521 (2010). 
[191] Takeshi Kondo, Yoichiro Hamaya, Ari D. Palczewski, Tsunehiro Takeuchi, J. S. Wen, Z. J. Xu, Genda Gu, Jörg Schmalian, and and Adam Kaminski, arXiv:1005.5309v1 (2010)

[192] Takeshi Kondo, Tsunehiro Takeuchi, Adam Kaminski, Syunsuke Tsuda, and Shik Shin, Phys. Rev. Lett. 98, 267004 (2007).

[193] Takeshi Kondo, Rustem Khasanov, Tsunehiro Takeuchi, Jörg Schmalian, and Adam Kaminski, Nature 457, 296 (2009).

[194] Y. Kohsaka, C. Taylor, P. Wahl, A. Schmidt, Jhinhwan Lee, K. Fujita, J. W. Alldredge, K. McElroy, Jinho Lee, H. Eisaki, S. Uchida, D.-H. Lee and J. C. Davis, Nature 454, 1072 (2008). 


\section{CHAPTER 8. CONCLUSIONS}

The motivation of this dissertation was to advance the field of cuprate superconductors, specifically by probing the electronic structure with ARPES. The three studies contained in this dissertation looked at different aspects of the electronic structure including FS topology, surface doping stability, and particle-hole symmetry. The first study (Chapter 5) probed the band structure of single layer cuprates. In general the superconducting transition temperature increases with the number of $\mathrm{Cu}-\mathrm{O}$ planes to a maximum of three, with single layer cuprates having the smallest $T_{c}$. From the previous sentence one would expect that all single layer cuprates should have approximately the same $T_{c}$, yet this is not the case. The study found $T_{c}$ can be related to slight changes in the FS, that is, for a given $T_{c}$ the FS should be the same. The shape of the band in Tl2201 $\left(T_{c} \sim 90 \mathrm{~K}\right)$ is almost identical to the band shape of Bi2212 $\left(T_{c} \sim 95 \mathrm{~K}\right)$. The study did not differentiate between the band shape close to the anti-node or the node, but the parallel part close to the antinode seemed to be the most important at the time of publication. In addition other possible scattering bands appear in the lower $T_{c} \operatorname{Bi2201}$ where the FS contain shadows bands while Tl2201 FS did not.

The second study (Chapter 6) set out to address some of the experimental factors which affect ARPES measurements, in particular how the partial pressure of active gases can change the band structure in-situ after cleaving. These "aging" effects have been known for some time, yet they have not been explored in great detail. First, we recreated the usual aging effects where free oxygen in the vacuum interacts with the surface, thereby overdoping/aging the sample. In addition, we found that the large amounts of $\mathrm{CO} / \mathrm{CO}_{2}$ can cause the same effects where UV light (required for ARPES) dissociates the $\mathrm{CO} / \mathrm{CO}_{2}$ into carbon and oxygen. Finally, after creating an UHV environment where contaminates are kept at exceptionally low 
levels we found a new aging effect above $200 \mathrm{~K}$. In this state the surface loses oxygen, thereby underdoping the sample which is in the opposite doping direction of aging. These two effects open the possibility for doping studies on a single sample across the phase diagram, without the inherent complication of comparing data from multiple samples and cleaves.

One of the most fervent debates in the world of cuprates is the origins of the pseudogap and how it relates to superconductivity. The field is moving towards a consensus for a competing order states where the pseudogap competes with superconductivity but the pseudogap's origins are still unknown. Knowing the exact particle-hole symmetry of the pseudogap could reveal how the pseudogap forms and what its origins might be. Towards this effort, the third study (Chapter 7) probed the symmetry of the pseudogap by looking at the thermally excited states above $E_{F}$. The original goal of the study was to recreate particle-hole symmetry breaking seen by other groups at the end of the arc. The study found no signature of particle-hole symmetry breaking at any location of the FS. The symmetric gap structure opening only near the antinode is indicative of a CDW origin of the pseudogap, similar to STM checkerboard pattern. In some theories the CDW should produce particle-hole symmetry breaking which we do not see. Since only some aspects of a CDW fit the data it is possible that another yet unknown origin of the pseudogap still exists. 